\title{
Improving the quality and cost-effectiveness of breast cancer follow-up
}

Citation for published version (APA):

Kimman, M. L. (2010). Improving the quality and cost-effectiveness of breast cancer follow-up. [Doctoral Thesis, Maastricht University]. Maastricht University. https://doi.org/10.26481/dis.20101105mk

Document status and date:

Published: 01/01/2010

DOI:

10.26481/dis.20101105mk

Document Version:

Publisher's PDF, also known as Version of record

\section{Please check the document version of this publication:}

- A submitted manuscript is the version of the article upon submission and before peer-review. There can be important differences between the submitted version and the official published version of record.

People interested in the research are advised to contact the author for the final version of the publication, or visit the DOI to the publisher's website.

- The final author version and the galley proof are versions of the publication after peer review.

- The final published version features the final layout of the paper including the volume, issue and page numbers.

Link to publication

\footnotetext{
General rights rights.

- You may freely distribute the URL identifying the publication in the public portal. please follow below link for the End User Agreement:

www.umlib.nl/taverne-license

Take down policy

If you believe that this document breaches copyright please contact us at:

repository@maastrichtuniversity.nl

providing details and we will investigate your claim.
}

Copyright and moral rights for the publications made accessible in the public portal are retained by the authors and/or other copyright owners and it is a condition of accessing publications that users recognise and abide by the legal requirements associated with these

- Users may download and print one copy of any publication from the public portal for the purpose of private study or research.

- You may not further distribute the material or use it for any profit-making activity or commercial gain

If the publication is distributed under the terms of Article $25 \mathrm{fa}$ of the Dutch Copyright Act, indicated by the "Taverne" license above, 


\section{Improving the Quality and Cost-effectiveness of \\ Breast Cancer \\ Follow-up}


COPYRIGHT @ 2010 MEREL KIMMAN, MAASTRICHT

ISBN: 978-94-6108-078-3

PRINTED BY: GILDEPRINT DRUKKERIJEN

BOOK DESIGN: REONALD WESTERDIJK 


\section{Improving the Quality and Cost-effectiveness of Breast Cancer Follow-up}

DISSERTATION

TO OBTAIN THE DEGREE OF DOCTOR

AT THE MAASTRICHT UNIVERSITY,

ON THE AUTHORITY OF THE RECTOR MAGNIFICUS, PROF. MR. G.P.M.F. MOLS, IN ACCORDANCE WITH THE DECISION OF THE BOARD OF DEANS, TO BE DEFENDED IN PUBLIC, ON FRIDAY 5 NOVEMBER 2010 AT 14.00 HOURS

BY

MEREL LOUISE KIMMAN 


\section{Supervisor}

Prof. dr. P. Lambin

\section{Co-supervisors}

Dr. L.J. Boersma

Dr. C.D. Dirksen

\section{Assessment Committee}

Prof. dr. M.F. von Meyenfeldt (Chairman)

Prof. dr. C.C.E. Koning (Amsterdam Medical Center)

Prof. dr. J.L. Severens

Prof. dr. A.M. Stiggelbout (Leiden University Medical Center)

Prof. dr. V.C.G. Tjan-Heijnen

Financial support by ZonMW - The Netherlands Organization for Health Research and Development - for the studies described in this thesis is gratefully acknowledged (project number 945-04-512).

Financial support for publication and distribution of this thesis was kindly provided by MAASTRO Clinic. 


\section{Contents}

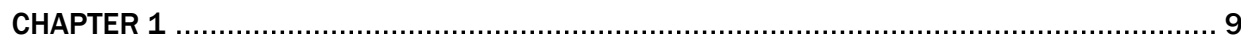

General introduction

CHAPTER 2

Follow-up after treatment for breast cancer: Why do we still adhere to frequent outpatient clinic visits?

\section{CHAPTER 3}

Improving the quality and efficiency of follow-up after curative treatment

for breast cancer - rationale and study design of the MaCare trial

CHAPTER 4

Nurse-led telephone follow-up and an educational group program

after breast cancer treatment: results of a $2 \times 2$ randomized clinical trial

CHAPTER 5

Responsiveness of the EQ-5D in breast cancer patients in their first year

after treatment

CHAPTER 6

Economic evaluation of four follow-up strategies after curative treatment for breast cancer: results of an RCT

CHAPTER 7

Patient satisfaction with nurse-led telephone follow-up after curative treatment for breast cancer

CHAPTER 8 89

Follow-up after treatment for breast cancer: One strategy fits all?

An investigation of patient preferences using a discrete choice experiment

CHAPTER 9

General discussion

REFERENCES

SUMMARY 

"Far better it is to dare mighty things - even though checkered by failure - than to dwell in that perpetual twilight that knows not victory, nor defeat." 


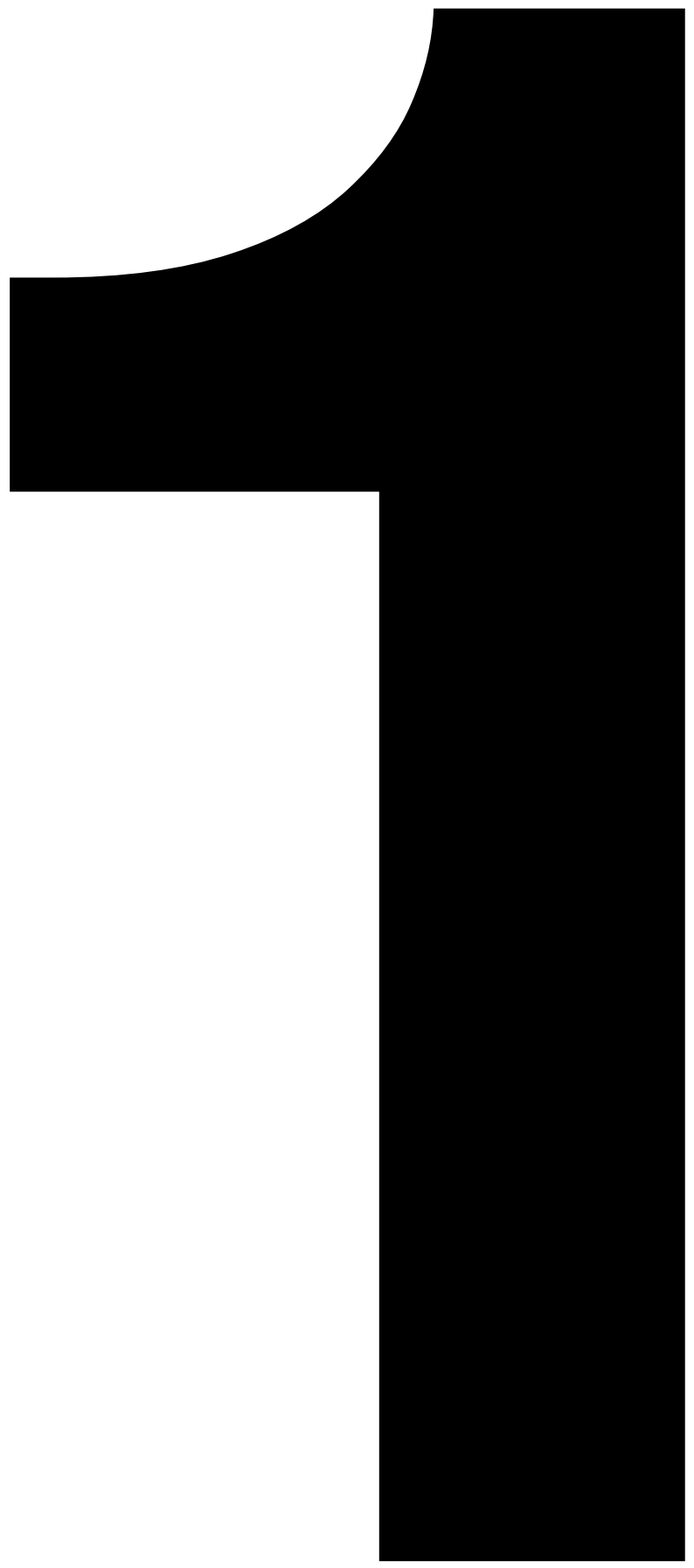

4

In the

Netherlands,

one in eight

women will be

diagnosed

with

breast cancer" 


\section{General introduction}

\section{BACKGROUND}

With an estimated 1.15 million new cases worldwide each year and a relatively good prognosis, breast cancer is the most prevalent cancer in the world today (Parkin et al., 2005). In the Netherlands, one out of eight women will be diagnosed with breast cancer at some moment in her life (Kiemeney et al., 2008). Most women with breast cancer will undergo some type of surgery in combination with radiation therapy, chemotherapy, or both.

While considerable research and attention has focused on the time of diagnosis, treatment and survivorship, little attention has been paid to the period immediately following the completion of treatment. In this first year after treatment, but also thereafter, women may experience physical, emotional and social problems (Ganz et al., 1996). Common concerns include fear of recurrence, uncertainty about the future, and coming to terms with changes in body image and life roles (Renton et al., 2002; Wyatt et al., 1993). Physical symptoms are another concern for breast cancer survivors. Fatigue, recurrent pain and sensory discomfort in the surgical area, lymph edema, and hormonal changes that can cause menopausal symptoms, are among the most troubling symptoms following treatment. 
In most countries, women are asked to come for frequent follow-up visits to the hospital following their completion of treatment, especially in the first year (Pestalozzi \& Castiglione, 2008). The main objectives of these follow-up visits are the early detection of a loco regional recurrence or a second primary breast cancer, the detection and registration of side effects of treatment and provision of information and psychological support (Pestalozzi \& Castiglione, 2008; Struikmans et al., 2008). However, there has been much debate whether these objectives of breast cancer follow-up are adequately met in current clinical practice (Kimman et al., 2007a; Montgomery et al., 2007; Pennery \& Mallet, 2000). First, neither the frequency (Jacobs et al., 2001; Schapira, 1993) nor the intensity (GIVIO, 1994; Rosselli Del Turco et al., 1994) of follow-up seems to influence the chances of cure. Second, there is a general feeling among clinicians of limited patient time during clinic visit to adequately address often complex psychosocial issues. Indeed, patients often feel uncomfortable expressing emotional concerns and asking questions (Pennery \& Mallet, 2000). Also, it has been demonstrated that outpatient clinic visits may induce anxiety because of the risk of detecting a recurrence (Allen, 2002).

Hence, not only do the current frequent follow-up strategies seem to miss their most important goals, they also depend heavily on expensive and scarce specialized knowledge for routine history taking and physical examinations. As a result, alternative follow-up strategies have been proposed, such as follow-up by a general practitioner (Grunfeld et al., 2006) and nurse-led (Koinberg et al., 2004) or telephone follow-up (Beaver et al., 2009b; Montgomery et al., 2008a). Although these studies show that different follow-up models can be equally effective as traditional hospital follow-up, measured by a variety of outcomes, these models are not yet widely applied in clinical practice. Several authors urge new research to evaluate the costs in relation to the benefits of current and alternative follow-up strategies (Grunfeld, 2009; Meropol et al., 2009; Montgomery et al., 2007). Furthermore, the new NICE guidelines for treatment and follow-up of breast cancer (2009) promote the incorporation of patient preferences into decision making (Harnett et al., 2009).

\section{ECONOMIC EVALUATION IN HEALTH CARE}

Economic evaluation assesses the relative 'value for money' of health interventions (Weinstein \& Stason, 1977). Health interventions are to be interpreted in a broad sense. They include preventive health programs, curative health care, rehabilitation services and palliative care, and within these health interventions, use of drugs, medical devices, behavioral therapy et cetera (Drummond et al., 2005; Gold et al., 1996). Economic evaluations provide information on the costs and consequences of health care interventions. This information may help policy makers in deciding how to allocate health care resources, provide evidence for clinical guidelines and promote the transparency of health care. 
There are several types of economic evaluations that differ in the way in which the consequences of health care interventions are taken into account (Drummond et al., 2005). In a cost-minimization analysis (CMA) only the costs of the alternative interventions are compared. A CMA is performed if the alternatives are equally effective, but its use has been criticized as it is rarely an appropriate method of analysis (Briggs \& O'Brien, 2001). In a cost-benefit analysis (CBA) consequences are expressed in monetary terms. $\mathrm{CBA}$ is not frequently used though, as assigning monetary values to health outcomes is difficult and controversial (Anell \& Norinder, 2000). In a cost-effectiveness analysis (CEA) consequences are measured in natural or physical units, e.g. detected cases of breast cancer, prevented strokes, or life years gained. Cost-utility analysis (CUA) is a specific form of CEA, in which the effects of health care interventions on life expectancy and healthrelated quality of life (HRQoL) are combined (Drummond et al., 2005; Gold et al., 1996). A widely used approach which combines outcomes is the Quality-Adjusted Life Year (QALY); it assigns to each period of time a value corresponding to the HRQoL during that period. The value (i.e. utility) typically lies on a scale between 0 (to represent death) and 1 (to represent full health). The number of QALYs related to a health outcome is then expressed as the value given to a particular health state multiplied by the length of time spent in that health state (Gold et al., 1996).

In CEA the economic value of two alternative interventions is compared by dividing the difference in costs between the alternatives by the difference in health outcomes. If QALYS are the measure of outcome, health care interventions are compared in terms of their incremental cost per QALY. Thus, the aim of CEA is not just to minimize costs, but also to provide information on the value-for-money or so-called incremental cost-effectiveness, that is the additional costs needed to obtain additional health gains (Drummond et al., 2005; Gold et al., 1996). The decision about which intervention to provide requires information about the maximum amount of Euros that funders (e.g. society, insurance companies) are willing to pay for these additional health gains.

\section{THE PATIENT PERSPECTIVE}

Policy makers and clinicians increasingly aim to take the patient's perspective into account in medical decision making (Coulter, 1997; Harnett et al., 2009). Patients may respond better to treatment and comply better to guidelines when they are involved in decision making and satisfied with their care and treatment setting (Jackson et al., 2001; Kizer, 2002; Worthington, 2004). Patient satisfaction is believed to reflect personal preferences of the patient, the patient's expectations, and the realities of the care received (Ware et al., 1983). Hence, satisfaction and preferences are not only related to health gains, but also to benefits beyond health gains, such as the provision of information, location of treatment and staff attitudes. Patient satisfaction can be measured using validated questionnaires (Hagedoorn et al., 2003).

Another method which is increasingly used in health care to measure satisfaction and 
preferences is the discrete choice experiment (DCE) (Ryan, 1999b; Ryan \& Gerard, 2003).

A DCE is an attribute based stated preference valuation technique which is grounded in economic theory (Lancaster, 1966; McFadden, 1974) and enables consideration of the contribution to overall value of each of the defined characteristics or attributes of an intervention (Louviere et al., 2000). Preferences are quantified by analyzing the responses that respondents provide in surveys about how they would behave in hypothetical choice situations. Respondents are asked to choose their preferred alternative from a set of two or more hypothetical alternatives. The alternatives are health care interventions (e.g. follow-up strategy) described by their key characteristics (attributes), such as the health care provider and frequency of visits. The respondents' evaluation of an alternative depends on the levels of the attributes (for example, a nurse, medical specialist or general practitioner). The relative importance of attributes and levels to respondents and the trade-offs made between them, can be assessed by asking respondents to make choices between multiple alternatives with different levels of attributes. DCEs are found to be a valid and reliable approach to elicit preferences in a health care context (Bryan et al., 2000) and are recognized as a useful tool for medical decision making (Lancsar \& Louviere, 2008; Ryan \& Gerard, 2003).

\section{OBJECTIVE OF THE THESIS}

The objective of this thesis is threefold. First, the effectiveness of nurse-led telephone follow-up instead of hospital follow-up and of a short educational group program (EGP) in addition to follow-up are investigated in a randomized controlled setting. The primary measure of effectiveness is patient-reported health-related quality of life (HRQoL). Furthermore, feelings of anxiety and control and other psychological measures are investigated. Second, an economic evaluation from the societal perspective is performed to determine the most cost-effective out of four follow-up strategies (i.e. hospital follow-up, nurse-led telephone follow-up and both strategies combined with the EGP). Third, the patient's perspective on breast cancer follow-up is further explored. Patient satisfaction with nurse-led telephone follow-up is compared to satisfaction with hospital follow-up. Moreover, the strength and heterogeneity of patient preferences for specific characteristics of follow-up are explored using discrete choice experimentation. Research results are thought to improve resource-allocation decisions and enhance dissemination of cost-effective follow-up strategies.

\section{OUTLINE OF THESIS}

CHAPTER 2 outlines a literature review on current follow-up strategies and their effectiveness in terms of detecting recurrences and providing information and support. Implications for future research are discussed, taking into account existing knowledge 
on patients' needs and expectations. CHAPTER 3 provides a detailed description of the interventions, design and execution of the study protocol of the randomized controlled trial (RCT). The RCT with a $2 \times 2$ factorial design investigates the effectiveness of nurse-led telephone follow-up and EGP in the first year after breast cancer treatment. In CHAPTER 4 the RCT results are presented. The effectiveness of nurse-led telephone follow-up and EGP are measured by HRQoL, feelings of anxiety and control, and role functioning and emotional functioning. In CHAPTER 5 results are reported of a study investigating the responsiveness of the EQ-5D in breast cancer patients after treatment. In economic evaluations, the EQ$5 D$ is often used to measure utilities for the purpose of QALY calculations. It is investigated whether changes in EQ-5D utility scores, which are determined using societal values for health states, are representative for patient reported health changes. CHAPTER 6 describes results of the economic evaluation performed alongside the RCT. Four followup strategies for the first year after treatment are compared. Following guidelines for economic evaluations a societal perspective is taken, including all relevant costs and effectiveness expressed in QALYs. CHAPTER 7 deals with patient satisfaction with nurseled telephone follow-up regarding technical competences of the health care provider, interpersonal aspects, access of care and general satisfaction. CHAPTER 8 describes results of the discrete choice experiment used to explore patient preferences for several characteristics of breast cancer follow-up. CHAPTER 9 summarizes the main research findings of this thesis and offers a general discussion, addressing some considerations related to the research and several recommendations for clinical practice and policy and future research. 


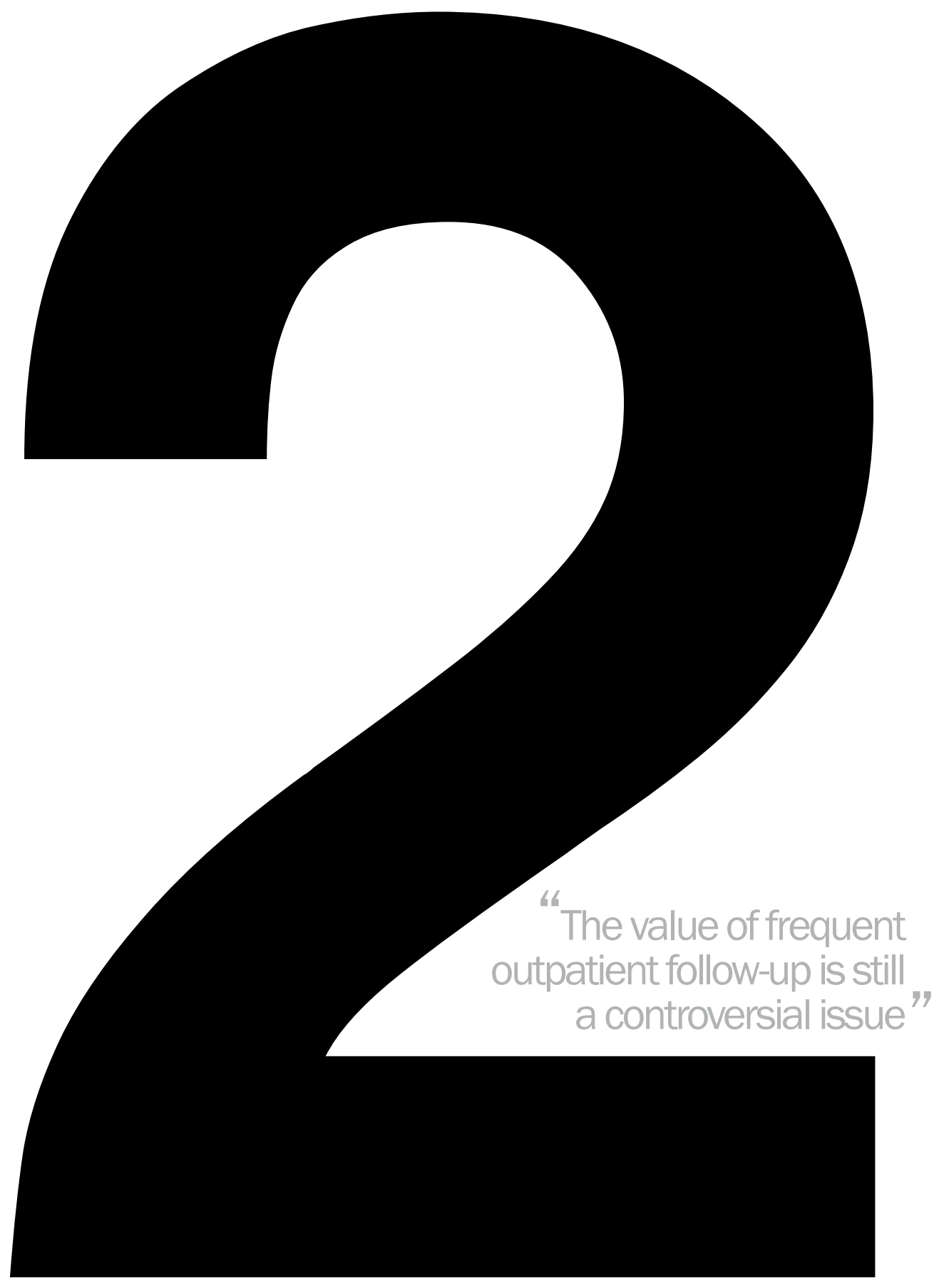




\section{Follow-up after treatment for breast cancer: why do we still adhere to frequent outpatient clinic visits?}

KIMMAN ML, VOOGD AC, DIRKSEN CD, FALGER P, HUPPERETS P, KEYMEULEN K, HEBLY M, DEHING C, LAMBIN Ph, BOERSMA L.

EUR J CANCER 2007;43(4):647-53

\section{ABSTRACT}

Follow-up after curative treatment for breast cancer consists of frequent outpatient clinic visits, scheduled at regular intervals. Its aim is primarily to detect local disease recurrence, or a second primary breast cancer, but also to provide information and psychosocial support. The cost-effectiveness of these frequent visits is being questioned however, leading to a search for less intensive follow-up strategies, such as follow-up by the general practitioner, patient-initiated or nurse-led follow-up or contact by telephone. These strategies are generally considered to be safe, but they are not yet widely accepted in clinical practice. Since brief interventions based on self-education and information have been shown to be able to improve quality of life, we hypothesize that these interventions may lead to a better acceptance of reduced follow-up by both patients and professionals. 


\section{INTRODUCTION}

After curative treatment for breast cancer women frequently attend scheduled follow-up examinations. The main objective of these examinations is to detect local disease recurrence or a second primary breast cancer in an early stage, hoping that this may increase the chances of cure. Yet, follow-up should also provide information and psychological support. Another aim is to collect data on late effects of surgery, radiotherapy and chemotherapy for audit or research and to provide feedback to physicians (Rojas et al., 2005; Rutgers et al., 1989). However, there has been much debate whether these objectives of breast cancer follow-up are adequately met in current clinical practice (Brada, 1995; Dewar, 1995). First, neither the frequency (Jacobs et al., 2001; Schapira, 1993) nor the intensity (GIVIO, 1994; Rosselli Del Turco et al., 1994) of follow-up have been shown to influence the chances of cure. Second, there is a general feeling amongst clinicians that there is limited time during the outpatient clinic visit to adequately address often complex psychosocial issues. Indeed, patients often feel uncomfortable with expressing emotional concerns and asking questions (Pennery \& Mallet, 2000). Also, it has been demonstrated that the outpatient clinic visits may induce anxiety because of the risk of detecting tumor relapse (Allen, 2002). Finally, to provide feedback to professionals on the effectiveness and side-effects of their treatments, less frequent follow-up may be sufficient as well. Not only do the current frequent follow-up strategies seem to miss their most important goals, they also depend heavily on expensive and scarce specialized knowledge for routine history taking and physical examinations. Financial constraints force oncologists and policy makers to search for alternative, more cost-effective, follow-up strategies. The aim of this paper is to explore the literature for scientific evidence why physicians and patients should still adhere to frequent outpatient clinic visits after breast cancer treatment, as recommended in the current European and American guidelines for breast cancer follow-up. In addition, we summarize studies on alternative strategies, focusing on the two main goals of breast cancer follow-up: 1) detecting recurrences or new primaries and 2) providing psychosocial support to improve quality of life (QoL). Finally, implications for future research are discussed, taking into account existing knowledge on patients' needs and expectations.

\section{CURRENT FOLLOW-UP STRATEGIES AND THEIR EFFECTIVENESS}

In Europe, the European Society for Medical Oncology (ESMO) recommends that follow-up of primary breast cancer consists of history taking and physical examinations every three to six months in the first three years after treatment, every six to 12 months for the next three years, and annually thereafter. A mammography is taken every one to two years. More intensive surveillance (i.e. with additional radiological examinations of liver, lungs and bones and laboratory tests) is not routinely recommended for asymptomatic patients (ESMO, 2001). In a recent update of the follow-up guidelines by the American Society of Clinical Oncology (ASCO) a similar frequency of history taking, physical examination 
and mammography was proposed. However, there is no high-level evidence supporting these frequent follow-up visits and the current practice of mammography surveillance (Khatcheressian et al., 2006).

\section{Effectiveness of follow-up on detecting recurrent disease}

Intensive surveillance used to be common practice in the seventies and eighties. Large randomized trials by Roselli del Turco et al. (1994) and the GIVIO investigators (1994) have shown that follow-up based on routine outpatient clinic visits combined with annual mammography is as effective with regard to overall survival, as follow-up with intensive surveillance. Since then, several papers with varying levels of evidence have been published questioning even the effectiveness of routine outpatient clinic visits (de Bock et al., 2004a; Jacobs et al., 2001; te Boekhorst et al., 2001; Wheeler et al., 1999). Wheeler et al. (1999) performed a longitudinal study of 416 consecutive patients after the diagnosis of primary breast cancer and found that frequent early follow-up provided no clear clinical gain for the great majority of patients, since early relapse was rare in the first year. Te Boekhorst et al. (2001) conducted a retrospective review in 270 patients with recurrent breast cancer and also found the clinical impact of follow-up to be low, as most patients had symptomatic recurrences (63\%). If specifically looking at loco regional recurrences, routine follow-up was more effective, detecting $66 \%$ of these recurrences. However, early detection did not translate into improved survival.

Stronger evidence is available from a meta-analysis of twelve studies by de Bock et al. (2004a). This analysis included 5045 breast cancer patients and 378 isolated loco regional recurrences and showed that approximately $40 \%$ of recurrences were diagnosed in asymptomatic patients during routine visits or routine tests $(95 \%$ confidence interval $35 \%$ to $45 \%$ ). Forty-one percent of recurrences were diagnosed outside these routine visits and tests and 18\% of recurrences were diagnosed in symptomatic patients at their routine visits. Although the rate of women diagnosed during routine follow-up with an asymptomatic recurrence seems significant, the overall incidence of loco regional recurrence is low. Thus by using frequent routine follow-up in this first two years, much effort is needed to detect only a very small proportion of curable locoregional recurrences early (de Bock et al., 2004a).

A simulation study by Jacobs et al. (2001) confirmed these findings. They tested various follow-up strategies with regard to the frequency of outpatient clinic visits. It was found that the gain in life expectancy with standard follow-up compared to no follow-up examination at all is only about 2 months in breast cancer patients aged 50 years and treated with curative intent. In older women, the gain was even less.

\section{Effectiveness of follow-up in providing psychosocial support}

Providing psychosocial support to improve QoL is another important aim of the followup. Breast cancer has an enormous psychological impact on patients and their partners, triggering fears of prolonged suffering, disability and a foreshortened life perspective. 
High levels of anxiety, depression, and distress are estimated to occur in about 35\% of patients after treatment (Clark et al., 2003). These patients should be identified and referred for specialized psychosocial support. Others depend on the follow-up visits to the medical specialist for information and some form of psychosocial support. The question remains whether short routine outpatient clinic visits are sufficient and appropriate enough for this type of support. Several studies have indicated that they are not (Allen, 2002; Pennery \& Mallet, 2000). Pennery and Mallet (2000) conducted a cross-sectional survey among 24 breast cancer patients of different age and found that most patients felt hurried and uncomfortable with expressing emotional concerns or asking questions during the outpatient clinic visit. Eighteen women stated that they would prefer to receive all or part of their follow-up from a breast care nurse. Allen interviewed six breast cancer patients and found that the scheduled clinic visits produced anxiety about breast cancer recurrence in women who were otherwise free from this particular form of anxiety. Thus, anxiety created the need to attend the clinic in order to gain reassurance about well-being (Allen, 2002). Since the numbers of patients in these studies were rather small, more research investigating the quality of psychosocial support during follow-up is urgently needed.

\section{WHAT DO PATIENTS NEED AND EXPECT?}

Several studies investigated breast cancer patients' needs after treatment and their expectations about follow-up examinations, by using interviews or questionnaires. Important needs that patients expressed were reassurance and social support (Pennery \& Mallet, 2000), confirmation, continuity, accessibility and information (Koinberg et al., 2001), cancer expertise of clinical staff and a good relationship with the doctor (AdewuyiDalton et al., 1998). Renton et al. (2002) surveyed the opinion of 134 women attending a breast cancer follow-up clinic to establish what they considered the most important topics for discussion at follow-up. Information on risk of recurrence (70\%), benefits and side effects of treatment (61\%) and the risk of breast cancer affecting their daughters (41\%) were highlighted the most. A cross-sectional survey by de Bock et al. (2004b) showed that patients' expectations of routine follow-up were high and sometimes unrealistic. Seventyfour out of 84 patients (88\%) believed that early detection of distant metastases would contribute to a cure. Therefore, most of these patients preferred additional investigations to be part of the follow-up and more than half preferred lifetime follow-up, performed by a medical specialist.

These studies show that women differ greatly in their needs and preferences regarding follow-up. Moreover, the needs and preferences deviate from available evidence on the effectiveness of routine follow-up, stressing the need for more information and education about the particular aims and effects of follow-up. 


\section{ALTERNATIVE FOLLOW-UP STRATEGIES FOR BREAST CANCER TO REDUCE CLINICAL WORKLOAD AND COSTS}

To make more efficient use of scarce specialized expertise, reduced follow-up strategies have been proposed. These include follow-up by the general practitioner or nurse, and patient-initiated follow-up (see table 1a). Also, replacing clinic visits by telephone interviews is increasingly being used as a presumably safe and cost-effective way of follow-up.

Table 1a - Trials investigating strategies for breast cancer follow-up aimed at reducing clinical workload and costs.

\begin{tabular}{|c|c|c|c|}
\hline Author (year) & Aim of the study & $\begin{array}{l}\text { Design/ } \\
\text { Participants }\end{array}$ & Results \\
\hline $\begin{array}{l}\text { Grunfeld et al } \\
(1996) \\
(1999)\end{array}$ & $\begin{array}{l}\text { To assess the effect } \\
\text { of transferring follow- } \\
\text { up from hospital to } \\
\text { general practice (GP) }\end{array}$ & $\begin{array}{l}\text { RCT: } 296 \\
\text { women with } \\
\text { stage I, II or III } \\
\text { breast cancer }\end{array}$ & $\begin{array}{l}\text { GP follow-up was not associated with in- } \\
\text { crease in time to diagnosis of recurrence } \\
\text { or decreased quality of life (QoL). No } \\
\text { significant difference between groups in } \\
\text { anxiety or depression scores was found. } \\
\text { The GP group indicated greater satisfac- } \\
\text { tion than the hospital group. }\end{array}$ \\
\hline $\begin{array}{l}\text { Grunfeld et al } \\
(2006)\end{array}$ & $\begin{array}{l}\text { To assess whether } \\
\text { GP follow-up is a safe } \\
\text { and acceptable alter- } \\
\text { native to specialist } \\
\text { follow-up }\end{array}$ & $\begin{array}{l}\text { RCT: } 968 \\
\text { women with } \\
\text { stage I, II or III } \\
\text { breast cancer }\end{array}$ & $\begin{array}{l}\text { No differences in recurrences, recur- } \\
\text { rence-related serious clinical events or } \\
\text { health related QoL were found between } \\
\text { the GP group and the specialist group. }\end{array}$ \\
\hline $\begin{array}{l}\text { Gulliford et al } \\
\text { (1997) }\end{array}$ & $\begin{array}{l}\text { To compare regular } \\
\text { follow-up with follow- } \\
\text { up restricted to the } \\
\text { time of mammog- } \\
\text { raphy }\end{array}$ & $\begin{array}{l}\text { RCT: } 211 \\
\text { women with } \\
\text { stage I, II or III } \\
\text { breast cancer }\end{array}$ & $\begin{array}{l}\text { Patients undergoing less frequent } \\
\text { follow-up did not increase their use of } \\
\text { GP or telephone services. Patients were } \\
\text { highly supportive to pursue less frequent } \\
\text { follow-up. }\end{array}$ \\
\hline $\begin{array}{l}\text { Brown } \\
\text { et al } \\
(2002)\end{array}$ & $\begin{array}{l}\text { To assess the benefits } \\
\text { of clinic follow-up } \\
\text { compared to patient } \\
\text { initiated follow-up }\end{array}$ & $\begin{array}{l}\text { RCT: } 61 \\
\text { women with } \\
\text { stage I breast } \\
\text { cancer }\end{array}$ & $\begin{array}{l}\text { No differences in QoL, psychological mor- } \\
\text { bidity or satisfaction with care were found } \\
\text { between the groups. }\end{array}$ \\
\hline $\begin{array}{l}\text { Koinberg et al } \\
(2004)\end{array}$ & $\begin{array}{l}\text { To compare follow-up } \\
\text { by the physician with } \\
\text { nurse-led follow-up on } \\
\text { demand }\end{array}$ & $\begin{array}{l}\text { RCT: } 264 \\
\text { women with } \\
\text { stage I and II } \\
\text { breast cancer }\end{array}$ & $\begin{array}{l}\text { No statistically significant differences in } \\
\text { levels of anxiety, depression and satisfac- } \\
\text { tion were found between the groups. } \\
\text { Also, no differences concerning time to } \\
\text { recurrence or death were detected. }\end{array}$ \\
\hline
\end{tabular}

\section{Follow-up by the general practitioner}

In a randomized study by Grunfeld et al. (1996), 296 women with stage $\leq$ III breast cancer received routine follow-up either in the hospital or in general practice. Results indicated that general practice follow-up was not associated with increase in time to diagnosis of recurrence, increase in anxiety, or deterioration in health related QoL, and patients' 
satisfaction with follow-up was higher, compared to hospital follow-up (Grunfeld et al., 1999a). The economic evaluation showed that the mean costs per patient for 18 months of follow-up in general practice were only one third of those per patient in the hospital follow-up (Grunfeld et al., 1999b). A later similarly randomized trial by Grunfeld et al. (2006) with long-term follow-up in 986 patients showed that follow-up can be offered by the general practitioner without concern that important recurrence-related serious events will occur more frequently or that health-related QoL will be affected negatively compared to routine follow-up.

\section{Patient-initiated follow-up}

In a study by Gulliford et al. (1997), the experiences of breast cancer patients who attended an outpatient clinic for follow-up were compared with those in whom routine follow-up was restricted to a single visit only after mammography. All patients were advised to examine their breasts each month and to request an appointment if they felt a lump or developed any other symptom. Of 211 eligible patients, 196 (93\%) opted for randomization in the study. No increased use of local practitioner services or telephone calls to the hospital was apparent in the group randomized to less frequent follow-up. Although QoL was not analyzed in this study, this cohort of patients was highly supportive of the option to pursue less frequent follow-up. Brown et al. (2002) compared routine outpatient clinic follow-up and patient-initiated follow-up in women treated for stage I breast cancer in a small randomized trial $(n=62)$, and found that patient-initiated follow-up was a safe and possibly cost-effective alternative to routine follow-up for women at low risk of recurrence. However, this study was not sufficiently powered to draw firm conclusions.

\section{Nurse-led telephone follow-up}

A literature review by Cox and Wilson on nurse-led services and telephone interventions in follow-up for patients with all forms of cancer showed that nurse-led follow-up services were acceptable, appropriate and effective (Cox \& Wilson, 2003). Specifically looking at breast cancer, Koinberg et al. (2004) conducted a randomized trial comparing usual followup by the medical specialist ( $n=131$ ) with nurse-led follow-up on demand $(n=133)$. In the nurse-led group, patients met with an experienced nurse three months after surgery. They received information about how to recognize recurrence and were requested to contact the nurse for any concerns related to breast cancer. The nurse arranged a mammography at one-year intervals and reported the results by telephone or letter. No statistically significant differences were found in levels of anxiety, depression or satisfaction with followup between the two groups. In addition, there were no differences in time to recurrence or death. These data suggest equal validity of both approaches, but the limited number of patients prohibits drawing firm conclusions on this subject. Furthermore, the nurse-led group reported 450 less visits to the medical specialist but 177 more phone calls and 88 more visits to the nurse, than the specialist-led group (Koinberg et al., 2004). No costs were calculated, but it may be thought that the substantial reduction in medical specialist 
visits would have led to cost reduction. Similar results were found in trials investigating nurse-led telephone follow-up as an alternative to conventional outpatient clinic follow-up in other cancers (Helgesen et al., 2000; Sardell et al., 2000).

\section{INTERVENTIONS AFTER CANCER TREATMENT TO PROVIDE PSYCHOSOCIAL SUPPORT}

A number of studies have been performed using various group intervention formats to provide psychosocial support for cancer patients. Results varied and refusal rates for extensive programs were often high (Clark et al., 2003). Only few studies focused on women treated for breast cancer (see table 1b).

Table $1 b$ - Trials investigating group interventions for women treated for primary breast cancer.

\begin{tabular}{|c|c|c|c|}
\hline Author (year) & Aim of the study & $\begin{array}{l}\text { Design/ Partici- } \\
\text { pants }\end{array}$ & Results \\
\hline $\begin{array}{l}\text { Helgeson et al } \\
(1999) \\
(2000)\end{array}$ & $\begin{array}{l}\text { To compare the } \\
\text { effectiveness of an } \\
\text { education-based and } \\
\text { a peer-discussion } \\
\text { based group inter- } \\
\text { vention }\end{array}$ & $\begin{array}{l}\text { RCT: } 312 \text { women } \\
\text { with stage I, II or } \\
\text { III breast cancer }\end{array}$ & $\begin{array}{l}\text { The educational intervention improved } \\
\text { self-esteem and body image and re- } \\
\text { duced disturbing thoughts. Peer-assisted } \\
\text { discussion groups were only helpful for } \\
\text { women who lacked support from their } \\
\text { partners, but harmful for women with } \\
\text { high support levels. }\end{array}$ \\
\hline $\begin{array}{l}\text { Antoni } \\
\text { et al (2001) }\end{array}$ & $\begin{array}{l}\text { To test the effects } \\
\text { of a group cognitive- } \\
\text { behavioral stress } \\
\text { management inter- } \\
\text { vention }\end{array}$ & $\begin{array}{l}\text { RCT: } 100 \text { women } \\
\text { with stage } 0,1 \text {, } \\
\text { II or III breast } \\
\text { cancer }\end{array}$ & $\begin{array}{l}\text { The intervention reduced prevalence of } \\
\text { moderate depression, but did not affect } \\
\text { other measures of emotional distress. }\end{array}$ \\
\hline $\begin{array}{l}\text { Koinberg et al } \\
(2006)\end{array}$ & $\begin{array}{l}\text { To compare a } \\
\text { multidisciplinary } \\
\text { educational program } \\
\text { (MP) with traditional } \\
\text { follow-up visits to a } \\
\text { physician }\end{array}$ & $\begin{array}{l}\text { Prospective } \\
\text { non-randomized } \\
\text { study: } 96 \text { women } \\
\text { with stage I or II } \\
\text { breast cancer }\end{array}$ & $\begin{array}{l}\text { Women in the MP increased their physi- } \\
\text { cal and functional well-being. Women } \\
\text { with traditional follow-up increased } \\
\text { functional well-being, while social/family } \\
\text { well-being decreased over time. Women } \\
\text { with traditional follow-up also scored } \\
\text { lower in their overall coping capacity. }\end{array}$ \\
\hline
\end{tabular}

Helgeson et al. (1999) found in a randomized trial $(n=312)$ that an education-based intervention increased psychological and physical functioning by enhancing self-esteem and reducing disturbing intrusive thoughts about the illness, both immediately following and six months after the intervention. In addition, peer-assisted discussion groups were helpful for a subgroup of women who lacked emotional support from their partners, but could be harmful for women with high support levels (Helgeson et al., 2000). The positive effects of the education intervention dissipated with time, but the differences with the control group remained significant over a 3-year period $(n=252)$ (Helgeson et al., 2001). A randomized trial by Antoni et al. (2001) $(n=100)$ tested effects of a 10-week group cognitive- 
behavioral stress management intervention that focused on coping with daily stressors related to breast cancer and on optimizing one's use of social resources. The intervention reduced prevalence of moderate depression and enhanced optimism about the future, but did not affect other measures of emotional distress, such as mood disturbance and thought intrusion and avoidance. A recently published non-randomized study by Koinberg et al. (2006) compared a short multidisciplinary educational program $(n=50)$ with traditional follow-up visits to a physician $(n=46)$ after breast cancer surgery. The program was led by a specialized nurse in oncology and comprised four sessions of lectures and discussions on topics related to breast cancer. The program aimed to strengthen the selfcare ability of participants, in addition to promoting health and to facilitating sense of coherence. Results of this study indicated that the educational program was comparable to a traditional physician follow-up program in terms of well-being, aspects of self-care and coping ability, and could thus be an alternative to traditional physician follow-up.

\section{IMPLICATIONS FOR FUTURE RESEARCH}

In summary, the randomized clinical trials investigating the effects of less frequent followup (Brown et al., 2002; Gulliford et al., 1997) and nurse-led follow-up (Koinberg et al., 2004) were too small to allow firm conclusions on the safety of these reduced followup strategies. Nevertheless, several retrospective studies (de Bock et al., 2004a; te Boekhorst et al., 2001) and the simulation study of Jacobs et al. (2001) strongly suggest that only few patients would benefit from frequent routine follow-up visits in terms of early detection of curable loco regional recurrence. Since this early detection of loco regional recurrences does also not seem to translate into improved survival (Wheeler et al., 1999), the cost-effectiveness of frequent follow-up in terms of survival benefit is highly questionable. In addition to primarily clinical considerations, routine follow-up also has a psychosocial component, but there is only limited time during outpatient clinic visits to address this. Moreover, it has been suggested that these visits may induce a high level of anxiety (Allen, 2002).

In the last decade, alternative strategies for follow-up have been proposed. Trials investigating less frequent follow-up, nurse-led follow-up, and shared care with the patient's family doctor, show promising results with respect to QoL and patient satisfaction (Grunfeld et al., 2006; Gulliford et al., 1997; Koinberg et al., 2004). Additionally, a short educational group program may be able to reduce outpatient clinic visits to the specialist (Koinberg et al., 2006). Yet, alternative follow-up strategies are not widely applied in clinical practice and frequent conventional oncologist follow-up is still common practice. Several reasons could explain this situation. First, financial incentives for medical specialists might play a role. A more likely explanation could be the, probably false, perception of many medical specialists that patients need frequent reassurance. In addition, many patients have false expectations that frequent follow-up visits will result in better overall 
survival (de Bock et al., 2004b). Finally, another reason may be that trial-based economic evaluations, supporting the conclusions that reduced strategies are indeed cost-effective to society, are scarce.

We hypothesize that improvements in psychosocial support and education of patients may lead to better acceptance rates in both patients and medical specialists with respect to a reduced follow-up strategy. Furthermore, we recognize that the cost-effectiveness of both reduced follow-up policies as well as educational group programs should be determined to justify implementation of new strategies.

In the Netherlands, we have started a randomized trial investigating the cost-effectiveness of four different follow-up strategies for primary breast cancer patients. These strategies include the routine frequent outpatient clinic visits as described in current guidelines; follow-up in the first two years reduced to annual mammography, with telephone interviews by a nurse practitioner at the other time points of the routine strategy $(3,6,12$ and 18 months) and; each of these strategies combined with a short educational group program, focusing on enhancing QoL. In this study, nurse practitioners have a major role in the follow-up. The increased use of nurse practitioners is an important trend in cancer care; they are uniquely educated to provide quality specialty care, are easily accessible and they can provide continuity of care to the patients. Thus, their role may lead to high patients' satisfaction. Also, they are likely to reduce costs associated with follow-up.

\section{CONCLUSION}

The value of frequent outpatient follow-up in the first few years after curative treatment for breast cancer is still a controversial issue. There is no strong evidence that routine outpatient clinic visits are effective with regard to the detection of recurrences, as most recurrences are detected between scheduled appointments. Moreover, routine followup has a major impact on costs and staff resources. Therefore, alternative follow-up strategies have been tested and well-received by patients, not adversely affecting patient satisfaction or QoL. However, there still seem to be barriers preventing the full fledged implementation of these new strategies in clinical practice. More psychosocial support and better education and information to the patient, may lead to a better acceptance of reduced follow-up policies. A trial investigating the cost-effectiveness of a reduced followup strategy, combined with additional psychosocial support and education, is currently underway. 


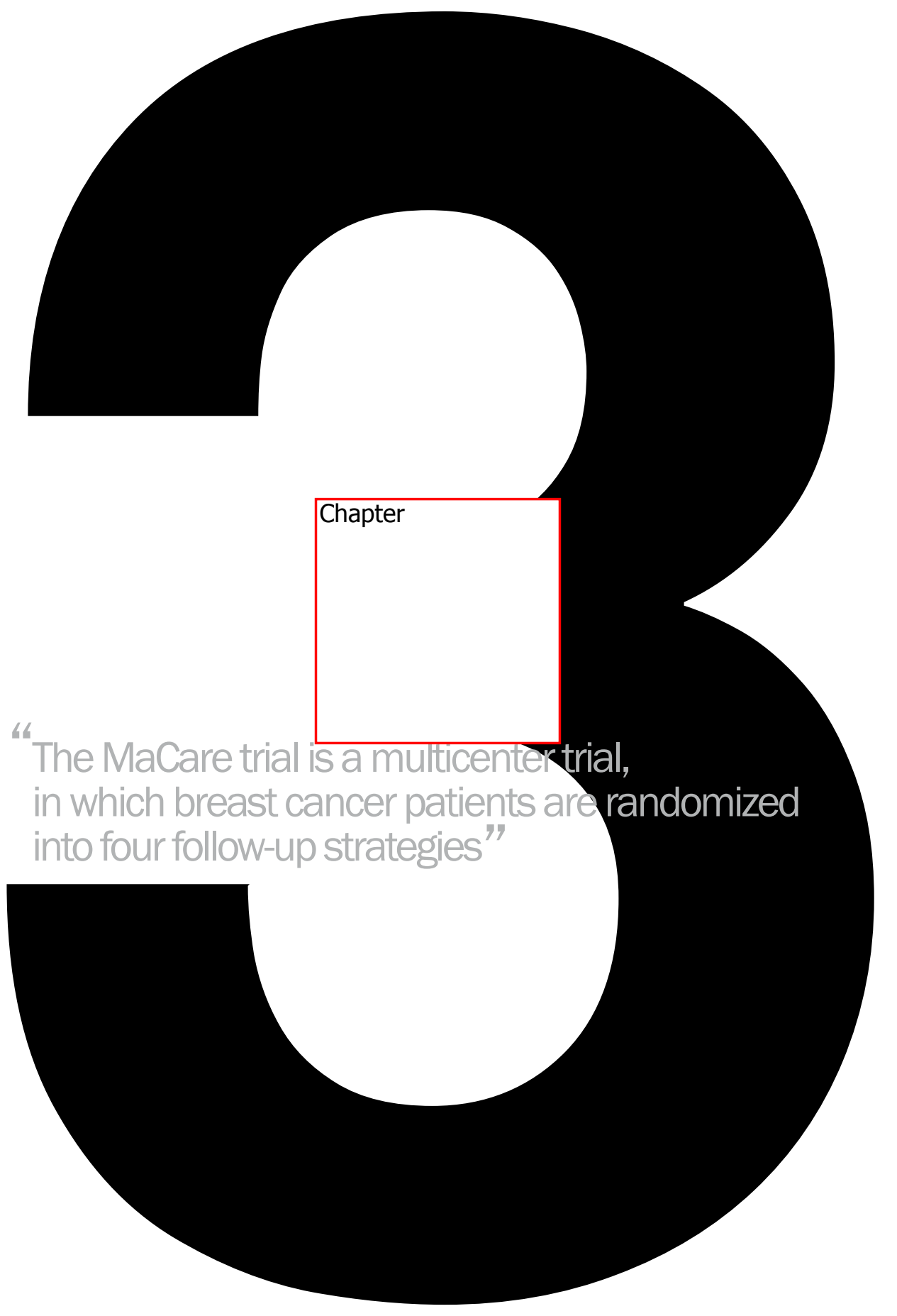




\title{
Improving the quality and efficiency of follow-up after curative treatment for breast cancer - rationale and study design of the MaCare trial
}

KIMMAN ML, VOOGD AC, DIRKSEN CD, FALGER P, HUPPERETS P, KEYMEULEN K, HEBLY M, DEHING C, LAMBIN Ph, BOERSMA L.

BMC CANCER 2007;7(1):1

\begin{abstract}
[Background] This paper describes the design of the MaCare trial. The MaCare trial compares the cost-effectiveness of four follow-up strategies for curatively treated breast cancer patients. We investigate the costs and effects of nurse-led telephone follow-up and a short educational group program. [Methods/design] The MaCare trial is a multicenter randomized clinical trial in which 320 breast cancer patients are randomized into four follow-up strategies, focused on the first 18 months after treatment: 1) standard follow-up; 2) nurse-led telephone follow-up; 3) arm 1 with the educational group program; 4) arm 2 with the educational group program. Data is collected at baseline, and 3, 6, 12 and 18 months after treatment. The primary endpoint of the trial is cancer-specific quality of life (QoL) as measured by the global health/QoL scale of the EORTC QLQ-C30. Secondary outcomes are perceived feelings of control, anxiety, patients' satisfaction with follow-up and costs. A cost-effectiveness analysis will be performed from a societal perspective. [Discussion] Reduced follow-up strategies for breast cancer have not yet been widely applied in clinical practice. Improvement of psychosocial support and information to patients could lead to a better acceptance of reduced follow-up. The MaCare trial combines a reduced follow-up strategy with additional psychosocial support. Less frequent follow-up can reduce the burden on medical specialists and costs. The educational group program can improve QoL of patients, but also less frequent follow-up can improve QoL by reducing the anxiety experienced for each follow-up visit. Results of the trial will provide knowledge on both costs and psychosocial aspects regarding follow-up and are expected in 2009.
\end{abstract}




\section{BACKGROUND}

With an estimated 1.15 million new cases worldwide each year, breast cancer is by far the most frequent cancer in women. Because of its high incidence and relatively good prognosis, breast cancer is also the most prevalent cancer in the world today (Parkin et al., 2005). After curative treatment for breast cancer women frequently attend scheduled follow-up examinations. Usually the follow-up is most frequent in the first 2-3 years (2-4 times a year); thereafter the frequency is reduced to once a year in most countries. The main objective of these examinations is to detect local disease recurrence or a second primary breast cancer in an early stage, hoping that this may increase the chances of cure. Follow-up should also provide information and psychological support. Another aim is to collect data on late effects of surgery, radiotherapy and chemotherapy for audit or research and to provide feedback to physicians (Rojas et al., 2005; Rutgers et al., 1989). However, there is much debate whether the objectives of breast cancer follow-up are adequately met in current practice (Brada, 1995; Dewar \& Kerr, 1985). Schapira (1993) reported that the early frequent outpatient clinic visits with only physical examination and an annual mammography do not improve early detection of recurrence or overall survival. Since then, several studies have investigated the effect of the frequency (Jacobs et al., 2001) and intensity (GIVIO, 1994; Rosselli Del Turco et al., 1994) of follow-up on overall survival, without finding evidence supporting the necessity of frequent or intensive followup. Furthermore, instead of providing psychosocial support, it has been demonstrated that the outpatient clinic visit may induce anxiety because of the risk of detecting tumor relapse (Allen, 2002; Pennery \& Mallet, 2000).

Although the current frequent follow-up strategies thus seem to miss their most important goals, they do depend heavily on expensive and scarce specialized knowledge for routine history taking and physical examination. Financial constraints have forced oncologists and policy makers to search for alternative and cost-effective follow-up strategies. Studies on follow-up by the general practitioner, patient-initiated or nurse-led followup or contact by telephone showed no difference in time to detection of recurrence or patients' perceived quality of life (QoL) (Brown et al., 2002; Grunfeld et al., 2006; Grunfeld et al., 1996; Gulliford et al., 1997; Koinberg et al., 2004). Nevertheless, these reduced strategies have not yet been widely accepted and applied in clinical practice. This may on the one hand be ascribed to the perception of many medical specialists that most patients need reassurance and psychosocial support, and on the other hand to the false expectations of many patients that frequent follow-up will result in a better overall survival (de Bock et al., 2004b). Improvement of the psychosocial support and education of the patients may lead to a better acceptance of both patients and medical specialists of a reduced follow-up policy. A number of studies have been performed using various group intervention formats for breast cancer patients to provide psychosocial support. However, most interventions mainly focused on intensive psychological support (10 sessions or more), showing varying results and often high refusal rates (Clark et al., 2003). 
No trials have been performed that combine a reduced follow-up policy in the first two years with an acceptable strategy to provide information and education. For this reason, two interventions for early breast cancer follow-up were developed for the study that is discussed in this paper; nurse-led telephone follow-up and a short educational group program (EGP). The primary aim of the so-called MaCare trial (Mamma carcinoma and after(are) is to investigate the impact of these interventions on cancer-specific QoL and costs and thus to determine which of these follow-up strategies is the most cost-effective. This paper reports on the methodological design of the trial as well as the contents of the interventions and the economic evaluation.

\section{METHODS}

\section{Study design}

The MaCare trial is a multicenter randomized trial, in which breast cancer patients who are treated with curative intent are randomized between four follow-up strategies (see figure 1, page 28):

1) Follow-up as usual; five outpatient clinic visits in the first 18 months (at 3, 6, 9, 12 and 18 months), with a mammography at one year.

2) Nurse-led telephone follow-up; a mammography at one year combined with an outpatient clinic visit, and telephone interviews by a breast care nurse $(\mathrm{BCN})$ or nurse practitioner (NP) at the same time points as during the usual follow-up (i.e. 3, 6, 9 and 18 months).

3) Similar to arm 1, combined with the EGP.

4) Similar to arm 2, combined with the EGP.

After 18 months patients return to the current follow-up strategy, i.e. follow-up once a year.

\section{Setting}

The trial is carried out in seven hospitals and two radiotherapy clinics located in the south of The Netherlands. Medical specialists working in the participating centers have been recruiting patients since July 2005 and will continue recruitment until approximately September 2007. Follow-up as usual takes place in the hospital where surgery and chemotherapy were performed, alternating between the surgeon, medical oncologist and radiation oncologist. The telephone follow-up is performed by the nurse practitioner (NP) or breast care nurse (BCN) working at this hospital. An NP is a registered nurse who has acquired (at masters level) the expert knowledge base, complex decisionmaking skills and clinical competencies for expanded practice. The BCN is a qualified nurse who has had specialist training in breast care and who guides the patient throughout treatment. In this paper the term BCN is used and refers to both types of nurses. The EGP is held at cancer information centers. These centers are specialized in providing support to (ex-) cancer patients and their relatives through education and organizing activities. Selecting participants from multiple centers and locations in the 
Netherlands allows us to include a representative selection of breast cancer patients. Participation is voluntary and after written informed consent is obtained. Prior to the start of the study the protocol was approved by the Medical Ethical Committee of MAASTRO Clinic. Furthermore, all participating centers signed a declaration on local feasibility before inclusion of their first patient, according to the Dutch law and regulations.

Breast cancer patients without distant metastases, within 6 weeks after treatment $(n=320)$

$\downarrow$

Surgery +/- radiotherapy +/- chemotherapy; included by last treating medical specialist

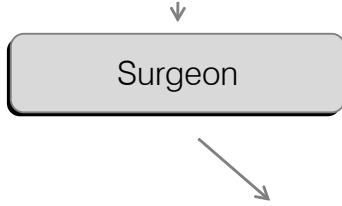

Medical oncologist

Radiation oncologist

1. Follow-up as usual

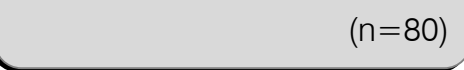

3. Follow-up as usual + EGP

$$
(n=80)
$$

Figure 1. Design of MaCare trial
2. Nurse-led telephone followup

$$
(n=80)
$$

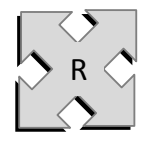

4. Nurse-led telephone followup + EGP $(n=80)$

\section{Study population}

\section{Selection of participants}

Patients are recruited and included within six weeks after completion of treatment with curative intent by the medical specialist of the last treatment modality. Eligibility criteria are summed up in table 1. If eligible, the medical specialist introduces the MaCare trial to the patient and gives her a detailed information sheet. Within two weeks, the research assistant contacts the patient to explain the aim and implications of the trial again. Eligibility criteria are checked once more and the research assistant asks whether the patient wants to participate. 
Table 1: Study inclusion and exclusion criteria

Inclusion criteria
Female patients with breast cancer without distant metastases
WHO performance scale $0-2$
chemotherapy)
Treatment completed < 6 weeks prior to randomization
Able to speak and read fluently in Dutch

\section{Exclusion criteria}

Participation in another trial, requiring more frequent follow-up

Medical diseases (treatment-related side-effects, concomitant tumors) requiring frequent follow-up

\section{Non-participants}

Patients who are eligible for inclusion but not interested in participation are asked to fill out the same baseline questionnaires (see outcome parameters) as participants. By assessing whether there are differences between participants and non-participants the external validity of the study results can be determined.

\section{Sample size calculations}

The primary outcome measure is cancer specific QoL at 12 months after randomization, measured by the global health/QoL scale of the EORTC QLQ-C30. A baseline score of 64 with a standard deviation of 17 (Gijsen \& Koppejan-Rensenbrink, 2003; Nagel et al., 2001) is considered as a starting point. In an analysis of the clinical significance of changes in QoL, Osoba et al. (1998) showed that patients judge a change between 5-10 to be small, between 10-20 to be moderate, and more than 20 to be large. Consequently, we considered a change smaller than 5 points to be no change. To show that QoL of patients in arms 2 and 4 (telephone follow-up) is at least similar to patients in arm 1 and 3 (usual follow-up) with a power of $80 \%$ and a significance level of 0.05 , the required number of patients per group is 80 . With these 320 patients an improvement in QoL, by adding the EGP compared to no EGP, of 10 points with a power of 0.95 and a significance level smaller than 0.01 can be detected. A dropout rate of $10 \%$ is accounted for in these calculations.

\section{Randomization}

After written informed consent and completion of the baseline questionnaire, randomization by minimization is performed by the Comprehensive Cancer Center Limburg. Patients are pre-stratified by treatment modalities and hospital. After randomization, the research assistant contacts the patient and informs her about the assigned study arm, thus whether she will receive standard or telephone follow-up and whether she will be invited to join the EGP. 


\section{Interventions}

\section{Usual care}

The first study arm, the standard arm, consists of follow-up as described in the Dutch guidelines. After treatment, the patient has an outpatient clinic visit with the medical specialist or BCN every three months during the first year, every six months during the second year, and thereafter once a year until at least five years. Mammography is taken once a year.

\section{Nurse-led telephone follow-up}

In study arms 2 and 4 a mammography is taken once a year, which is combined with a visit to the medical oncologist, the surgeon, $\mathrm{BCN}$ or the radiation oncologist. At the other regular follow-up times (i.e. at 3, 6, 9 and 18 months), the BCN has a telephone interview with the patient. The telephone interview is done by open discussion and a semi structured questionnaire, including screening for physical - especially loco regional - and psychosocial symptoms, and compliance to hormonal therapy. Data on the side effects of treatment are also collected during the interview. Furthermore, the BCN informs about general well being of the patient, her family-life, relationships and work reintegration. If the patient does not feel reassured an additional appointment is made for her to come to the hospital. The telephone follow-up is mainly meant to reduce the workload of medical specialists, with possible reduction of costs, but could also influence QoL as a result of a reduction of stressful visits to the outpatient clinic (Allen, 2002). To adequately perform the telephone interview, all participating BCNs attained training, specifically developed for this study. In this training nurses are informed on the most recent developments in breast cancer treatment and follow-up, develop a semi-structured questionnaire for support during the interview, and practice their telephone communication skills with a breast cancer patient.

\section{Educational group program}

In arms 3 and 4 of the study, the patient and her partner (or close friend or family member) are invited to participate in the Educational Group Program (EGP) within three months after treatment. The basic theory underlying the EGP is the transaction process theory of stress (Lazarus \& Folkman, 1984). The group-format program focuses on the first two stages of the transaction process theory of stress, the primary and secondary appraisal. The EGP therefore consists of two interactive group sessions of 2,5 hours of patient education. In the first session, which addresses the primary appraisal, a BCN provides information on possible side effects of the different treatments and issues such as prostheses, exhaustion, hereditary issues and financial consequences. Also, signs and symptoms of recurrences are discussed. A health care psychologist concentrates on psychological consequences, like anxiety, depression and changed family role patterns. The second session focuses on the second appraisal, i.e. information is provided on possible coping strategies and actions that could be undertaken when symptoms occur. 
Furthermore, information on the various health care workers and additional interventions is given. Discussions are stimulated by the speakers, addressing issues such as how to deal with anxiety, mood changes and changes in relationships. In this way, patients and their partners are stimulated to be alert to symptoms, and to enhance self-efficacy in adopting one of the proposed actions or coping strategies. It is expected that this will lead to a better QoL. During the second session a volunteer from the Dutch Breast Cancer Association presents regional activities available for participants. The EGP is completed with a relaxation exercise. After the two sessions, participants receive a small booklet encompassing the topics discussed in the program. The BCN and health care psychologist use standardized presentations, so that the EGPs given during the time of this study are as similar as possible. Furthermore, by using standard presentations, different teams can lead the EGP and the program will be easily transferable to other settings.

\section{Primary and secondary outcome measures}

\section{Primary outcome measure}

The primary domain for improvement for both interventions in the trial is cancer-specific QoL. Cancer-specific QoL is measured by the QoL scale of the European Organisation for Research and Treatment of Cancer (EORTC) QLQ-C30. The EORTC QLQ-C30 has a validated Dutch-language version available (Aaronson et al., 1993).

\section{Secondary outcome measures}

Secondary outcome measures are perceived behavioral control, anxiety, patients' satisfaction with follow-up, generic health-related quality of life and societal costs. Perceived behavioral control is measured by the Dutch version of the Mastery Scale (Pearlin \& Schooler, 1978). A question whether the patient is experiencing emotional support from partner (if applicable) is added. Anxiety is measured by the validated Dutch version of the State-Trait Anxiety Inventory (STAI) (van der Ploeg, 1980). Patients' satisfaction with followup will be assessed using the validated Patient Satisfaction Questionnaire - NL (PSQ-NL) (Hagedoorn et al., 2003). Generic health-related quality of life is measured by the EuroQol5D (EQ-5D) (EuroQoL-Group, 1990).

Societal costs are determined by the hospital information systems and prospective costdiaries. More details on the costs are provided in the economic evaluation section.

\section{Time schedule}

Patients complete questionnaires at baseline, and at 3, 6, 12 and 18 months intervals. Costs-diaries are filled out by the patients over a four week period, at the same time points. The questionnaires and cost-diaries are sent by mail. If not returned within 14 days, patients are contacted by the research assistant and are asked to continue participation.

\section{Statistical analysis}

To evaluate differences in QoL outcome between the study arms, the data will be analyzed 
using a multivariate linear regression model. In this model both the EGP factor and the telephone factor will be taken into account. In addition, variables such as age, hospital treatment, and experienced support of a partner, will be brought into the model. The procedure will be performed four times as QoL is assessed at 3, 6, 12 and 18 months. Differences in satisfaction with care, perceived behavioral control and anxiety will be analyzed in the same way. Participation at group meetings will be analyzed using logistic regression models. Differences in medical consumption will be evaluated using a multivariate linear regression model. Subgroup analyses will be performed to identify specific patient characteristics that could predict the optimal follow-up strategy for the individual patient. Data will be analyzed according to the intention-to-treat principle.

The number of recurrences is expected to be very low with a follow-up of 18 months (Bartelink et al., 2001). Statistical analysis of the difference in time-to-detection per group will therefore be limited to descriptive statistics.

\section{Economic evaluation}

Parallel to the trial an economic evaluation will be performed, comparing the costs and effects of each separate follow-up strategy, in order to determine the most cost-effective follow-up. The cost-effectiveness comparison thus includes four different strategies corresponding to the four study-arms. The cost-effectiveness ratio(s) will be expressed as the incremental cost per quality-adjusted life year (QALY). In order to be able to construct a QALY, the scores on the five dimensions of the EuroQol (Dolan, 1997) are used. The costeffectiveness analysis is performed from a societal perspective. Sensitivity analyses will subsequently be performed on several variables. For future costs and effectiveness data, a discount rate of $4 \%$ will be handled. In order to quantify the uncertainty surrounding the costs and effectiveness results, bootstrap analyses will be performed.

The cost-analysis from a societal perspective includes all health care costs and costs outside the health care sector. The cost-analysis will be performed by means of the microcosting method, whereby a detailed inventory of the resource use per patient will take place (Gold et al., 1996). Costs are calculated by multiplying resource utilization with the cost-price per unit. Health care costs refer to hospital costs and costs of other health care facilities. Hospital costs consist of personnel, material, capacity costs and overhead associated with the four programs and costs of (scheduled and unscheduled) outpatient hospital visits, telephone interviews and medical services (e.g. laboratory tests, bone scans, et cetera). Most of these costs are estimated by using existing resource registration systems and available cost prices through the financial departments of the participating centers. In case true cost prices are not readily available in all participating centers the existing cost-prices of the University Hospital Maastricht or official directive prices are used (Oostenbrink et al., 2004). Costs of telephone follow-up consist of personnel costs of the BCN and telephone costs. Costs associated with the EGP primarily consist of the personnel costs. Other health care costs include costs of visits to the general practitioner, medication, psychological and/or psychiatric (emergency) care. 
Costs outside the health care sector refer to out-of-pocket expenditures and productivity costs due to reductions in paid work and/or domestic activities. These costs are determined by a prospective cost diary, which is filled in by the patient at baseline, and thereafter at four time points $(3,6,12$ and 18 months after treatment) for a period of four weeks. Productivity losses due to absence from work are estimated by means of the friction cost method (Koopmanschap et al., 1995; Oostenbrink et al., 2004). In case of domestic or other unpaid activities, shadow prices will be used.

\section{DISCUSSION}

Current frequent follow-up is not meeting its intended aims, but does raise the burden on medical specialists, leading to high medical costs. Although studies investigating reduced follow-up have shown that there is no difference in time to detection of recurrence and patients perceived QoL, reduced follow-up has not yet been widely accepted and applied. Medical specialist may feel that patients need constant reassurance and patients have the false expectation that frequent follow-up will improve survival (de Bock et al., 2004b). Improvement of psychosocial support and information to patients could lead to a better acceptance of reduced follow-up. The trial reported in this paper combines these two aspects. By performing an economic evaluation parallel to the trial, the ultimate aim is to find an alternative and more cost-effective follow-up strategy. We expect the educational group program to help breast cancer patients and their partners in dealing effectively and efficiently with the consequences of a recent diagnosis and treatment of a potentially fatal disease. This increase in perceived behavioral control is expected to improve QoL. The nurse-led telephone calls are expected to render visits to the outpatient clinic superfluous, which may subsequently reduce the anxiety experienced by patients for each follow-up visit. Looking at costs, the telephone follow-up is expected to be cost-saving, due to fewer visits to the medical specialist. The EGP is expected to result in a slight cost-increase per patient in the first follow-up year, as compared to standard follow-up. However, due to the expected increase in QoL it may yield 1) reduced health care costs and 2) possibly lower costs due to a reduction in productivity losses.

The close cooperation with many centers enables us to reach a representative study population of women treated for primary breast cancer and enhances the generalizability of the trial results. In addition, BCNs are systematically trained to perform telephone follow-up and the EGP is based on standardized presentations. If one or both interventions appear to be successful, they are easily transferable to other settings. Finally, results may be extended to other cancer patients, but a further study would be required. 


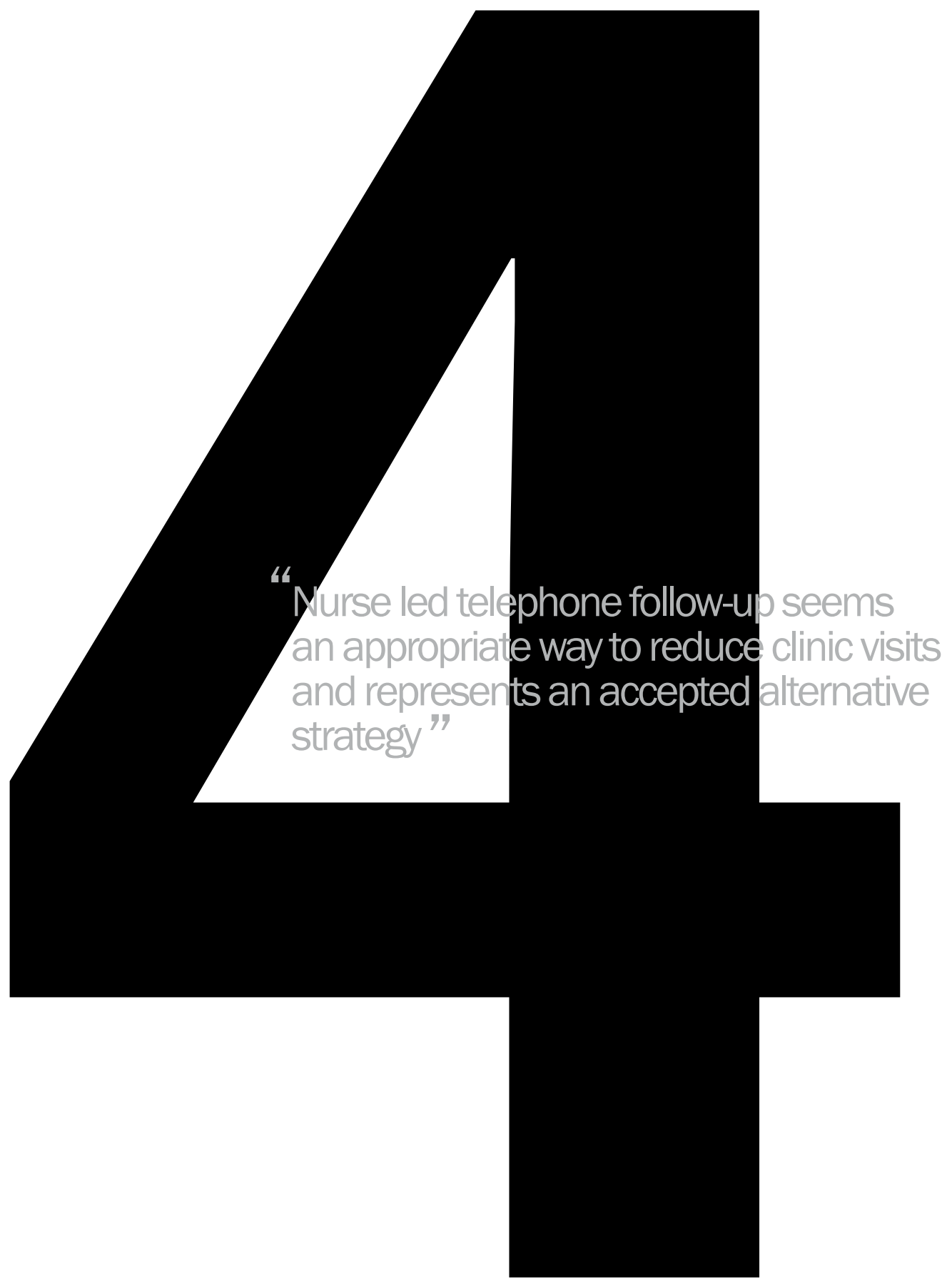




\section{Nurse-led telephone follow-up and an educational group program after breast cancer treatment: results of a $2 \times 2$ randomized clinical trial}

KIMMAN ML, DIRKSEN CD, VOOGD AC, FALGER P, GIJSEN BCM, THURING M, LENSSEN A, VAN DER ENT F, VERKEYN J, HAEKENS C, HUPPERETS P, NUYTINCK JKS, VAN RIET Y, BRENNINKMEIJER SJ, SCHEIJMANS L, KESSELS A, LAMBIN Ph, BOERSMA LJ.

UNDER REVIEW

\section{ABSTRACT}

[Objective] To investigate whether frequent hospital follow-up in the first year after breast cancer treatment might partly be replaced by nurse-led telephone follow-up without deteriorating health-related quality of life (HRQoL), and whether a short educational group program (EGP) would enhance HRQoL. [Design] Pragmatic, 2x2 factorial, randomized controlled trial. [Setting] Multicenter study in the Netherlands. [Participants] Between July 2005 and March 2008, seven hospitals and two radiation therapy clinics included 320 breast cancer patients who were treated with curative intent. [Interventions] Participants were randomized to follow-up care as usual (3-monthly outpatient clinic visits), nurseled telephone follow-up, or the former strategies combined with an educational group program. [Main outcome measures] The primary outcome for both interventions was HRQoL, measured by the EORTC QLQ-C30 questionnaire. Secondary outcomes were role and emotional functioning, and feelings of control and anxiety. [Results] Data of 299 patients were available for evaluation. In the primary analysis, 150 patients allocated to nurse-led telephone were compared to 149 patients in hospital follow-up, and 149 patients allocated to EGP were compared to 150 patients not allocated to the EGP. There was no significant difference in HRQoL between nurse-led telephone and hospital follow-up at 12 months after treatment ( $p=0.42 ; 95 \% \mathrm{Cl}$ for difference: -1.93 to 4.64 ) and neither between follow-up with or without EGP ( $p=0.86$; $95 \% \mathrm{Cl}$ for difference: -3.59 to 3.00). Furthermore, no differences were found in role and emotional functioning, and feelings of control and anxiety (all p-values $>0.05$ ). [Conclusions] Replacing most hospital follow-up visits in the first year after breast cancer treatment by nurse-led telephone follow-up does not impede patient outcomes. Hence, nurseled telephone follow-up seems an appropriate way to reduce clinic visits. 


\section{INTRODUCTION}

After curative treatment for breast cancer women commonly attend regular follow-up examinations. In most countries, follow-up is rather frequent (2-4 times annually) in the first 2-3 years; thereafter frequency is reduced to once a year (Khatcheressian et al., 2006; Pestalozzi \& Castiglione, 2008). The aim of these follow-up examinations is to detect local disease recurrence or a second primary breast cancer, but also to provide information and psychosocial support (Khatcheressian et al., 2006; Pestalozzi et al., 2005). However, there has been much debate regarding the value of this routine follow-up of women with breast cancer. Current follow-up patterns depend heavily on expensive and scarce specialized knowledge for routine history taking and for physical examinations, but do not always provide optimal care for patients (Grunfeld, 2009; Kimman et al., 2007a; Montgomery et al., 2009; Montgomery et al., 2007; Pennery \& Mallet, 2000).

Alternative follow-up strategies have been proposed, such as follow-up by general practitioners (Grunfeld et al., 2006), nurse-led follow-up (Koinberg et al., 2004) or telephone follow-up (Beaver et al., 2009b; Montgomery, 2009). Although studies have shown that these alternative follow-up models can be equally effective as traditional hospital followup, as measured by a variety of outcomes, these models are not yet widely applied in clinical practice. Results may not have been sufficiently convincing, or perhaps medical specialists believe that patients need frequent reassurance. Moreover, patients may have false expectations that frequent follow-up will result in better overall survival (de Bock et al., 2004b). Improvement of psychosocial support and education of patients may lead to better acceptance by both patients and specialists of a reduced follow-up policy (Koinberg et al., 2006). A number of studies have been performed using various group intervention formats in order to provide psychosocial support for breast cancer patients. However, most interventions focused on intensive psychological support (10 sessions or more) and showed varying results and often high refusal rates (Clark et al., 2003; Helgeson et al., 1999).

Since the educational aspects of these group interventions were most successful (Helgeson et al., 2000), a short educational group program (EGP) was developed for the study presented in this paper, specifically focusing on psychosocial and physical sequelae of breast cancer treatment. We hypothesized that the EGP would increase health-related quality of life (HRQoL) in the first year after treatment. In addition, nurses were trained to perform telephone follow-up to replace most of the hospital visits in the first year after treatment. We hypothesized that HRQoL of patients receiving nurse-led telephone followup would be at least similar to HRQoL of patients after hospital follow-up. To test these hypotheses we performed a randomized controlled trial with a $2 \times 2$ factorial design was performed. The aim of the current paper is to report on the effects of nurse-led telephone follow-up and the EGP on HRQoL and several other psychological aspects. Data on costeffectiveness obtained in this trial will be reported in a separate paper. 


\section{METHODS}

\section{Recruitment, design and sample}

Details of the randomized controlled trial (RCT) design and protocol execution have been reported previously (ISRCTN 74071417) (Kimman et al., 2007b). In sum, the study was a multicentre RCT with a 2x2 factorial design. Between 2005 and 2008, 320 females were recruited through seven hospitals and two radiotherapy clinics in the South of the Netherlands. Participants were eligible for inclusion if they had completed breast cancer treatment with curative intent less than six weeks prior to randomization with a WHO performance score between 0-2, and were fluent in speaking and reading Dutch. Exclusion criteria were distant metastases and/or participation in another clinical trial or medical illness requiring more frequent follow-up. All eligible patients received detailed study information from their medical specialist. This study information included information on the purpose and effectiveness of breast cancer follow-up, and signs and symptoms of possible recurrences.

After approximately one week a research assistant contacted the patient by telephone to answer any questions and, when a patient was willing to participate, ask to provide written informed consent. Participants were then randomly assigned to one of four followup strategies (study arms) during the first 18 months after treatment; i.e. 1) hospital follow-up as usual: five outpatient clinic visits in the first 18 months (at 3, 6, 9, 12 and 18 months), including a mammography at 12 months; 2) nurse-led telephone follow-up: a mammography at 12 months combined with an outpatient clinic visit, and telephone interviews by a breast care nurse (BCN) at the same time points as for usual follow-up (i.e. 3, 6, 9 and 18 months); 3) arm 1 plus educational group program (EGP); 4) arm 2 plus EGP. Study arms were combined for analysis as follows: hospital follow-up (arms 1 and 3) compared to nurse-led telephone follow-up (arms 2 and 4); and EGP (arms 3 and 4) compared to no EGP (arms 1 and 2).

Randomization by minimization (Taves, 1974) was performed by the independent Comprehensive Cancer Centre Limburg using a computerized randomization programme (ALEA). Patients were pre-stratified by hospital and treatment modality (surgery, surgery + radiotherapy, surgery + chemotherapy, and surgery + radiotherapy + chemotherapy). The study protocol was approved by the Medical Ethical Review Board of MAASTRO Clinic (NL). All participating centres signed local feasibility declarations, according to Dutch law and regulations, prior to inclusion of the first patient.

\section{Procedures and interventions}

For patients randomized to hospital follow-up, care was provided by the surgeon, medical oncologist, radiation oncologist and/or breast care nurse (BCN). Follow-up visits consisted of medical history taking and physical examination; scheduled duration was $10 \mathrm{~min}$ utes. For patients randomized to telephone follow-up, follow-up was performed by a BCN specifically trained for this study. The telephone interview consisted of a semi-structured questionnaire including screening for physical -especially loco-regional- and psychological 
symptoms, treatment side effects, compliance with hormonal therapy plus open discussion of these issues. Furthermore, the BCN informed about patients' general well-being, family life and work reintegration. Time scheduled for the telephone interview was 20 minutes.

In study arms 3 and 4, patients and their partners were invited to participate in the EGP which consisted of two interactive group sessions of 2.5 hours each (with two weeks in between). The EGP took place regularly so that each patient could participate within three months after completion of treatment. A BCN provided information on possible treatment side effects and topics such as signs and symptoms of possible recurrences, prostheses and fatigue. A health care psychologist addressed psychological and social consequences of breast cancer, particularly anxiety and changes in family and social role patterns, and discussed psychological coping strategies. The EGP was concluded with a relaxation exercise; also, participants received a booklet encompassing all topics discussed in the EGP.

\section{Outcome measures}

\section{Primary outcome measure}

The primary outcome for both interventions was HRQoL at 12 months after randomization, measured by EORTC QLQ-C30. This self-administered disease-specific HRQoL questionnaire is validated for oncological clinical research (Bottomley \& Aaronson, 2007; Fayers \& Bottomley, 2002) and has a validated Dutch version available (Aaronson et al., 1993). The EORTC QLQ-C30 consists of 30 items and provides scores on multi-item functional subscales (e.g. role, emotional functioning), multi-item symptom scales (e.g. fatigue, pain), a HRQoL (global health) subscale and a number of single items (e.g. sleep disturbance). The HRQoL subscale consists of two items: (1) How would you rate your overall health during the past week? and; (2) How would you rate your overall quality of life during the past week? Items are rated on a 7-point Likert-scale, ranging from "very bad" to "excellent". Scores on HRQoL and functional subscales range from 0 to 100, with higher scores representing higher levels of functioning and HRQoL.

\section{Secondary outcome measures}

Secondary outcomes were emotional and role functioning, anxiety and perceived feelings of control. Emotional and role functioning were measured by EORTC QLQ-C30. The emotional functioning subscale consists of four items concerning presence of feelings of depression, tension, loss of concentration and fear. The role functioning subscale concerns the (dis)ability to perform daily activities. Both subscales are rated on a 4-point Likert scale and transformed to a score between 0 and 100.

Anxiety was measured by the state anxiety scale of the validated Dutch version of the State-Trait Anxiety Inventory (STAI) (van der Ploeg, 1980). This 20-items questionnaire measures the contemporary condition of "state anxiety" initiated by current life events. Scores range from 20-80, a higher score indicating greater anxiety. 
Perceived feelings of control were measured by the Dutch version of the Mastery Scale (Pearlin \& Schooler, 1978). This questionnaire consists of seven statements about feelings of control (e.g. "I have little control over the things that happen to me"). A separate statement about experienced emotional support from partner was added. Rating alternatives ranged from "strongly disagree" to "strongly agree" and average scores were calculated (range from 0 to 5), with higher scores representing higher perceived feelings of control.

\section{Statistical analysis}

Sample size calculations were based on HRQoL measured by EORTC QLQ-C30 (Aaronson et al., 1993). A baseline score of 64 with a standard deviation of 17 was considered as a starting point (Gijsen \& Koppejan-Rensenbrink, 2003; Nagel et al., 2001). An analysis of the clinical significance of HRQoL changes showed that patients indicate a change between 5-10 to be small, between 10-20 to be moderate, and of more than 20 to be large (Osoba et al., 1998). Consequently, we considered a change smaller than 5 to mean no change. A power analysis on our study design required 320 breast cancer patients in order to demonstrate that HRQoL of patients with nurse-led telephone follow-up would be at least similar (i.e. $<5$ points difference) to patients in the hospital follow-up (non-inferiority analysis; power 80\%, $\alpha$ 0.05). Also, with 320 patients, a moderate improvement in HRQoL (i.e. > 10 points difference) in patients attending the EGP could be detected (superiority analysis; power $95 \%, \alpha<0.01$ ). A dropout rate of $10 \%$ was accounted for in these calculations. Sample size was calculated for main effects only and assumed no interaction between the two interventions.

Patients completed questionnaires before randomization at baseline, and at 3, 6, 12 and 18 months after respective treatments. Data were entered and analyzed with SPSS 17.0. Missing data were imputed by means of Rubin's multiple imputation procedure using the defaults in SPSS 17.0, generating five datasets (Schafer, 1999; Sterne et al., 2009). All analyses were performed with each of the five data sets, and results were pooled Differences in patient characteristics between participants and non-participants were compared using the $\mathrm{X}^{2}$ test and independent sample t-test. The primary analysis assessed the above pre-defined effects of both interventions. Patients remained in the study arm to which they were assigned, whether or not they completed the intervention given to the arm. Additionally, we included an interaction effect to test for interaction/mutual effect modification between interventions. Regression analyses were used to predict primary and secondary outcome differences at 12 months after randomization by including or excluding interventions. Linear mixed models were fitted with EGP (yes/no) and telephone follow-up (yes/no) as fixed factors and patient as random factor. In addition, recruitment hospital, age, education, time since end of treatment and treatment modality were included as covariates. Primary and secondary outcomes were adjusted for baseline differences. 
Since protocol violation may bias the trial (in either direction), per protocol analyses including only patients who properly followed the study protocol were also performed and reported, as recommended by Piaggio et al. (2006) and Jones et al. (1996). Furthermore, explorative subgroup analyses were performed according to level of anxiety, age, level of education, use of chemotherapy and level of support from partner. Analyses were performed by creating an interaction term between the intervention and subgroup and testing the significance of the interaction in the regression model.

\section{RESULTS}

\section{Patients}

Between June 2005 and March 2008, 320 of 881 eligible patients (36.4\%) agreed to participate in the study (figure 1). Nearly half of the patients who refused to participate $(n=240)$ agreed to fill out the baseline questionnaire for comparison with participants. At baseline, participants did not differ from non-participants with respect to education, marital status, HRQoL, emotional functioning and feelings of anxiety (all p-values $>0.05$ ). However, participants were significantly younger than non-participants (mean age 60 years $(S D=10.2), p<0.001)$.

Throughout the trial, 21 patients (6.5\%) were lost to follow up for various reasons (e.g. development of metastases, recurrence, or three or more missing questionnaires) (figure 1).

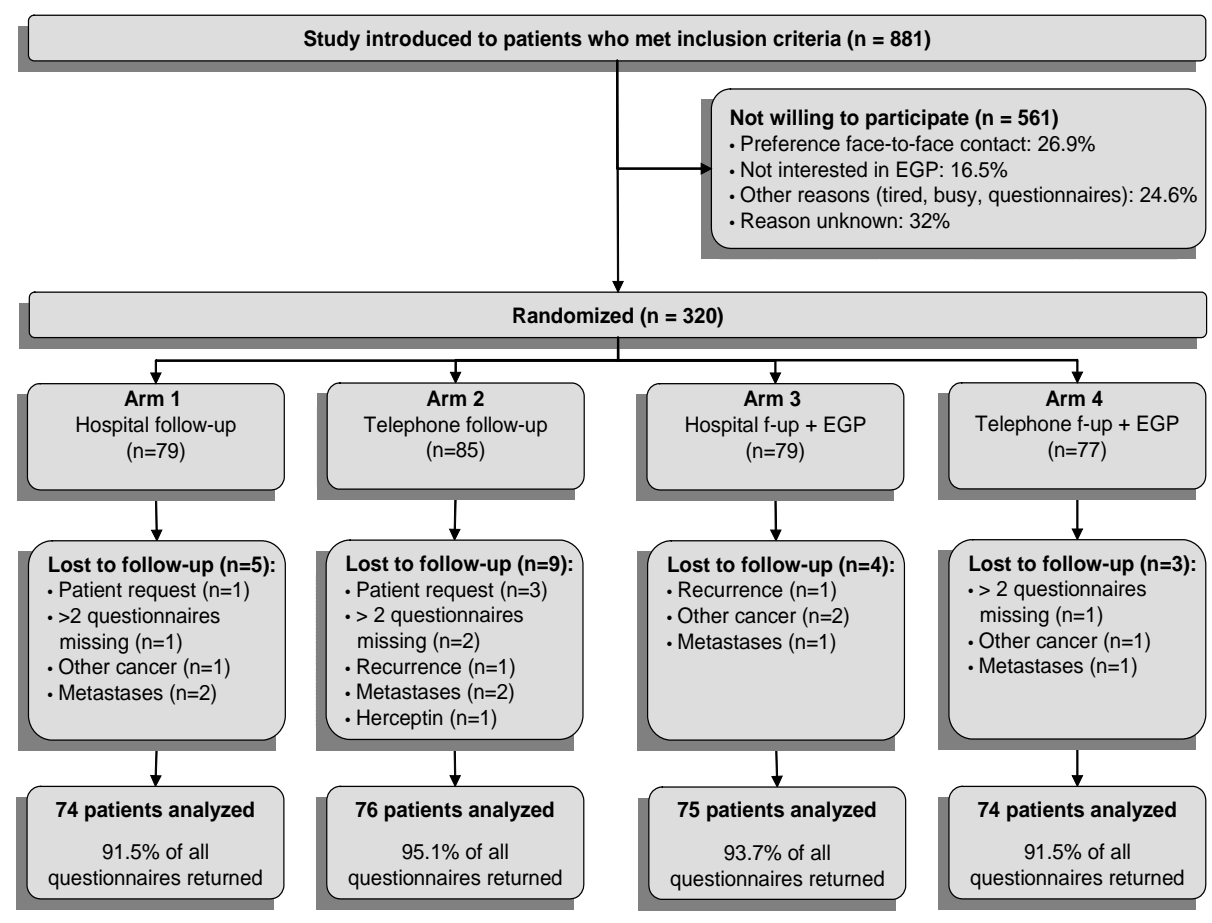

Figure 1. Flow of participants through trial 
Data from 299 patients were available for the analysis; 149 patients randomized to hospital follow-up and 150 patients randomized to telephone follow-up; and 149 patients randomized to follow-up with EGP and 150 patients randomized to follow-up without EGP, at three months. Baseline demographic and treatment characteristics were similar for patients randomized to telephone and hospital follow-up and for those with or without EGP (table 1). Mean sample age was 56 years $(S D=9.9)$.

Table 1. Baseline and sociodemographic characteristics of participants in intervention (telephone followup or educational group program (EGP) and control arms. Values are numbers (percentages) unless stated otherwise. $\mathrm{RT}=$ radiotherapy and $\mathrm{CH}=$ chemotherapy.

\begin{tabular}{|c|c|c|c|c|c|}
\hline & $\begin{array}{l}\text { Hospital } \\
\text { f-up } \\
(n=149)\end{array}$ & $\begin{array}{l}\text { Telephone } \\
\text { f-up }(n=150)\end{array}$ & $\begin{array}{l}\text { EGP } \\
(n=149)\end{array}$ & $\begin{array}{l}\text { No EGP } \\
(n=150)\end{array}$ & $\begin{array}{l}\text { All partici- } \\
\text { pants } \\
(n=299)\end{array}$ \\
\hline \multicolumn{6}{|l|}{ Age at recruitment } \\
\hline Mean in years (SD) & $56.2(10.7)$ & $55.5(9.0)$ & $55.3(10.4)$ & $56.3(9.4)$ & $55.8(9.9)$ \\
\hline Range in years & $23-78$ & $34-75$ & $23-76$ & $34-78$ & $23-78$ \\
\hline \multicolumn{6}{|l|}{ Level of education } \\
\hline Low & $45(30.2)$ & $57(38.0)$ & $51(34.2)$ & $51(34.0)$ & $102(34.1)$ \\
\hline Middle & $62(41.6)$ & $56(37.3)$ & $60(40.3)$ & $58(38.7)$ & 118 (39.5) \\
\hline High & $42(28.2)$ & $37(24.7)$ & $38(25.5)$ & $41(27.3)$ & $79(26.4)$ \\
\hline \multicolumn{6}{|l|}{ Marital status } \\
\hline Married & $109(73.2)$ & $103(68.7)$ & $104(69.8)$ & $108(72.0)$ & $212(70.9)$ \\
\hline Unmarried & $16(10.7)$ & $17(11.3)$ & $17(11.4)$ & $16(10.7)$ & $33(11.0)$ \\
\hline Cohabiting & $9(6.0)$ & $20(13.3)$ & $13(8.7)$ & $16(10.7)$ & $29(9.7)$ \\
\hline Widowed & 15 (10.1) & $10(6.7)$ & 15 (10.1) & $10(6.7)$ & $25(8.4)$ \\
\hline \multicolumn{6}{|l|}{ Tumor stage } \\
\hline Stage I & 91 (61.1) & $90(60.0)$ & $85(57.0)$ & $96(64.0)$ & $181(60.5)$ \\
\hline Stage Ila & $35(23.5)$ & $34(22.7)$ & $36(24.2)$ & $33(0.22)$ & 69 (23.1) \\
\hline Stage IIb & $8(5.4)$ & $13(8.7)$ & $13(8.7)$ & $8(5.3)$ & $21(7.0)$ \\
\hline Stage III & $13(8.7)$ & $11(7.3)$ & $12(8.1)$ & $13(8.7)$ & $24(8.0)$ \\
\hline Unknown & $2(1.3)$ & $2(1.3)$ & $3(2.0)$ & - & $4(1.3)$ \\
\hline \multicolumn{6}{|l|}{ Treatment modality } \\
\hline Surgery & $15(10.1)$ & $14(9.3)$ & $13(8.7)$ & $16(10.7)$ & $29(9.7)$ \\
\hline Surgery + RT & $89(59.7)$ & 89 (59.3) & 91 (61.1) & $87(58.0)$ & $178(59.5)$ \\
\hline Surgery $+\mathrm{CH}$ & $7(4.7)$ & $8(5.3)$ & $8(5.4)$ & $7(4.7)$ & $15(5.0)$ \\
\hline Surgery + $\mathrm{RT}+\mathrm{CH}$ & $38(25.5)$ & $39(26.0)$ & $37(24.8)$ & $40(26.7)$ & $77(25.8)$ \\
\hline Hormonal therapy & $50(33.6)$ & $44(29.3)$ & $48(32.2)$ & $46(30.7)$ & $94(31.4)$ \\
\hline \multicolumn{6}{|l|}{ Health scores (mean (SD)) } \\
\hline Health-related quality of life & $70.5(17.8)$ & $67.2(19.0)$ & 70.5 (18.9) & $67.3(17.9)$ & $68.9(20.1)$ \\
\hline Emotional functioning & $77.7(20.4)$ & $69.9(25.7)$ & $75.8(23.6)$ & $71.8(23.3)$ & $73.8(23.5)$ \\
\hline Role functioning & $68.6(29.3)$ & $62.6(27.8)$ & $67.2(29.9)$ & $64.0(27.4)$ & $65.6(28.7)$ \\
\hline Feelings of anxiety & $39.3(11.3)$ & $40.9(11.8)$ & $39.0(11.5)$ & $41.2(11.6)$ & 40.1 (11.6) \\
\hline Feelings of control & $2.6(0.8)$ & $2.6(0.8)$ & $2.5(0.7)$ & $2.6(0.8)$ & $2.6(0.8)$ \\
\hline
\end{tabular}

\section{Compliance to the protocol}

Ten patients randomized to telephone follow-up preferred to receive hospital follow-up instead, and 20 patients with telephone follow-up received only one telephone follow-up contact, which was considered as protocol violation. Hence, 120 out of 150 patients in 
the telephone follow-up arms, received telephone follow-up according to protocol. The 30 patients who violated the protocol did not differ from other patients in the telephone followup group regarding age, education, treatment modality, role functioning and feelings of control at baseline (all p-values > 0.05). However, their HRQoL and emotional functioning were significantly lower and feelings of anxiety higher than protocol compliers ( $p$-values of respectively $0.02,0.01$ and 0.01 ).

Ten out of 149 patients randomized to EGP did not attend the meetings. These patients did not differ from those attending the EGP regarding age, education, treatment modality and all outcome measures (all p-values > 0.05). Seventy-eight patients (52\%) were accompanied by their partner at one or both meetings.

\section{Primary outcome: health-related quality of life (HRQoL)}

Intention to treat and per protocol analyses showed almost identical results (table 2). Results from the intention to treat analyses are discussed below. There was no significant difference in HRQoL between nurse-led telephone and hospital follow-up at 12 months after treatment $(p=0.42)$. The $95 \%$ confidence interval for difference between mean HRQoL scores at 12 months after treatment was -1.93 to 4.64 . A positive mean difference indicates higher levels of functioning for nurse-led telephone follow-up, while a negative difference indicates higher levels of functioning in the hospital follow-up. Yet, HRQoL significantly improved over time $(p=0.01)$, but without significant differences in slope of improvement between both follow-up groups $(p=0.41)$ (figure 2 ).

Similarly, there was no significant difference in HRQoL between follow-up with or without EGP at 12 months after treatment $(p=0.86)$. The $95 \%$ confidence interval for difference between mean HRQoL scores at 12 months was -3.59 to 3.00. Again, there was no significant difference in slope of improvement between follow-up groups $(p=0.10)$ (figure 2). Finally, there was no significant interaction effect between EGP and nurse-led telephone follow-up with respect to HRQoL $(p=0.50)$.
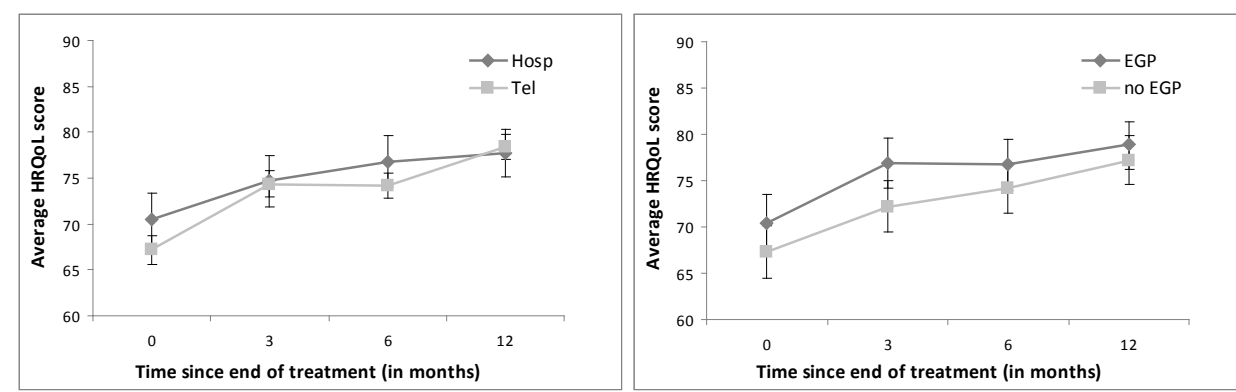

Figure 2. Average health-related quality of life (HRQoL) scores on the EORTC QLQ-C30 questionnaire at baseline, and 3, 6 and 12 months after treatment: telephone versus hospital follow-up (left) and an educational group program (EGP) versus no EGP (right). Error bars represent the $95 \%$ confidence interval. 
Table 2. Outcome findings by study group adjusted for treatment, hospital, outcome variable at baseline, age, educational level and time since treatment. Intention to treat analysis: $n=150$ for telephone $f$-up, $n=149$ for hospital f-up; $n=149$ for EGP, $n=150$ for no EGP. Per protocol analysis: $n=120$ for telephone f-up, $n=159$ for hospital $f$-up; $n=139$ for EGP, $n=160$ for no EGP.

\section{Estimated} difference
95\% Confidence Interval for difference ${ }^{a}$

\section{Intention to treat analysis}

\section{Health-related quality of life}

Telephone f-up vs hospital f-up

EGP vs no EGP

1.35

$-0.29$

\section{Emotional functioning}

Telephone f-up vs hospital f-up

EGP vs no EGP

2.23

$-1.66$

Role functioning

Telephone f-up vs hospital f-up

EGP vs no EGP

2.56

1.23

Feelings of anxiety

Telephone f-up vs hospital f-up

EGP vs no EGP

$-0.84$

$-0.10$

Perceived feelings of control

Telephone f-up vs hospital f-up

EGP vs no EGP

$-0.07$

0.00

0.27

$-0.75$

1.56

$-1.47$

2.28

1.37

$-0.13$

$-0.40$

$-0.09$

$-0.02$
-1.93 to 4.64

-3.59 to 3.00

-1.50 to 5.96

-5.32 to 2.00

-2.12 to 7.24

0.61

-3.45 to 5.92

-2.78 to $1.11^{b}$

-2.07 to $2.15^{b}$

-0.22 to 0.07

-0.15 to 0.15
0.42

0.86

0.24

0.37

0.28

0.40

0.92

0.32

p-value

0.99

\footnotetext{
a Positive differences imply a higher level of functioning in the telephone and EGP group.

${ }^{\mathrm{b}}$ Negative differences imply less anxiety in the telephone and EGP group.
}

\section{Secondary outcomes}

Role and emotional functioning, and feelings of control and anxiety improved over time, but there were no significant differences between patients randomized to telephone and hospital follow-up (table 2). Similarly, differences between having participated in EGP or not with respect to role and emotional functioning and feelings of control and anxiety, were not significant at 12 months after treatment. 


\section{Subgroup analyses}

Explorative subgroup analyses according to level of anxiety, age, education, use of chemotherapy, and level of support from partner did not identify specific subgroups of patients for whom the interventions would have been more effective than for others (data not shown).

\section{DISCUSSION}

Follow-up of breast cancer patients continues to be controversial despite almost two decades of research. Current frequent follow-up is not meeting its intended aims, but does raise the burden on specialized staff and hospital resources. This multicentre randomized controlled trial (RCT) showed that some hospital clinic visits in the first year after treatment could well be replaced by nurse-led telephone follow-up without a loss of HRQoL, a decrease in role and emotional functioning and feelings of control, nor an increase in feelings of anxiety. It was also found that an educational group program (EGP) did not lead to a significant improvement in HRQoL, or any other outcome measures.

In a recently published paper on this RCT we reported high patient satisfaction scores for both nurse-led telephone as well as hospital follow-up. Moreover, the numbers of hospital visits were substantially less in the telephone group compared to hospital followup (Kimman et al., 2010a). The present positive findings regarding nurse-led telephone follow-up are similar to those reported in earlier studies (Beaver et al., 2009b; Gulliford et al., 1997; Montgomery et al., 2008a). Hence, results from this RCT lend further support to acceptability, feasibility, and patient satisfaction associated with telephone follow-up instead of hospital follow-up. The RCT by Beaver and colleagues, comparing nurse-led telephone follow-up with hospital follow-up, showed that telephone follow-up significantly improved satisfaction and did not produce excess anxiety compared with hospital followup. Women were on average 20 months after end of treatment when entering the trial (Beaver et al., 2009b). Since in the present study women entered immediately after treatment both trials may be seen as complementary and both provide evidence that telephone follow-up appears appropriate for patients in different phases after treatment (Beaver et al., 2009b).

With respect to HRQoL and feelings of control and anxiety, the EGP did not meet our initial expectations. Based on these effectiveness results we would be hesitant to recommend unconditional implementation of the present EGP. However, an interesting finding in Beaver's study was that, although information needs of patients reduced over time, 20$30 \%$ still reported need for more information by the end of the study. This suggests that other methods to improve patients' information may be added to nurse-led telephone and hospital follow-up. Although not specifically investigated, the short-term EGP developed for our study could be an effective way to meet information needs of patients. Furthermore, a non-randomized study ( $n=96)$ by Koinberg and colleagues (2006), in which traditional hospital follow-up was compared to a short multidisciplinary educational program 
(replacing hospital visits) similar to our EGP, reported that both follow-up programs were equivalent in terms of well-being, aspects of self-care and coping abilities (Koinberg et al., 2006). Hence, our EGP might be a substitute for (some) traditional hospital follow-up visits. In our study, the EGP was complementary to hospital and telephone follow-up, and not yet used as a full substitute.

Our study achieved a relatively low participation rate of $36 \%$. However, considering that patients find hospital visits reassuring (Beaver \& Luker, 2005) and were asked to forego these visits and physical examinations for this study, as well as the fact that patients were made aware of the status quo and generally prefer this (Salkeld et al., 2000), this low participation rate was anticipated. Importantly, the cooperation with many centres enabled us to reach a representative study population of women treated for primary breast cancer, thus enhancing generalization of the results to a national level. Nevertheless, the low participation rate and considerable protocol violation in the telephone follow-up may suggest that instead of a one-size-fits-all approach, more individualized follow-up may have to be applied, taking into account specific patient preferences. Patients who violated the protocol had lower HRQoL and higher levels of anxiety at the end of treatment; therefore for these patients hospital follow-up may be more appropriate. Moreover, our recent study on preferences for follow-up in the first year after breast cancer treatment showed that preferences for the health care provider for follow-up and for participation in an EGP were very heterogeneous (Kimman et al., 2010b). Nevertheless, it must be realized that implementation of reduced or individualized follow-up strategies asks for a paradigm shift, requiring attitude change among both medical specialists and patients and efforts to prevent false expectations of follow-up efficacy. In addition, it is important to first explore whether a more individualized follow-up indeed shows improved outcomes in terms of satisfaction and HRQoL and is feasible in clinical practice.

In conclusion, replacement of most hospital follow-up visits in the first year after treatment by nurse-led telephone follow-up does not impede patient outcomes. Hence, nurse-led telephone follow-up seems an appropriate way to reduce clinic visits and represents an accepted alternative strategy. An EGP may be appropriate to address information needs, but it may not unequivocally affect positive HRQoL outcomes. 


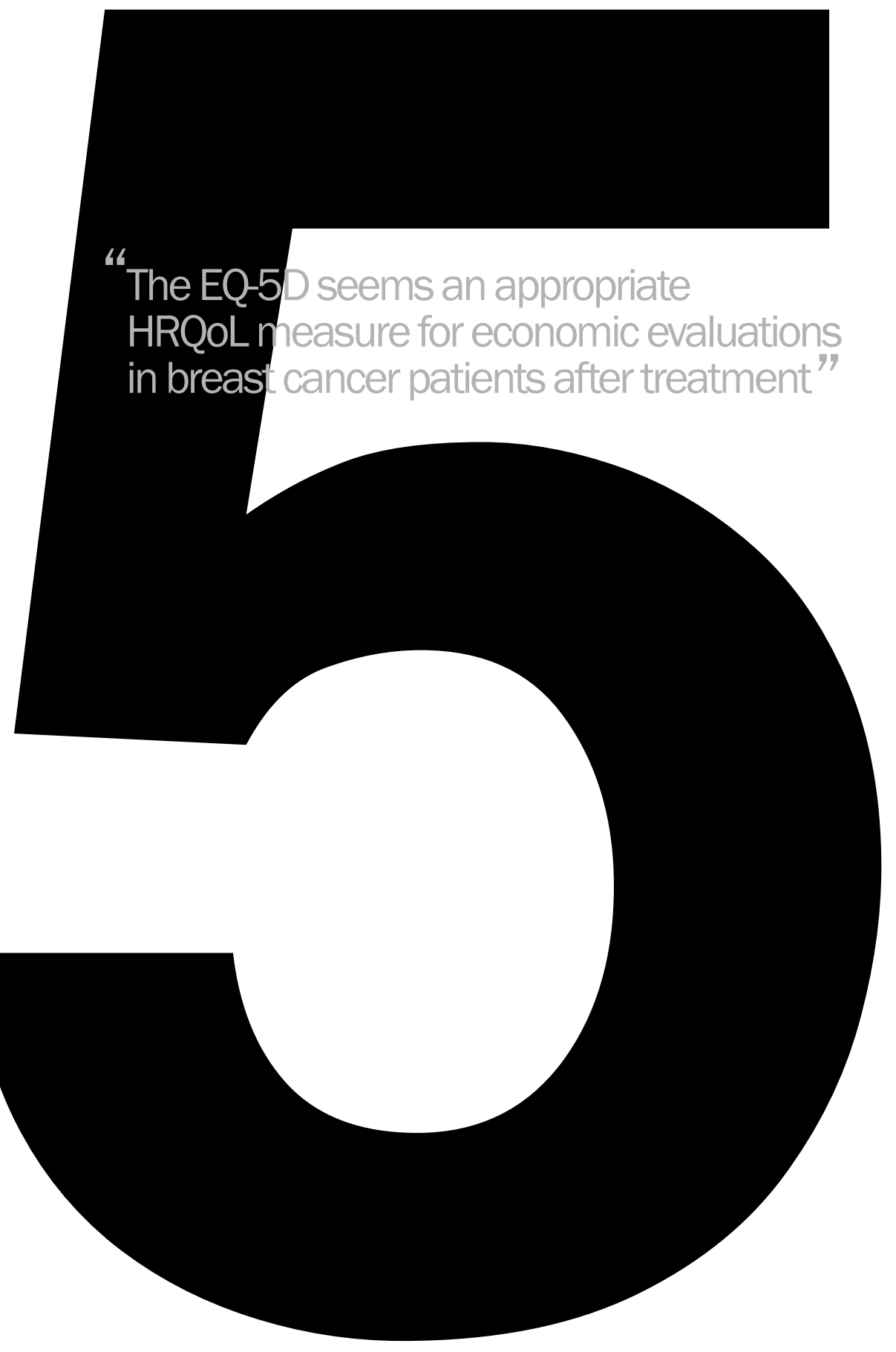




\title{
Responsiveness of the EQ-5D in breast cancer patients in their first year after treatment
}

KIMMAN ML, DIRKSEN CD, LAMBIN Ph, BOERSMA L.

HEALTH AND QUALITY OF LIFE OUTCOMES 2009;7(1):11

\begin{abstract}
[Background] The EQ-5D is a generic health-related quality of life (HRQOL) measure that is used for the purpose of economic evaluations of health interventions. Therefore, it has to be responsive to meaningful changes in health in the patient population under investigation. The aim of this study was to investigate the responsiveness of the EQ-5D in breast cancer patients in their first year after treatment. [Methods] The subscale global health of the disease-specific HRQoL measure EORTC QLQ-C30 was used as a reference instrument to determine meaningful changes in health and identify subgroups of patients: patients reporting a moderatelarge deterioration, small deterioration, a small improvement, moderatelarge improvement, or no change in health status. Responsiveness was evaluated by calculating standardized response means (SRMs) in the five subgroups of patients and performing analysis of variance procedures. The two HRQoL measures were filled out two weeks and one year after finalizing curative treatment for breast cancer ( $n=192)$. [Results] The EQ5D was able to capture both improvements and deteriorations in HRQoL. SRMs of the EQ VAS and EQ-5D Index were close to zero in the subgroup reporting no change and increased and decreased adequately in the subgroups reporting small and moderate changes. Additional analysis of variance procedures showed that the EQ-5D was able to differentiate between subgroups of patients with no change and moderate-large deterioration or improvement in health. [Conclusions] The EQ-5D seems an appropriate measure for the purpose of economic evaluations of health intervention in breast cancer patients after treatment.
\end{abstract}




\section{INTRODUCTION}

With an estimated 1.15 million new cases worldwide each year and a relatively good prognosis, breast cancer is the most prevalent cancer in the world today (Parkin et al., 2005). After curative treatment for breast cancer, women attend frequent follow-up visits to be examined for possible local or regional recurrence or a second primary breast tumor, and to receive psychosocial support (ESMO, 2001; Khatcheressian et al., 2006a). However, no strong evidence exists that regular follow-up is effective with regard to disease free survival or overall survival (de Bock et al., 2004a; Jacobs et al., 2001; te Boekhorst et al., 2001), or in providing psychosocial support (Allen, 2002; Pennery \& Mallet, 2000). Hence, the assessment of outcomes like patient satisfaction and health-related quality of life (HRQoL) is common practice in clinical oncology trials investigating alternative followup strategies and psychosocial interventions for breast cancer survivors (Antoni et al., 2001; Grunfeld et al., 1999a; Helgeson et al., 2001; Kimman et al., 2007a; Kimman et al., 2007b; Koinberg et al., 2004; Marcus et al., 1998; Meneses et al., 2007; Sandgren \& McCaul, 2003). Given the high prevalence of breast cancer and budget constraints in health care, it is also important to understand the impact of alternative strategies on economic outcomes. Therefore, clinical trials are increasingly incorporating generic HRQoL measures, such as the EQ-5D, for the purpose of economic evaluations (Pickard et al., 2007). The EQ-5D is a standardized multi-dimensional health state classification system. It generates a single index score for each health state (EuroQoL-Group, 1990). Index scores, in turn, can be used to calculate quality adjusted life years (QALYs), which is the most preferred summary outcome measure in economic evaluations (Drummond et al., 2005).

A substantial and growing body of literature regarding the usefulness of the EQ-5D in cancer has emerged, supporting its validity and reliability (Pickard et al., 2007). However, the responsiveness of the EQ-5D, defined as its ability to capture true underlying changes in the patients' health status over time (Terwee et al., 2003), is highly dependent on patient population and setting. In comparison with disease-specific instruments, the responsiveness of the EQ-5D was found to be comparable in one study (Krabbe et al., 2004), but more often it is found to be less responsive than disease-specific instruments (Eurich et al., 2006; Krahn et al., 2007; van de Willige et al., 2005; Wiebe et al., 2003). Hence, the usefulness of the EQ-5D may be limited if it is not able to detect changes in health status in the patient population under investigation.

To our knowledge, the responsiveness of the EQ-5D has not yet been examined in breast cancer patients after treatment. Therefore, we use data from a randomized clinical trial investigating several follow-up strategies for curatively treated breast cancer patients (Kimman et al., 2007b) to address whether the EQ-5D is responsive to changes in HRQoL in a population of breast cancer patients in their first year after treatment. 


\section{METHODOLOGY}

\section{Study population}

Participants were enrolled in a randomized clinical trial investigating the cost-effectiveness of nurse-led telephone follow-up and a short educational group program after curative treatment for breast cancer (MaCare trial, ISRCTN 74071417) (Kimman et al., 2007b). Patients in the trial were all female, treated for breast cancer with curative intent, and had no concomitant tumors or comorbidity requiring hospital visits. There were no age restrictions. Patients were included in the trial after finalizing treatment and after giving written informed consent. Treatment included surgery and/or radiotherapy and/ or chemotherapy. Follow-up appointments took place at 3,6,9 and 12 months after treatment. For the purpose of studying the responsiveness of the EQ-5D, patients who had had their 12 months follow-up were eligible. The EQ-5D and the disease-specific EORTC QLQ-C30 were sent to patients at home two weeks after the end of treatment (TO) and twelve months after treatment (T1). Of 220 eligible patients, 29 patients failed to complete both instruments at both measurements due to either random missings within the instruments $(n=19)$ or because they were a study drop-out $(n=10)$. A total of 192 patients were therefore included in the analysis. Their demographic and clinical characteristics can be found in table 1 . Patients were analyzed regardless of follow-up strategy in the trial.

The MaCare trial was approved by the Independent Ethics Committee of MAASTRO Clinic.

Table 1. Characteristics of participants $(n=192)$

$\begin{array}{ll}\text { Characteristic } & \text { Descriptive statistic } \\ \text { Age } & \\ \text { Mean (SD) } & 55.8(10.1) \text { yrs } \\ \text { Range } & 23-79 \text { yrs } \\ \text { Level of education } & \\ \text { Low } & 69(35.9 \%) \\ \text { Middle } & 96(50.0 \%) \\ \text { High } & 27(14.1 \%) \\ \text { Tumor stage } & \\ \text { Stage I } & 99(51.6 \%) \\ \text { Stage II } & 61(31.8 \%) \\ \text { Stage III } & 17(8.6 \%) \\ \text { Unknown } & 15(7.8 \%) \\ \text { Treatment modality } & 17(8.9 \%) \\ \text { Surgery } & 107(55.7 \%) \\ \text { Surgery and radiotherapy } & 13(6.8 \%) \\ \text { Surgery and chemotherapy } & 55(28.6 \%) \\ \text { Surgery and radiotherapy and chemotherapy } & 65(34 \%) \\ \text { Hormonal therapy } & \end{array}$




\section{HRQoL Instruments \\ EQ-5D}

The EQ-5D is a short generic health-related quality of life instrument that consists of two parts: a self-classifier and a Visual Analogue Scale (EQ VAS). The self-classifier comprises five items relating to problems in the following domains: mobility, self-care, usual activities, pain/discomfort and anxiety/depression (EuroQoL-Group, 1990). Each domain has three levels, namely, "no problems", "some problems" and "severe problems". Combinations of these categories define a total of 243 health states. Dolan et al (Dolan, 1997) have presented 42 of these health states to approximately 3000 members of a representative sample of the UK general population, which were valued using the time-trade-off (TTO) technique. Based on these valuations, for each health state a utility score can be deducted, called the EQ-5D Index score. These EQ-5D Index scores may vary between - 0.59 (worst health) and 1.00 (perfect health). On the EQ VAS respondents can indicate their overall self-perceived health state on a scale ranging from 0 to 100 , where 0 is equivalent to the worst imaginable health state and 100 is equivalent to the best imaginable health state.

\section{EORTC QLQ-C30}

The EORTC QLQ-C30 from the European Organization for Research and Treatment of Cancer is a self-administered disease-specific HRQoL questionnaire and is validated for oncology clinical research (Aaronson et al., 1993; Bottomley \& Aaronson, 2007; Fayers \& Bottomley, 2002). It has also been validated (McLachlan et al., 1998) and found to be responsive (Osoba et al., 1994) specifically in breast cancer patients and is widely used in breast cancer research investigating HRQoL after treatment (Arndt et al., 2005; Arndt et al., 2004; Goodwin et al., 2004; Helgesson et al., 2007; Schou et al., 2005; Waldmann et al., 2007). The HRQoL questionnaire consists of 30 items. After transformation, the EORTC QLQ-C30 has several multi-item functional subscales (e.g. physical, emotional functioning), multi-item symptom scales (e.g. fatigue, pain), a global health subscale, and single items to assess symptoms (e.g. sleep disturbance). Scores on the functional and global health scales range from 0 to 100 , where a higher scale score represents a higher level of functioning and therefore HRQoL.

\section{Analyses of responsiveness}

To assess the responsiveness of the EQ-5D three steps were taken, following recommendations recently published by Revicki et al. (2008). First, a criterion, or anchor, that is related to the measure under investigation, was selected to identify whether patients had changed (either improved or worsened) over time. Second, when the relationship between the anchor and EQ-5D was confirmed, patients were classified into subgroups according to changes in their health status. Third, to examine responsiveness, statistical indicators for change were calculated and analysis of variance procedures were performed. 


\section{Step 1: Selecting an anchor; global health of the EORTC QLQ-C30}

Selecting anchors should be based on criteria of relevance for the disease indication, clinical acceptance and validity, and evidence that the anchors have some relationship with the measure under investigation (Revicki et al., 2008). For this study, the subscale global health of the EORTC QLQ-C30 was proposed as a criterion for clinical change. The global health subscale consists of two items: (1) How would you rate your overall health during the past week? and; (2) How would you rate your overall quality of life during the past week?

Correlations between global health scores and the EQ-5D Index and EQ VAS were calculated to examine whether the anchor was acceptable. It is recommended that $0.30-0.35$ is used as a correlation threshold to define acceptable association between an anchor and a change score on the HRQoL outcome measure (Revicki et al., 2008).

\section{Step 2: Classifying patients into subgroups}

Change scores on global health of the EORTC QLQ-C30 were used to identify subgroups of patients. In an analysis of the clinical significance of changes in HRQoL, Osoba et al. (1998) showed that patients judge a change between 5-10 on the global health scale of the EORTC QLQ-C30 to be small, between 10-20 to be moderate, and more than 20 to be large. Consequently, a change smaller than 5 points was considered to be no change. Taking into account both deteriorations and improvements, this results in a maximum of 7 subgroups.

\section{Step 3: Examining responsiveness}

Responsiveness to change was evaluated using a statistical indicator, the standardized response mean (SRM). The SRM is the change in score divided by the standard deviation of the change in score. It is independent of sample size and widely used today (Husted et al., 2000). SRMs were calculated for the EQ-5D Index and EQ VAS, for all subgroups of patients. Scores were interpreted using benchmarks for effect sizes: 0.20 through 0.49 was interpreted as small, 0.50 through 0.79 as moderate and $\geq 0.80$ as large (Cohen, 1988). Additionally, analysis of variance, with Games Howell post hoc procedures, was performed to compare the mean change scores on the EQ-5D Index and EQ VAS between the 'no change' subgroup and the other subgroups identified in step 2.

\section{RESULTS}

\section{Step 1. Selecting an anchor}

The global health scale of the EORTC QLQ-C30 correlated to the change scores of the EQ$5 D$ Index and EQ VAS ( $r=0.423$ and $r=0.634$ respectively). Hence, global health was found to be an appropriate anchor and was used to classify subgroups. 


\section{Step 2. Classifying patients into subgroups}

After twelve months, 6 patients (3\%) reported a large deterioration on global health, $17(9 \%)$ reported a moderate deterioration, $14(7 \%)$ reported a small deterioration, 55 (28\%) reported no change, $28(16 \%)$ reported a small improvement, 32 (17\%) a moderate improvement and 40 (21\%) reported a large improvement on global health.

Due to a relatively small number of patients reporting a moderate or large deterioration, it was decided to create one subgroup for patients with both moderate and large deteriorations ('moderate-large deterioration') and, for easy comparison, also one subgroup for both moderate and large improvements ('moderate-large improvement'). Hence, five subgroups were identified, classifying patients reporting a (1) moderate-large deterioration ( $n=23),(2)$ small deterioration $(n=14),(3)$ no change $(n=55),(4)$ a small improvement $(n=28)$ and $(5)$ moderate-large improvement in health status $(n=72)$.

\section{Step 3. Examining responsiveness}

Mean baseline scores, scores at the twelve month measurement and change scores are presented for all HRQoL measures in table 2. The EQ VAS and EQ-5D Index both moved in the expected direction, indicating negative changes (deterioration) in the subgroups reporting deterioration on global health of the EORTC QLQ-C30 and positive changes (improvements) in the subgroups reporting improvements on global health. Accordingly, only a minor change on the EQ VAS and no change on the EQ-5D Index were reported in the no change subgroup of the EORTC QLQ-C30.

Table 2. Baseline scores (T0), twelve months scores (T1) and mean change scores with standard deviations. SRMs of the EQ VAS and EQ-5D Index for all subgroups of patients.

\begin{tabular}{|c|c|c|c|c|c|c|c|c|c|c|c|}
\hline \multirow[b]{2}{*}{ Subgroup } & \multicolumn{3}{|c|}{ EORTC QLQ-C30 } & \multicolumn{4}{|c|}{ EQ-5D Index } & \multicolumn{4}{|c|}{ EQ VAS } \\
\hline & TO & $\mathrm{T} 1$ & $\Delta(\mathrm{sd})$ & TO & $\mathrm{T} 1$ & $\Delta(\mathrm{sd})$ & SRM & TO & $\mathrm{T} 1$ & $\Delta(\mathrm{sd})$ & SRM \\
\hline $\begin{array}{l}\text { Moderate-large } \\
\text { deterioration } \\
(n=23)\end{array}$ & 79.3 & 56.9 & $\begin{array}{l}-22.5 \\
(10.8)\end{array}$ & 73.0 & 59.8 & $\begin{array}{l}-13.2 \\
(11.2)\end{array}$ & -1.17 & 0.72 & 0.57 & $\begin{array}{l}-0.15 \\
(0.29)\end{array}$ & -0.52 \\
\hline $\begin{array}{l}\text { Small deteriora- } \\
\text { tion }(n=14)\end{array}$ & 75.6 & 67.3 & $\begin{array}{l}-8.3 \\
(0.0)\end{array}$ & 74.4 & 69.4 & $\begin{array}{l}-5.1 \\
(12.0)\end{array}$ & -0.42 & 0.73 & 0.72 & $\begin{array}{l}-0.01 \\
(0.18)\end{array}$ & -0.05 \\
\hline No change $(n=55)$ & 80.9 & 80.9 & $\begin{array}{l}0.0 \\
(0.0)\end{array}$ & 79.0 & 79.9 & $\begin{array}{l}0.7 \\
(8.8)\end{array}$ & 0.08 & 0.82 & 0.82 & $\begin{array}{l}0.00 \\
(0.21)\end{array}$ & 0.01 \\
\hline $\begin{array}{l}\text { Small improve- } \\
\text { ment }(n=28)\end{array}$ & 71.2 & 80.1 & $\begin{array}{l}8.3 \\
(0.0)\end{array}$ & 70.9 & 77.7 & $\begin{array}{l}6.1 \\
(7.7)\end{array}$ & 0.79 & 0.78 & 0.80 & $\begin{array}{l}0.02 \\
(0.14)\end{array}$ & 0.16 \\
\hline $\begin{array}{l}\text { Moderate-large } \\
\text { improvement } \\
(n=72)\end{array}$ & 58.2 & 85.6 & $\begin{array}{l}27.4 \\
(11.9)\end{array}$ & 65.0 & 77.4 & $\begin{array}{l}12.1 \\
(12.7)\end{array}$ & 0.95 & 0.71 & 0.83 & $\begin{array}{l}0.13 \\
(0.20)\end{array}$ & 0.62 \\
\hline
\end{tabular}

To examine responsiveness, SRMs were calculated for the EQ-5D Index and EQ VAS (table 2). In the subgroup of patients whose global health had not changed, accordingly, neither the SRM of the EQ-5D Index, nor of the EQ VAS indicated an effect. SRMs of the EQ-5D 
Index for the subgroups indicating a small deterioration or small improvement were too small (i.e. SRM < 0.20) to be considered as an effect. In contrast, SRMs of the EQ VAS indicated a small effect in these subgroups. SRMs of the subgroups with moderate and large improvements or deteriorations in global health indicated a moderate effect on the EQ-5D Index (i.e. SRM > 0.50) and a large effect on the EQ VAS (i.e. SRM > 0.80).

Analysis of variance procedures were performed to evaluate whether the EQ-5D could discriminate between the five subgroups (table 3). Results indicated that when the EQ$5 \mathrm{D}$ Index score was used as the outcome measure, the subgroup reporting no change on global health differed significantly from the subgroup reporting moderate and large improvements. The subgroups reporting small improvements or a small or moderate and large deterioration could not be differentiated from the 'no change' subgroup. The EQ VAS on the other hand was able to discriminate between the 'no change' subgroup and the subgroups reporting a moderate and large improvement and moderate and large deterioration.

Table 3. Analysis of variance

\begin{tabular}{|c|c|c|c|c|c|}
\hline \multicolumn{2}{|c|}{ Global health EORTC QLQ-C30 } & \multicolumn{2}{|l|}{ EQ-5D Index } & \multicolumn{2}{|l|}{ EQ VAS } \\
\hline & Subgroup & $\begin{array}{l}\text { Mean difference } \\
\text { (SE) }\end{array}$ & p-value & $\begin{array}{l}\text { Mean difference } \\
\text { (SE) }\end{array}$ & p-value \\
\hline \multirow{4}{*}{$\begin{array}{l}\text { No change } \\
(n=55)\end{array}$} & $\begin{array}{l}\text { Moderate-large } \\
\text { deterioration }(n=23)\end{array}$ & $-0.14(0.07)$ & 0.228 & $-13.88(2.65)$ & $<0.001$ \\
\hline & $\begin{array}{l}\text { Small deterioration } \\
(n=14)\end{array}$ & $0.01(0.05)$ & 1.000 & $-5.78(3.44)$ & 0.470 \\
\hline & $\begin{array}{l}\text { Small improvement } \\
(n=28)\end{array}$ & $0.02(0.04)$ & 0.984 & 5.37 (1.93) & 0.054 \\
\hline & $\begin{array}{l}\text { Moderate-large } \\
\text { improvement }(n=72)\end{array}$ & $0.13(0.04)$ & 0.006 & $11.38(1.97)$ & $<0.001$ \\
\hline
\end{tabular}

\section{DISCUSSION}

An increasing number of clinical trials is investigating the effectiveness of follow-up strategies and psychosocial interventions for breast cancer patients after treatment, using HRQoL as an important outcome measure (Goodwin et al., 2003; Montazeri et al., 2008). Hence, a good responsiveness of the HRQoL measure used seems essential. Our study showed that the EQ-5D was able to detect both improvements and deteriorations in health. However, according to Cohen's benchmarks for effect sizes (Cohen, 1988), the EQ$5 D$ Index was not responsive to small changes in health. The inability of the EQ-5D Index to detect small changes might be explained by its structure. It is generally acknowledged that more response options lead to a higher responsiveness (Wiebe et al., 2003). The domains of the EQ-5D have only three response levels, making it difficult to pick up small 
changes in health. In addition, in the subgroup of patients reporting no change and the subgroup reporting a small improvement on global health, baseline scores on the EQ$5 \mathrm{D}$ Index were relatively high. These high scores were a result of large proportions of respondents already in the top category of domains of the EQ-5D. This ceiling effect is a well known feature of the EQ-5D and left little room for improvement (Brazier et al., 2004). A straightforward solution would be to attempt to produce a better, more responsive, generic index measure. Recent studies on an EQ-5D with five response levels for each domain showed increased descriptive power and suggest better discriminatory power (Janssen et al., 2008a; Janssen et al., 2008b). Hence a less severe ceiling effect and increased benefit in the detection of small health changes are expected (Janssen et al., 2008b). Unfortunately, an official five-level descriptive system is not yet available.

Additional analysis of variance procedures to investigate responsiveness showed that the EQ-5D Index and the EQ VAS both could not differentiate between subgroups reporting no change and small changes in global health. For the EQ-5D Index this was in accordance with the small SRMs in these subgroups. For the EQ VAS however, the non-significant differences were unexpected, as the SRMs indicated moderate effects. This inability of the EQ VAS to discriminate might be explained by the small number of patients in these subgroups ( $n=14$ and $n=28$ respectively). Analysis of variance procedures and especially post hoc procedures are sensitive to population variances and differences in sample size in subgroups. Hence, with a larger sample size, the EQ VAS might have been able to differentiate between subgroups with no change and small changes in health. This argument also holds true for the EQ-5D Index, which could not discriminate between the 'no change' subgroup and the subgroup reporting a moderate-large deterioration in health $(n=23)$.

A limitation of this study was that the responsiveness was investigated using a single anchor, while ideally multiple anchors should be used to investigate the responsiveness of an instrument (Guyatt et al., 2002). A clinical variable, such as whether or not a recurrence was detected, would be a suitable second anchor to classify subgroups of patients. However, in the clinical trial from which participants were used for these analyses, only few $(<10)$ recurrences were reported, and unfortunately, these participants were study drop-outs. Hence, an appropriate second anchor was not available. Further research into the responsiveness of the EQ-5D in breast cancer patients should aim to include multiple anchors.

In summary, results of this study showed that the EQ-5D was able to capture both improvements and deteriorations in HRQoL of breast cancer patients after treatment, but small changes in health were not recognized as being meaningful. However, in economic evaluations the EQ-5D is primarily used to measure outcome for QALY analysis rather than measuring HRQoL for clinical purposes. Within the framework of economic evaluations, an incremental cost-effectiveness ratio (i.e. additional cost per QALY gained) is more informative than the difference in HRQoL alone. Therefore, a small difference in the EQ- 
$5 \mathrm{D}$ Index might still be meaningful if additional costs for such a change in HRQoL are very low. Hence, the EQ-5D should indeed be able to pick up relevant changes in health and should be able to differentiate between subgroups of patients to some extent, but cut-off points for effect sizes or discriminative ability are less relevant in the context of economic evaluations.

\section{CONCLUSION}

In this study the responsiveness of the EQ-5D was investigated for its use in economic evaluations of health interventions in breast cancer patients after primary treatment. The EQ-5D was able to detect improvements and deteriorations in health and could discriminate between patients with no change in health and patients with moderatelarge changes in health. Therefore, the EQ-5D seems an appropriate HRQoL measure for economic evaluations in breast cancer patients after treatment. 


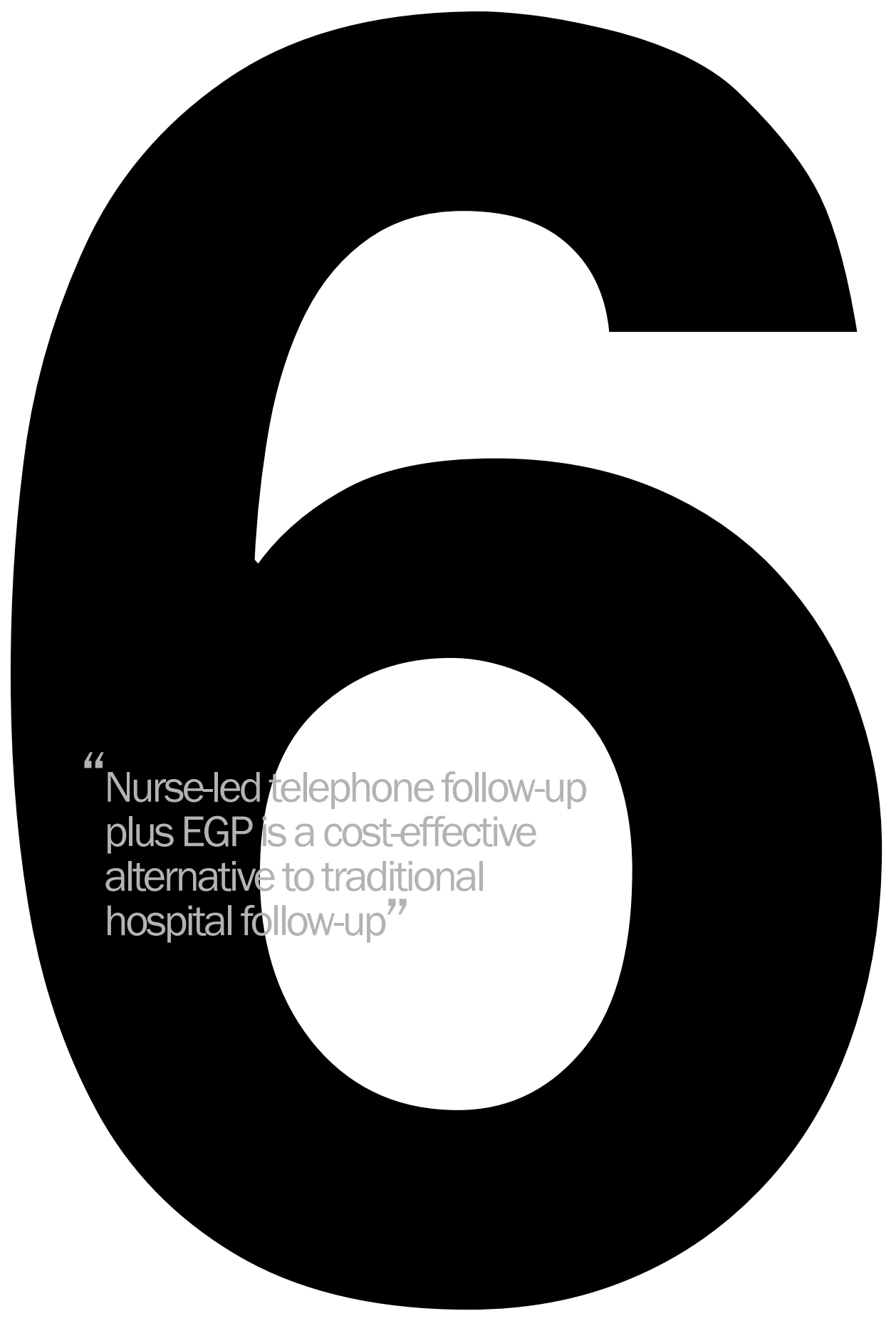




\title{
Economic evaluation of four follow-up strategies after curative treatment for breast cancer: results of an RCT
}

KIMMAN ML, DIRKSEN CD, VOOGD AC, FALGER P, GIJSEN BCM, THURING M, LENSSEN A, VAN DER ENT F, VERKEYN J, HAEKENS C, HUPPERETS P, NUYTINCK JKS, VAN RIET Y, BRENNINKMEIJER SJ, SCHEIJMANS L, KESSELS A, LAMBIN Ph, BOERSMA L.

UNDER REVIEW

\begin{abstract}
[Objective] An economic evaluation of four follow-up strategies for the first year after breast cancer treatment, including traditional hospital follow-up, nurse-led telephone follow-up, and the former strategies combined with an educational group program (EGP).

[Design] Cost-effectiveness analysis at 12 months follow-up alongside a randomized controlled trial (RCT). [Setting] Multicenter study in the Netherlands. [Participants] 299 breast cancer patients from seven hospitals and two radiotherapy clinics in the Netherlands who were treated with curative intent. [Interventions] Participants were randomized to follow-up care as usual (3-monthly outpatient clinic visits), nurse-led telephone follow-up, or the former strategies combined with the EGP. [Main outcome measures] Costs were measured using individual level cost data from cost diaries and hospital registrations. Quality-adjusted life years (QALYs) were measured using the EQ5D. Outcomes were expressed in incremental cost-effectiveness ratios (ICERs) and costeffectiveness acceptability curves. [Results] Hospital follow-up plus EGP yielded most QALYs (0.776; 95\% Cl 0.753 to 0.799), but also incurred the highest mean annual costs (€4914; 95\% Cl 3793 to 6192). The ICER of hospital follow-up plus EGP versus the next best alternative, nurse-led telephone follow-up plus EGP (0.772 QALYs; 95\% Cl 0.745 to 0.797 and €3971; 95\% Cl 2975 to 5186), amounted to €235.750/QALY. As hospital and telephone follow-up without EGP both incurred higher costs and less QALYs than telephone follow-up plus EGP, the former strategies were judged inferior. Assuming a threshold value of $€ 40.000$ per QALY, hospital follow-up plus EGP was not considered cost-effective and therefore telephone follow-up plus EGP was the preferred strategy. The probability of telephone follow-up plus EGP being cost-effective ranged from 49\% to $62 \%$ for different QALY threshold values. Secondary and sensitivity analyses showed that these results were robust. [Conclusions] Nurse-led telephone follow-up plus EGP is likely to be a cost-effective follow-up strategy after treatment for breast cancer. It may lead to substantial cost reductions, without loss of health-related quality of life compared to traditional hospital follow-up. It seems warranted to critically assess current guidelines for breast cancer follow-up.
\end{abstract}




\section{INTRODUCTION}

In the Netherlands, one out of eight women will be diagnosed with breast cancer at some moment in her life (Kiemeney et al., 2008). After treatment, these women will bring frequent follow-up visits to the hospital in order to be examined for local disease recurrence or second primary breast cancer, and for information and psychosocial support (Pestalozzi \& Castiglione, 2008; Struikmans et al., 2008). However, the need for frequent follow-up of breast cancer patients remains controversial despite almost two decades of research. There has been much debate regarding the effectiveness of routine follow-up of these patients (Kimman et al., 2007a; Montgomery et al., 2007; Pennery \& Mallet, 2000), while at the same time it is associated with a significant cost burden (Meropol et al., 2009). Less specialized follow-up strategies have been proposed demonstrating equal patient satisfaction and health-related quality of life (HRQoL) compared to traditional hospital visits (Grunfeld et al., 2006, Koinberg et al., 2004, Beaver et al., 2009b, Montgomery et al., 2008a). To date, these alternative strategies, however, have not been structurally implemented in clinical practice. Several authors have urged to evaluate the costs in relation to the benefits of current and alternative follow-up strategies (Grunfeld, 2009; Meropol et al., 2009; Montgomery et al., 2007). Therefore, we performed a randomized controlled trial investigating the effectiveness of both a nurse-led telephone follow-up instead of hospital visits and a short educational group program (EGP) during the first year after breast cancer treatment, which included an economic evaluation (Kimman et al., 2007 b). Results of this economic evaluation may improve resource-allocation decisions and enhance dissemination of cost-effective follow-up strategies.

\section{METHODS}

\section{Design, sample and interventions}

Details of the trial design and protocol execution have been reported previously (ISRCTN 74071417) (Kimman et al., 2007b). In sum, between 2005 and 2008, 320 females were recruited through seven hospitals and two radiotherapy clinics in the South of the Netherlands. Participants were eligible when they had completed treatment for breast cancer (tumor stage I-III) within six weeks prior to randomization. They were randomly assigned to one of four follow-up strategies (study arms) for the first 18 months following treatment; i.e. 1) hospital follow-up as usual: five outpatient clinic visits in the first 18 months (at 3, 6, 9, 12 and 18 months), including a mammography at 12 months; 2) nurseled telephone follow-up: a mammography at 12 months combined with an outpatient clinic visit, and telephone interviews at the same time points as for usual follow-up; 3 ) arm 1 plus educational group program (EGP); 4) arm 2 plus EGP. Telephone follow-up was performed by a trained breast care nurse $(\mathrm{BCN})$ and consisted of a semi-structured questionnaire including screening for physical and psychological symptoms, treatment side effects, compliance with hormonal therapy, and an open discussion of these issues. The EGP consisted of two interactive group sessions of 2.5 hours each: a BCN provided 
information on possible treatment side effects and topics such as prostheses, fatigue, and signs and symptoms of possible recurrences. A health care psychologist addressed psychological and social consequences of breast cancer, and discussed possible psychological coping strategies.

The economic evaluation compared costs and effects of these four follow-up strategies from a societal perspective, with a time horizon of one year. During the trial 21 patients (6.5\%) dropped out for various reasons, as reported in a previous paper (Kimman et al., 2010a). Hence, data for the economic evaluation was available of 299 patients. The mean age of the sample was 55.8 years (range 27 to 78). Details on tumor stage and received treatment of participants are provided in table 1.

Table 1. Baseline characteristics of participants of the economic evaluation $(n=299)$. Values are numbers (percentages) unless stated otherwise.

\begin{tabular}{ll} 
& All participants $(\mathbf{n}=\mathbf{2 9 9})$ \\
Age at recruitment in years & \\
Mean (SD) & $55.8(9.9)$ \\
Range & $23-78$ \\
Level of education & \\
Low & $102(34.1)$ \\
Middle & $118(39.5)$ \\
High & $79(26.4)$ \\
Employment status & \\
Paid employment & $91(30.4)$ \\
Hours of paid employment per week (mean and SD) & $8.9(13.6)$ \\
Tumor stage & \\
Stage I & $181(60.5)$ \\
Stage Ila & $69(23.1)$ \\
Stage IIb & $21(7.0)$ \\
Stage III & $24(8.0)$ \\
Unknown & $4(1.3)$ \\
Treatment modality & \\
Surgery & $29(9.7)$ \\
Surgery + radiotherapy (RT) & $178(59.5)$ \\
Surgery + chemotherapy (CH) & $15(5.0)$ \\
Surgery + RT + CH & $77(25.8)$ \\
Hormonal therapy (yes) & $94(31.4)$ \\
\hline
\end{tabular}

\section{Measure of effect}

The quality-adjusted life year (QALY) was chosen to represent health gain (Earnshaw \& Lewis, 2008). The QALY is a measure of life expectancy weighted by health-related quality of life (HRQoL), the latter which is represented by utility scores. In the present study, HRQoL was measured with the EQ-5D, a questionnaire responsive to changes in health in breast cancer patients after conclusion of treatment (Kimman et al., 2009). The EQ-5D comprises five dimensions: mobility, self-care, usual activities, pain/discomfort and anxiety/depression. Each dimension is rated at three levels: no problems (1), some 
problems (2) and major problems (3). Based on preferences elicited from a general UK population, EQ-5D health states (e.g. 1-1-2-1-3) may be converted into utility scores (Index scores) (Dolan, 1997). In the present study, utility scores were measured at baseline, and 3, 6 and 12 months after treatment and were subsequently used to calculate QALYs according to the following formula: $\{[$ (utility score at baseline + utility score at 3 months $) / 2] * 3 / 12+[$ (utility score at 3 months + utility score at 6 months $) / 2] * 3 / 12+$ [(utility score at 6 months + utility score at 12 months $) / 2] * 6 / 12$ \}.

\section{Measure of costs}

Cost analysis was performed from the societal perspective which includes health care and non health care related costs (e.g. productivity loss, informal care) as a result of breast cancer (Earnshaw \& Lewis, 2008; Oostenbrink et al., 2004). Hormonal therapy costs were not included since these were considered part of standard treatment if feasible. Complete hospital resource use data on an individual level (e.g. diagnostic procedures, outpatient clinic visits, telephone interviews) were retrieved from hospital information systems. Resource use outside the hospital (e.g. general practitioner visits, physical rehabilitation) was determined by means of cost diaries as kept by participants (Goossens et al., 2000). These were completed prospectively at 3, 6 and 12 months, for four week periods each. Resource use was interpolated to the study period under the assumption that data obtained from these cost diaries would be representative of the in between periods (Goossens et al., 2000).

Cost prices for resource use were primarily obtained from the Dutch governmental manual for health care cost analysis (Oostenbrink et al., 2004). All cost prices were converted to 2008 Euros by means of price index numbers (Bureau of Statistics, 2008). Cost prices per unit of resource use for the main cost drivers are presented in table 2 (page 62). Costs for hospital visits were based on cost prices for academic hospitals. Productivity costs were calculated using the human capital approach (HCA) (Hodgson, 1994). Costs for the EGP included the hourly wages of the health care psychologist and BCN, the use of facilities and the information booklet, as well as travel costs of patients. The cost price for telephone follow-up consisted of the average duration of the follow-up (18 minutes) multiplied by the hourly wage of a specialized nurse, adding $45 \%$ for hospital overhead costs and the average costs of a telephone call.

\section{Statistical analysis}

The base case analysis was performed based on intention to treat. Multiple imputation was used to replace missing values with plausible estimates, and generated five data sets (Schafer, 1999). Results were provided as pooled estimates of these sets. Bootstrap simulations with 1000 replications were used to calculate 95\% confidence intervals around mean costs and effects of the four study arms (Briggs et al., 1997). Differences between the four groups in resource use, costs and QALYs were reported descriptively and were not compared statistically to avoid problems with multiple testing, and because the 
focus of the economic evaluation was on the combined costs and effects in order to assess cost-effectiveness. To investigate cost-effectiveness of the four strategies, incremental cost-effectiveness ratios (ICERs) were calculated. When more than two strategies are compared, as is the case in this study, ICERs are calculated using rules of dominance and extended dominance (Fenwick \& Byford, 2005). In this approach, strategies are ranked by QALYs, from the most effective to the least effective, and if a strategy is less effective and more costly than the previous strategy, it is said to be dominated and is excluded from the calculation of ICERs. Hence, this process compares strategies in terms of observed differences in costs and effects, regardless of the statistical significance of the difference. Bootstrapped sample means (1000) from costs and effectiveness data were used to quantify the uncertainty associated with the ICERs. Subsequently, cost-effectiveness acceptability curves (CEACs) were derived in order to show the probability of each strategy being the optimal choice, for a range of possible maximum values a decision maker is willing to pay for a QALY (Fenwick et al., 2004). Bootstrapping was performed using Excel 2000. Other analyses were performed using the SPSS package, version $17.0^{\circledR}$ for Windows (SPSS INC 2009)

\section{Secondary analyses}

Secondary analyses were performed to assess the robustness of the base case results. First, an analysis was performed according to the actually applied follow-up strategy (per protocol analysis). Second, an analysis was performed excluding productivity costs. A third analysis took a limited health care perspective, including only health care related costs. Several one-way sensitivity analyses were performed in order to assess the impact of the unit prices of telephone contacts and hospital visits. We used the highest reported value for telephone contact (55 minutes) and the generic cost price for a hospital visit (€54.15). A series of subgroup analyses compared cost-effectiveness data according to age, use of chemotherapy, and levels of anxiety and support from partner. Finally, a subsample of patients for whom 18 months data were available $(n=244)$ was used in order to investigate whether cost-effectiveness results could be extrapolated to the $2^{\text {nd }}$ year after treatment. In this analysis, costs and effects incurred between 12 and 18 months were discounted using a 4\% discount rate (Oostenbrink et al., 2004).

\section{RESULTS}

\section{Effects and costs}

Table 3 summarizes the percentage of patients reporting any problems (level (2) and (3) combined) on the five dimensions of the EQ-5D as well as utility (index) scores, at all measurement time points. Due to substantial baseline utility differences, subsequent utility scores are (also) presented using a regression-based adjustment as recommended by Manca et al. (2005), and taking into account regression to the mean effects (van Asselt et al., 2009). Utility gains were most notable in the telephone 
Table 2. Unit prices. All unit prices are expressed in Euros at 2008 values. EGP = educational group program, $\mathrm{MS}=$ medical specialist, $\mathrm{BCN}=$ breast care nurse.

\begin{tabular}{|c|c|}
\hline Cost category & Unit price in Euro (2008) ${ }^{a, b}$ \\
\hline \multicolumn{2}{|l|}{ Direct health care costs } \\
\hline General practitioner & 21.88 / visit ${ }^{b 1}$ \\
\hline Physiotherapist & 24.64 / visit ${ }^{b 1}$ \\
\hline Rehabilitation program $^{c}$ & $1500^{\mathrm{b} 2}$ \\
\hline Educational group program (EGP) ${ }^{d}$ & $135^{\mathrm{b} 3}$ \\
\hline Other health care professionals ${ }^{e}$ & 24.64 / visit ${ }^{b 1}$ \\
\hline Home care domestic & $23.50 /$ hour $^{\mathrm{b} 1}$ \\
\hline Home care nursing & 43.75 / hour b1 \\
\hline Medication $^{f}$ & Various ${ }^{\mathrm{b} 4}$ \\
\hline Hospital follow-up (MS / BCN) & 108.30 / visit ${ }^{b 1}$ \\
\hline Telephone MS & 12.83 / 5 min call b5 \\
\hline Telephone BCN & 21.50 / follow-up contact ${ }^{\text {b5 }}$ \\
\hline Postoperative surgeryg & Various / procedure ${ }^{\mathrm{b} 1}$ \\
\hline Mammography/ultrasound & $79.75 / 82.10 /$ test $^{\mathrm{b} 1}$ \\
\hline Breast biopsy & 127.19 / procedure ${ }^{\mathrm{b} 1}$ \\
\hline Laboratory tests & Various / test ${ }^{\mathrm{b} 1}$ \\
\hline Cardiology or lung tests & Various / test ${ }^{\mathrm{b} 1}$ \\
\hline Other diagnostics ${ }^{\mathrm{h}}$ & Various / procedure ${ }^{\mathrm{b} 1}$ \\
\hline Costs for in-hospital days ${ }^{i}$ & $598.89 /$ day $^{\mathrm{b} 1}$ \\
\hline \multicolumn{2}{|l|}{ Direct non health care costs } \\
\hline Paid help & $10 /$ hour $^{\mathrm{b} 1}$ \\
\hline Informal care & 8.99 / hour b1 \\
\hline \multicolumn{2}{|l|}{ Costs of lost production } \\
\hline Paid work & 19.22 / hour b6 \\
\hline Domestic tasks & 8.99 / hour b1 \\
\hline \multicolumn{2}{|l|}{ Out of pocket costs } \\
\hline Reported by patients ${ }^{j}$ & Various ${ }^{\mathrm{b} 7}$ \\
\hline Drugs $^{j}$ & Various ${ }^{\mathrm{b} 7}$ \\
\hline
\end{tabular}

a When necessary, cost prices were converted to 2008 by means of price index numbers.

b Source of unit price: ${ }^{1}$ Dutch manual for cost prices (Oostenbrink et al 2004); ${ }^{2}$ Tariff for health insurer; ${ }^{3}$ Calculation included cost location, wage of health care providers, material, travel costs; ${ }^{4}$ www. farmacotherapeutischkompas.nl (including tax + recepy fee of $€ 6.11$ ); ${ }^{5}$ Calculations based on time per call multiplied by wage and overhead costs; ${ }^{6}$ Gross wage, female $55-65$, CBS; ${ }^{7}$ as reported by patient.

${ }^{c}$ Rehabilitation program: a 12-week group program for cancer patients combining physical exercise and psycho-education.

${ }^{\mathrm{d}}$ Educational group program (study intervention): two group sessions outside hospital by a health care psychologist and breast care nurse (including booklet).

e Other health care professionals such as lymph edema therapist, alternative healers.

${ }^{f}$ Medication was sub grouped to tranquilizers, antibiotics, antidepressants, pain killers, drugs for sideeffects of treatment, and other drugs.

gIncluded breast reconstruction, treatment of wounds/abscess, lymph edema.

${ }^{\mathrm{h}}$ Included CTs, MRIs, PET whole body scans, et cetera

${ }^{i}$ Average of total costs for in-hospital days, including intake assessment and nursing.

j Out-of-pocket costs included costs for over the counter medication, massage, parking costs, et cetera 
Table 3. Percentage of patients reporting ANY problems on the EQ-5D, the EQ-5D index scores at baseline, 3, 6 and 12 months after treatment and adjusted QALYs for the four study arms.

\begin{tabular}{|c|c|c|c|c|c|}
\hline Measurement & Dimensions of EQ-5D & $\begin{array}{l}\text { Hospital } \\
\text { f-up }\end{array}$ & $\begin{array}{l}\text { Telephone } \\
\text { f-up }\end{array}$ & $\begin{array}{l}\text { Hospital } \\
\text { f-up + EGP }\end{array}$ & $\begin{array}{l}\text { Telephone } \\
\text { f-up + EGP }\end{array}$ \\
\hline \multicolumn{6}{|c|}{$\%$ of patients reporting ANY problems } \\
\hline \multirow[t]{6}{*}{ Baseline } & Mobility & 22.2 & 30.3 & 16.0 & 22.2 \\
\hline & Self-care & 5.4 & 5.3 & 2.7 & 3.0 \\
\hline & Usual activities & 52.7 & 54.7 & 48.0 & 55.4 \\
\hline & Pain/discomfort & 64.9 & 68.4 & 50.7 & 66.2 \\
\hline & Anxiety/depression & 42.7 & 50.0 & 33.3 & 36.5 \\
\hline & Index score & 0.736 & 0.728 & 0.804 & 0.722 \\
\hline \multirow[t]{7}{*}{3 months } & Mobility & 21.1 & 30.8 & 16.8 & 24.9 \\
\hline & Self-care & 5.4 & 3.7 & 1.3 & 5.7 \\
\hline & Usual activities & 47.0 & 46.8 & 42.7 & 46.8 \\
\hline & Pain/discomfort & 63.8 & 60.0 & 42.1 & 51.4 \\
\hline & Anxiety/depression & 51.1 & 47.4 & 37.9 & 41.9 \\
\hline & Index score & 0.721 & 0.757 & 0.810 & 0.775 \\
\hline & Index score (adjusted) ${ }^{1}$ & 0.727 & 0.767 & 0.780 & 0.789 \\
\hline \multirow[t]{7}{*}{6 months } & Mobility & 21.6 & 26.3 & 18.1 & 26.4 \\
\hline & Self-care & 4.3 & 0.0 & 0.5 & 8.6 \\
\hline & Usual activities & 36.5 & 40.0 & 33.3 & 47.0 \\
\hline & Pain/discomfort & 54.6 & 53.4 & 45.9 & 54.6 \\
\hline & Anxiety/depression & 38.6 & 45.8 & 40.0 & 43.5 \\
\hline & Index score & 0.760 & 0.771 & 0.803 & 0.758 \\
\hline & Index score (adjusted) ${ }^{1}$ & 0.766 & 0.780 & 0.776 & 0.770 \\
\hline \multirow[t]{7}{*}{12 months } & Mobility & 24.9 & 18.2 & 26.7 & 28.4 \\
\hline & Self-care & 10.0 & 5.3 & 2.4 & 10.5 \\
\hline & Usual activities & 34.6 & 37.4 & 36.0 & 33.0 \\
\hline & Pain/discomfort & 60.8 & 57.6 & 48.0 & 50.0 \\
\hline & Anxiety/depression & 34.1 & 40.3 & 36.3 & 33.2 \\
\hline & Index score & 0.733 & 0.768 & 0.786 & 0.771 \\
\hline & Index score (adjusted) ${ }^{1}$ & 0.739 & 0.777 & 0.759 & 0.783 \\
\hline QALY & & 0.740 & 0.762 & 0.801 & 0.761 \\
\hline QALY (adjusted) ${ }^{1}$ & & 0.747 & 0.769 & 0.776 & 0.772 \\
\hline $95 \% \mathrm{Cl}$ & & $(0.707-0.778)$ & $(0.746-0.794)$ & (0.753-0.799) & $(0.745-0.797)$ \\
\hline
\end{tabular}

\footnotetext{
${ }^{1}$ QALYs were adjusted using a regression-based approach
} 
follow-up and telephone follow-up plus EGP groups, but overall QALYs were highest for hospital follow-up plus EGP. This is partly explained by the high baseline utility scores. Adjustment for baseline differences resulted in smaller QALY differences, but hospital follow-up plus EGP remained the most effective strategy. We used the adjusted QALY for the construction of cost-effectiveness acceptability curves, which reflects the finding that baseline scores were higher for this strategy. Table 4 and 5 show resource utilization and costs during the study period.

Table 4. Mean resource use per patient over 12 months (in number of contacts or tests unless stated otherwise). $\mathrm{EGP}=$ Educational group program, $\mathrm{MS}=$ medical specialist, $\mathrm{BCN}=$ breast care nurse

\begin{tabular}{lllll}
\hline Resource category & $\begin{array}{l}\text { Hospital f-up } \\
\text { mean (SD) }\end{array}$ & $\begin{array}{l}\text { Telephone f-up } \\
\text { mean (SD) }\end{array}$ & $\begin{array}{l}\text { Hosp + EGP } \\
\text { mean (SD) }\end{array}$ & $\begin{array}{l}\text { Tel + EGP } \\
\text { mean (SD) }\end{array}$ \\
\hline $\begin{array}{l}\text { Direct health care resources } \\
\text { General practitioner }\end{array}$ & $1.7(2.7)$ & $1.6(3.1)$ & $1.5(2.8)$ & $0.8(1.7)$ \\
Physiotherapist & $7.0(14.5)$ & $8.6(16.2)$ & $10.1(19.8)$ & $8.5(16.0)$ \\
Revalidation & $0.1(0.3)$ & $0.2(0.4)$ & $0.1(0.3)$ & $0.2(0.4)$ \\
Other health care professionals & $1.5(3.4)$ & $3.0(7.2)$ & $2.1(3.6)$ & $2.1(3.7)$ \\
Home care domestic (in hours) & $8.9(32.3)$ & $7.3(27.8)$ & $6.2(27.1)$ & $3.7(11.7)$ \\
Home care nursing (in hours) & $0.4(1.4)$ & $1.1(5.2)$ & $0.4(1.5)$ & $0.6(2.0)$ \\
Visits MS & $5.5(2.4)$ & $2.6(1.8)$ & $5.3(2.0)$ & $2.9(2.4)$ \\
Visits BCN & $0.5(1.3)$ & $0.7(1.4)$ & $0.5(1.4)$ & $0.6(1.2)$ \\
Telephone MS & $0.2(0.5)$ & $0.1(0.6)$ & $0.2(0.8)$ & $0.1(0.3)$ \\
Telephone BCN & $0.1(0.5)$ & $2.4(1.2)$ & $0.1(0.3)$ & $2.3(1.0)$ \\
Mammography/ultrasound & $1.6(1.1)$ & $1.7(1.0)$ & $1.5(0.8)$ & $1.7(1.0)$ \\
Biopsy & $0.1(0.3)$ & $0.1(0.4)$ & $0.1(0.3)$ & $0.1(0.4)$ \\
Laboratory tests & $11.8(23.9)$ & $5.7(16.0)$ & $4.6(8.8)$ & $4.4(13.7)$ \\
Cardiology and lung tests & $0.3(1.1)$ & $0.0(0.1)$ & $0.1(0.4)$ & $0.1(0.2)$ \\
Other diagnostic tests & $1.2(2.1)$ & $0.6(1.2)$ & $0.9(1.6)$ & $0.5(0.9)$ \\
In-hospital days & $0.6(2.5)$ & $0.1(0.4)$ & $0.1(0.9)$ & $0.1(0.3)$ \\
Direct non health care resources \\
Paid help (in hours) & $18.4(50.7)$ & $6.9(26.4)$ & $16.5(37.8)$ & $7.7(23.9)$ \\
Informal care (in hours) & $21.7(65.9)$ & $45.8(136.6)$ & $19.7(38.5)$ & $37.1(125.9)$ \\
Productivity loss & & & & \\
Paid work (in hours) & $60.3(151.3)$ & $85.2(228.4)$ & $98.0(238.9)$ & $68.9(188.5)$ \\
Domestic tasks (in hours) & $32.7(105.1)$ & $45.9(123.4)$ & $25.5(77.8)$ & $14.7(27.9)$ \\
\hline & & & & \\
\hline
\end{tabular}


Table 5. Mean costs per patient (in Euros) over 12 months.

$\mathrm{EGP}=$ educational group program, $\mathrm{MS}=$ medical specialist, $\mathrm{BCN}=$ breast care nurse

\begin{tabular}{|c|c|c|c|c|}
\hline \multirow[b]{2}{*}{ Cost category } & \multicolumn{4}{|c|}{ Mean costs in 12 months (Euros) } \\
\hline & Hospital f-up & $\begin{array}{l}\text { Telephone } \\
\text { f-up }\end{array}$ & $\begin{array}{l}\text { Hospital f-up } \\
\text { + EGP }\end{array}$ & $\begin{array}{l}\text { Telephone } \\
\text { f-up + EGP }\end{array}$ \\
\hline \multicolumn{5}{|l|}{ Direct health care costs } \\
\hline General practitioner & 81 & 77 & 63 & 50 \\
\hline Physiotherapist & 172 & 212 & 249 & 209 \\
\hline Revalidation & 114 & 237 & 108 & 219 \\
\hline Educational group program & 0 & 0 & 135 & 135 \\
\hline Other health care professionals & 218 & 305 & 303 & 318 \\
\hline Home care (domestic/nursing) & 226 & 219 & 166 & 111 \\
\hline Medication & 23 & 20 & 18 & 23 \\
\hline Visits MS & 712 & 357 & 666 & 350 \\
\hline Visits BCN & 59 & 83 & 57 & 67 \\
\hline Telephone MS & 3 & 3 & 2 & 1 \\
\hline Telephone BCN & 2 & 52 & 2 & 49 \\
\hline Postoperative surgery & 83 & 55 & 42 & 0 \\
\hline Mammography/ultrasound & 131 & 137 & 123 & 139 \\
\hline Breast biopsy & 9 & 12 & 14 & 10 \\
\hline Laboratory tests & 92 & 47 & 69 & 41 \\
\hline Cardiology or lung tests & 12 & 0 & 2 & 1 \\
\hline Other diagnostic tests & 148 & 69 & 135 & 65 \\
\hline Costs for in-hospital days & 362 & 53 & 82 & 43 \\
\hline Subtotal health care costs & 2447 & 1938 & 2236 & 1831 \\
\hline (Bootstrapped 95\% Cl) & $(1974-3014)$ & (1604 - 2303) & $(1864-2713)$ & $(1468-2196)$ \\
\hline \multicolumn{5}{|l|}{ Direct non health care costs } \\
\hline Paid help & 184 & 69 & 165 & 77 \\
\hline Informal care & 195 & 412 & 177 & 333 \\
\hline Subtotal & 379 & 481 & 342 & 410 \\
\hline (Bootstrapped 95\% Cl) & $(196-609)$ & $(256-807)$ & $(232-473)$ & $(201-682)$ \\
\hline \multicolumn{5}{|l|}{ Costs of lost production } \\
\hline Paid work & 1159 & 1637 & 1884 & 1325 \\
\hline Domestic tasks & 294 & 412 & 230 & 133 \\
\hline Subtotal & 1453 & 2049 & 2114 & 1458 \\
\hline (Bootstrapped 95\% Cl) & $(848-2163)$ & $(1159-3111)$ & $(1202-3321)$ & $(692-2344)$ \\
\hline \multicolumn{5}{|l|}{ Out of pocket costs } \\
\hline Reported by patients & 130 & 177 & 202 & 257 \\
\hline Drugs & 10 & 27 & 20 & 15 \\
\hline Subtotal & 140 & 204 & 222 & 272 \\
\hline (Bootstrapped 95\% Cl) & $(89-209)$ & $(141-277)$ & $(133-340)$ & $(144-417)$ \\
\hline Subtotal non health care costs & 1972 & 2734 & 2678 & 2140 \\
\hline (Bootstrapped 95\% Cl) & $(1308-2713)$ & $(1706-3873)$ & $(1642$ - 3941) & $(1171-3317)$ \\
\hline TOTAL COSTS & 4419 & 4672 & 4914 & 3971 \\
\hline (Bootstrapped 95\% Cl) & $(3410-5501)$ & (3489 - 6033) & $(3793-6192)$ & $(2975-5186)$ \\
\hline
\end{tabular}




\section{Economic analysis}

In the base case analysis, hospital follow-up plus EGP yielded most QALYs (0.776), but also incurred highest mean annual costs (€4914). The ICER of hospital follow-up plus EGP versus the next best alternative, telephone follow-up plus EGP (0.772 QALYs and $€ 3971$ ), amounted to €235.750/QALY. Assuming a threshold of $€ 40.000$, as is argued for by the Dutch Council for Public Health and Health Care (RVZ, 2006), hospital follow-up plus EGP is not considered cost-effective and therefore telephone follow-up plus EGP was the preferred strategy (table 6). As hospital and telephone follow-up without EGP both incurred higher costs and less QALYs than telephone follow-up plus EGP, the former were judged to be dominated (i.e. inferior).

Figure 1 shows the cost-effectiveness acceptability curves and indicates that for a range of QALY threshold values ( $€ 0$ - $€ 80.000$ ), the probability that telephone follow-up plus EGP was cost-effective ranged between 49 and $62 \%$.

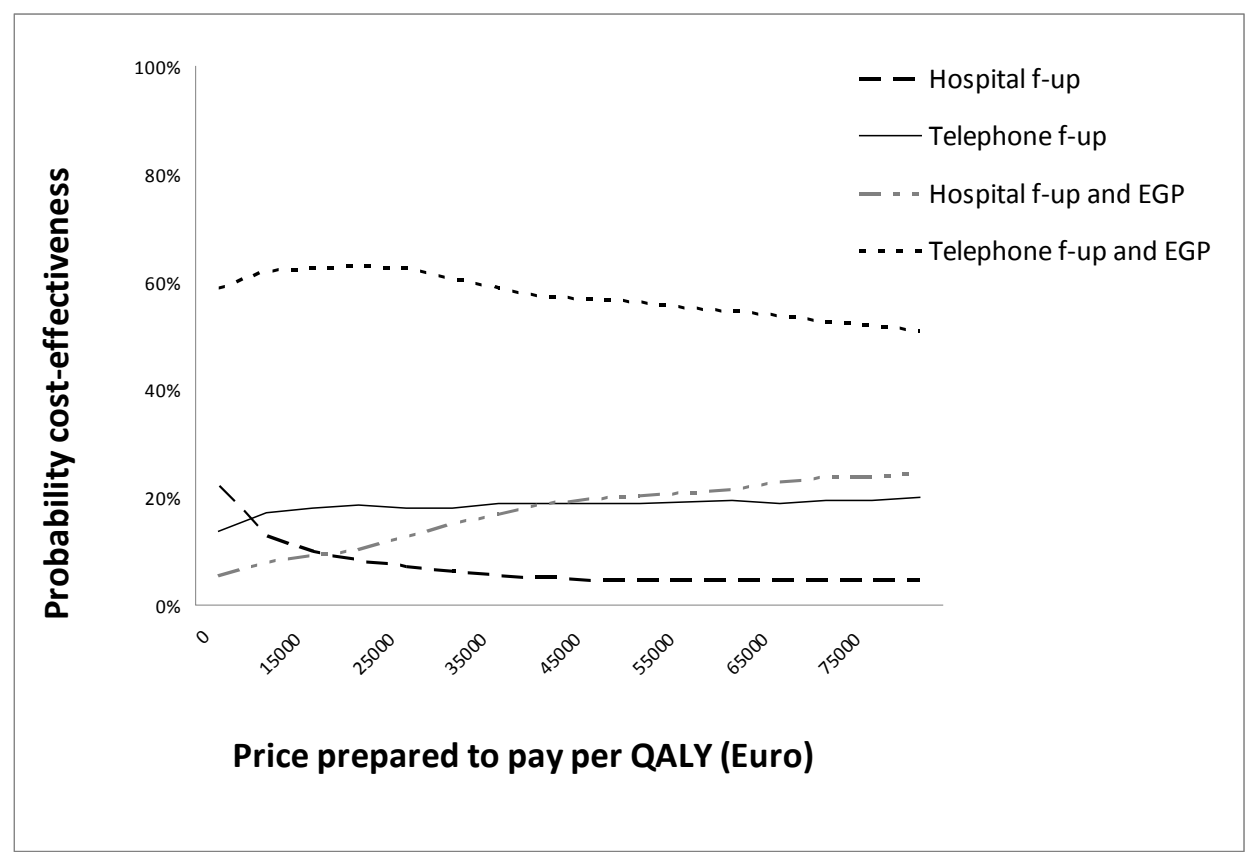

Figure 1. Cost-effectiveness acceptability curves base case analysis 


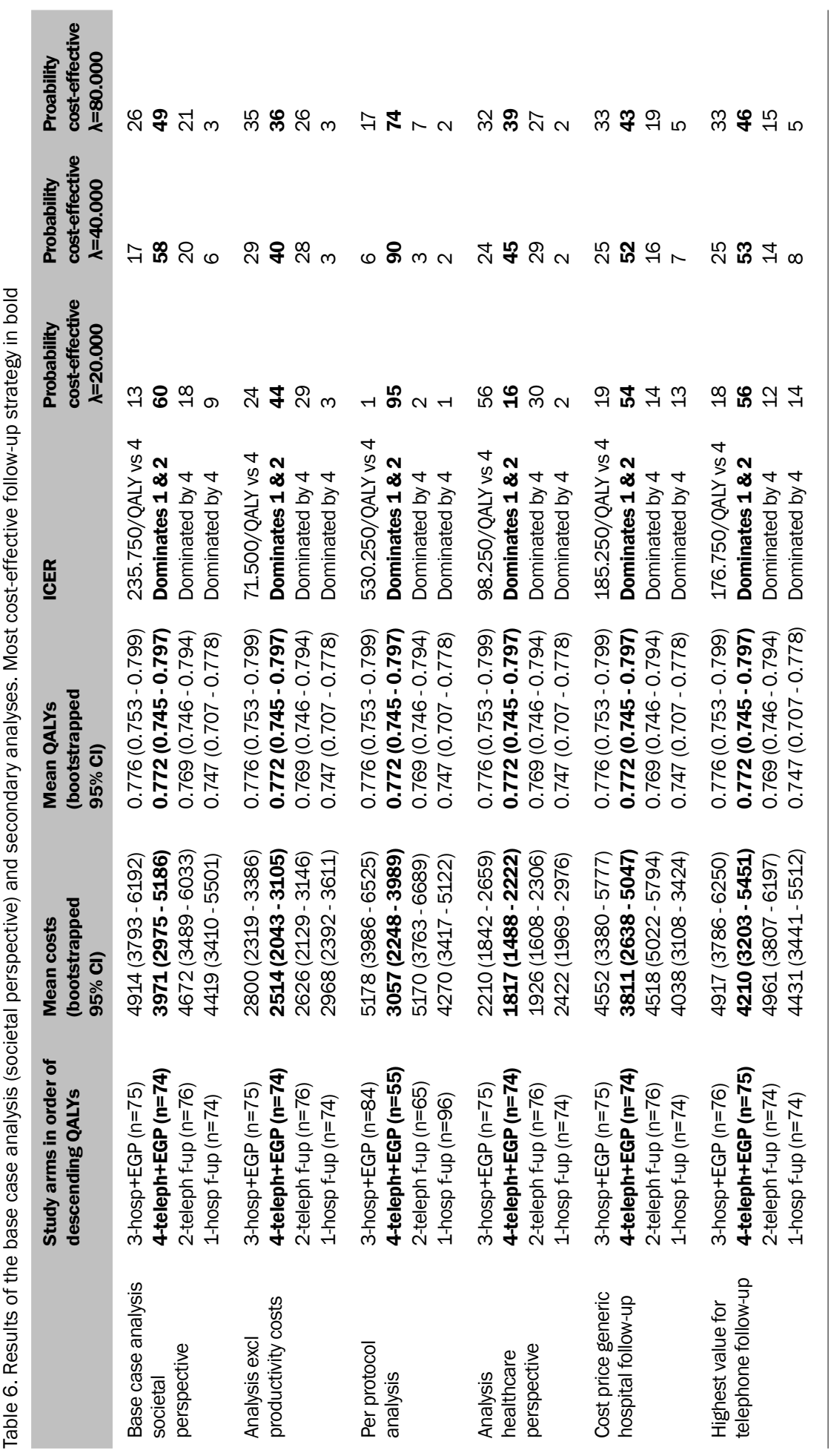




\section{Secondary analyses}

Per protocol analysis, analysis excluding productivity costs and analysis from a health care perspective all showed that telephone follow-up plus EGP was the preferred followup strategy, assuming a QALY threshold of $€ 40.000$. Furthermore, one-way sensitivity analyses showed that changing unit prices for telephone follow-up and hospital visits did not alter conclusions (table 6).

Subgroup analyses (table 7, page 72/73) showed that for patients with high levels of anxiety, hospital follow-up plus EGP is most cost-effective if society would be willing to pay at least $€ 33.269$ for one QALY gain compared to hospital follow-up. Given a threshold of $€ 40.000$, the probability of being cost-effective was $49 \%$. Age, level of education, and chemotherapy treatment did not influence cost-effectiveness results. Finally, telephone follow-up plus EGP was also the most cost-effective strategy in the analysis with a time horizon of 18 months.

\section{DISCUSSION}

\section{Key findings}

This comprehensive economic evaluation provided a detailed insight into the costeffectiveness of four follow-up strategies for breast cancer patients during their first year after treatment. Hospital follow-up plus EGP yielded most QALYs, but it was also the most costly follow-up strategy of the study. The ICER of hospital follow-up plus EGP versus the next best alternative, telephone follow-up plus EGP, amounted to $€ 235.750 / Q A L Y$. Hospital follow-up plus EGP was not considered to be cost-effective and telephone follow-up plus EGP was the preferred follow-up strategy. The probability of telephone follow-up plus EGP being most cost-effective ranged from $60 \%$ when applying a more conservative threshold value of $€ 20.000$ to $58 \%$ with a threshold of $€ 40.000$. Secondary and sensitivity analyses showed that these results were robust. However, for patients with high levels of anxiety after treatment, hospital follow-up plus EGP was the preferred strategy in terms of costeffectiveness.

The detailed cost analysis showed that health care costs were lower if some first year hospital follow-up visits were replaced by nurse-led telephone follow-up. This was mostly due to reduced costs for visits, concomitant laboratory tests and other diagnostics. Moreover, the combination of nurse-led telephone follow-up plus EGP was conducive to cost reductions. It may be speculated that a combination of frequent contacts with a BCN, together with comprehensive education about signs and symptoms of possible treatment side effects, may have led to fewer contacts with (more specialized) health care professionals.

Conceptually the cost differences between follow-up strategies were small. Mean cost differences from the societal perspective between telephone follow-up plus EGP and current clinical practice (hospital follow-up), were $€ 448$ for one year. However, by 
extrapolating these figures to the breast cancer population in the Netherlands (Kiemeney et al., 2008) this may represent an important overall cost difference overall. With 12.416 new breast cancer patients per year (Comprehensive Cancer Center, 2006), up to 5 million Euros could be saved annually, assuming successful implementation of this new strategy.

\section{Comparison with other studies}

To our knowledge, there are no earlier such publications on cost-effectiveness of followup for the first year after treatment. Although similar studies have been conducted, these either compared traditional follow-up to different alternative strategies (Grunfeld et al., 1999b; Koinberg et al., 2009), or involved a different follow-up time period (Beaver et al., 2009a). Grunfeld and colleagues showed that follow-up performed by the general practitioner did not increase use of other health care services, and reduced costs substantially (Grunfeld et al., 1999b). Koinberg and colleagues compared specialist nurse and medical specialist follow-ups and found that specialist nurse follow-up was approximately $20 \%$ less expensive (Koinberg et al., 2009).

Beaver and colleagues conducted a cost-minimization study, comparing traditional hospital follow-up with nurse-led telephone follow-up, and concluded that telephone follow-up may reduce the burden on busy hospital clinics but would not necessarily lead to cost savings. While women randomized to telephone follow-up reported less hospital consultations, the longer duration of telephone consultations, use of junior medical staff, and training costs of nurses resulted in higher routine costs for telephone follow-up (Beaver et al., 2009a). In contrast, our study used a fixed price for hospital follow-up visits, since it was often unclear which health care provider would perform the follow-up. Furthermore, training costs of nurses were not included in the cost price of telephone follow-up, as these costs diffuse among many patients outside the study domain. Importantly, changing cost prices for hospital and telephone consultations did not alter our conclusions. Hence, our studies show similar results in terms of resource use, however, the unit cost of a hospital visit resulted in contrasting conclusions (Beaver et al., 2009a).

\section{Concerns regarding the economic analysis}

Some unexpected cost differences were found between the study arms that were not obviously related to the interventions. In arm 1 (hospital follow-up) costs for in-hospital days were visibly higher compared to the other three strategies. In arm 1 three patients were admitted to the hospital for more than seven days due to breast cancer related complications, and were responsible for these high costs. It seems unlikely these admissions were related to the follow-up strategy, but it would have been inappropriate to exclude these costs in the base case analysis. A secondary analysis excluding these patients showed this did not alter conclusions.

From the societal perspective, indirect costs due to absence from paid work or not able to perform domestic tasks accounted for a major part of the total costs of all study arms (almost 40\%); however, these estimates showed large 95\% confidence intervals. 
The present large differences in indirect costs between groups were unexpected and might be explained by relatively small sample sizes. Excluding the productivity costs in the base case analysis would have violated the pre-defined protocol and the Dutch guidelines for economic evaluation (Oostenbrink et al., 2004). Moreover, the mean age of the sample was 56 years old and one in three women was in paid employment at the time of her breast cancer diagnosis. To address the uncertainty induced by these high costs, a secondary analysis excluding these specific costs was also reported. This analysis showed that the ICER for hospital follow-up plus EGP versus telephone follow-up plus EGP dropped to 71.500/QALY. Since this ICER is still above the Dutch QALY threshold telephone followup plus EGP remained the preferred strategy.

One might argue that, since no significant differences were found in the clinical analysis and QALY differences were small, a cost-minimization analysis would have been more appropriate. However, the clinical effectiveness study used a 2x2 factorial design and therefore did not compare the four strategies separately as was done in this economic evaluation. Hence, the study was not designed to show equivalence in effectiveness of the four strategies. Therefore, a cost-minimization analysis would have been inappropriate (Briggs \& O'Brien, 2001). Finally, one might be concerned that the use of the EQ-5D and the resulting estimates of QALYs are not sensitive enough in these patients to identify differences in their HRQoL. However, the EQ-5D was found to be a responsive measure in this breast cancer population, able to detect improvements and deteriorations in health (Kimman et al., 2009). The EQ-5D is also the recommended outcome measure for economic evaluations (Earnshaw \& Lewis, 2008) and commonly used in cancer populations (Deconinck et al., 2010; Freedman et al., 2010; TOMBOLA, 2009). The use of the EQ-5D in this study therefore allows comparison to other studies.

\section{Strengths}

It is generally recognized that most breast cancer follow-up related costs are incurred in the first two years after treatment (Lidgren et al., 2007). Also, most improvement in psychosocial status is reported for the first year after treatment (Ganz et al., 1996). Hence, the present study focused on a key time period after treatment for medical decision making. This economic evaluation was performed according to published international guidelines for trial-based economic evaluations (Earnshaw \& Lewis, 2008), within the framework of a multicenter randomized controlled trial. The randomized setting minimized selection bias and due to the pragmatic nature of the trial it closely reflects the effectiveness and resource use that would be observed in ordinary daily practice. We compared four alternative strategies, which is relatively uncommon, in order to address the often proposed disadvantage of trial-based economic evaluations that they represent only a limited form of analysis by comparing few alternatives (Sculpher et al., 2006). Furthermore, by using micro costing to value resources and presenting tables with resource use and cost prices, we have provided detailed information on follow-up costs and have been as transparent as possible. Case-by-case calculations for the purpose of generalizability are possible. 


\section{CONCLUSIONS}

Results of this study may bridge an important evidence gap on cost-effectiveness of alternative follow-up strategies after breast cancer treatment. Our results and those of others (Beaver et al., 2009a; Grunfeld et al., 1999b; Koinberg et al., 2009) increasingly underscore the importance of critically assessing current guidelines for breast cancer follow-up. This economic analysis showed that nurse-led telephone follow-up plus an EGP instead of traditional hospital clinic visits may lead to substantial cost reductions in the Netherlands. 


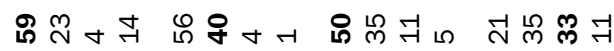

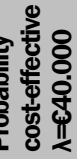

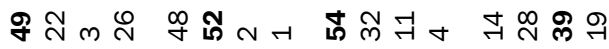

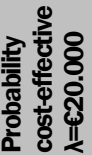

ลำ 욤 \&

哸

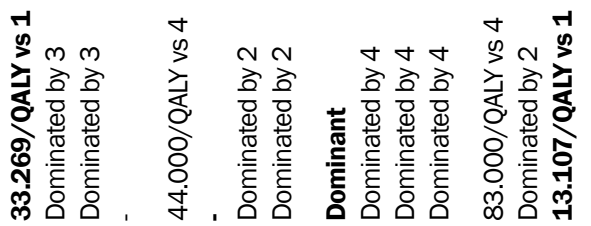

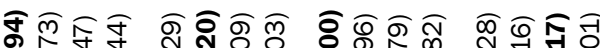

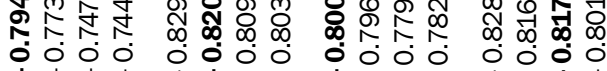

ง r5s

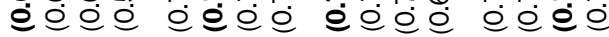

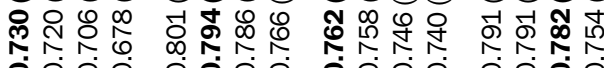

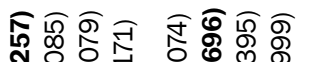

쇼용

$\exists$ 守

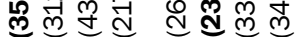

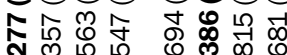

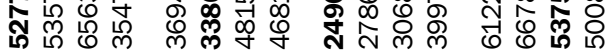
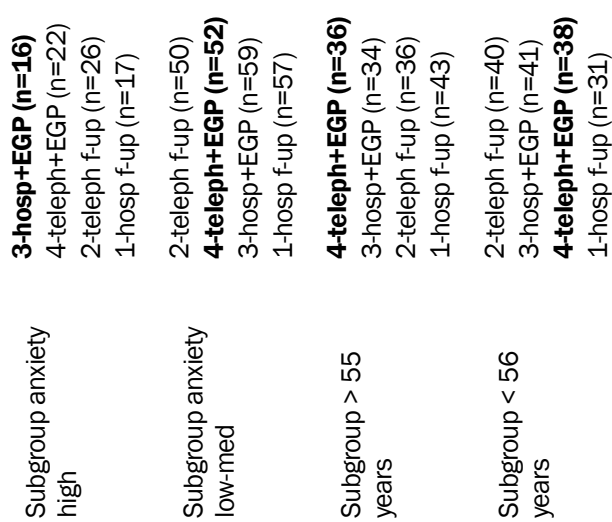

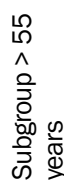

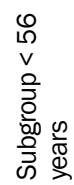




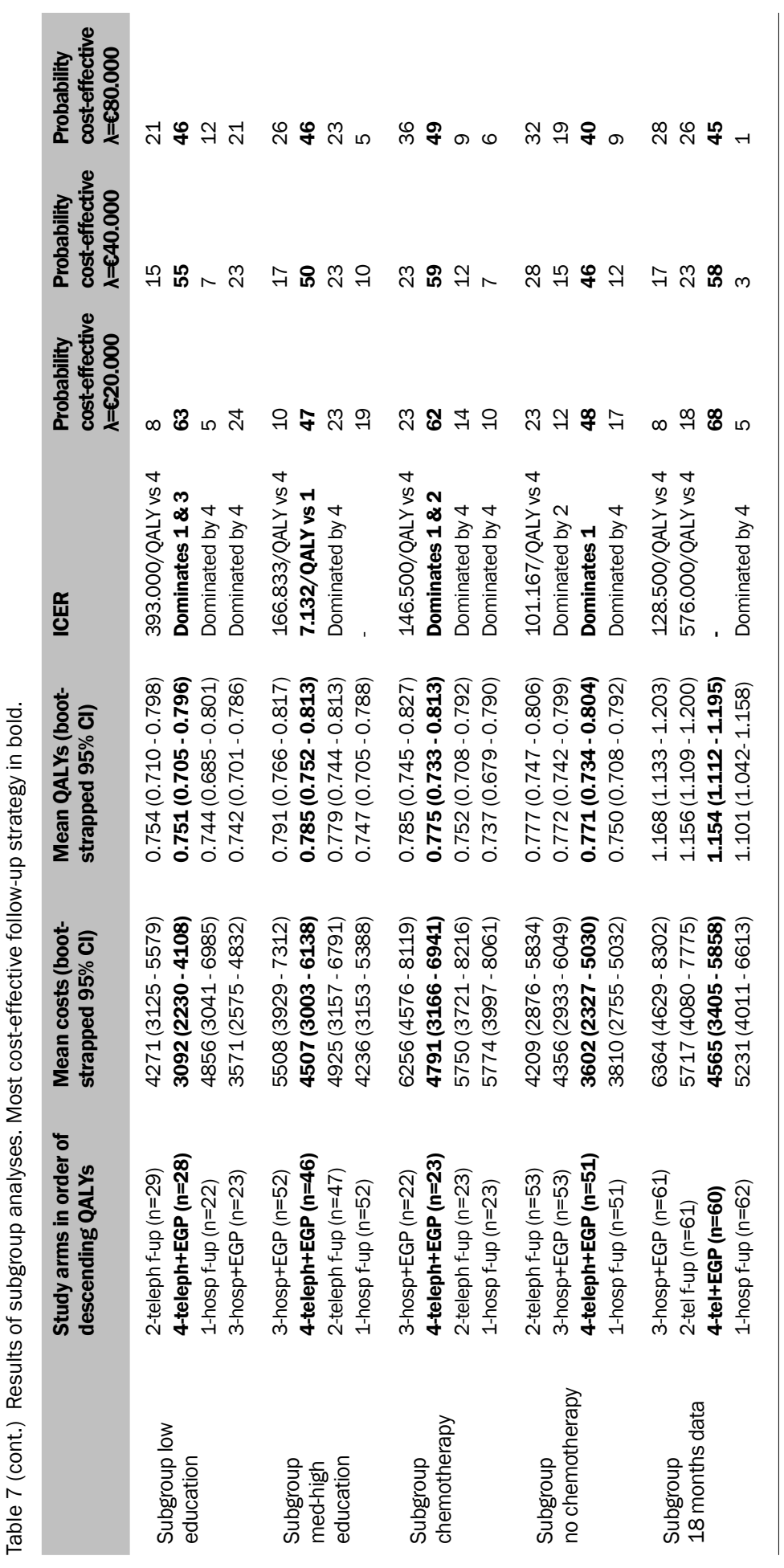




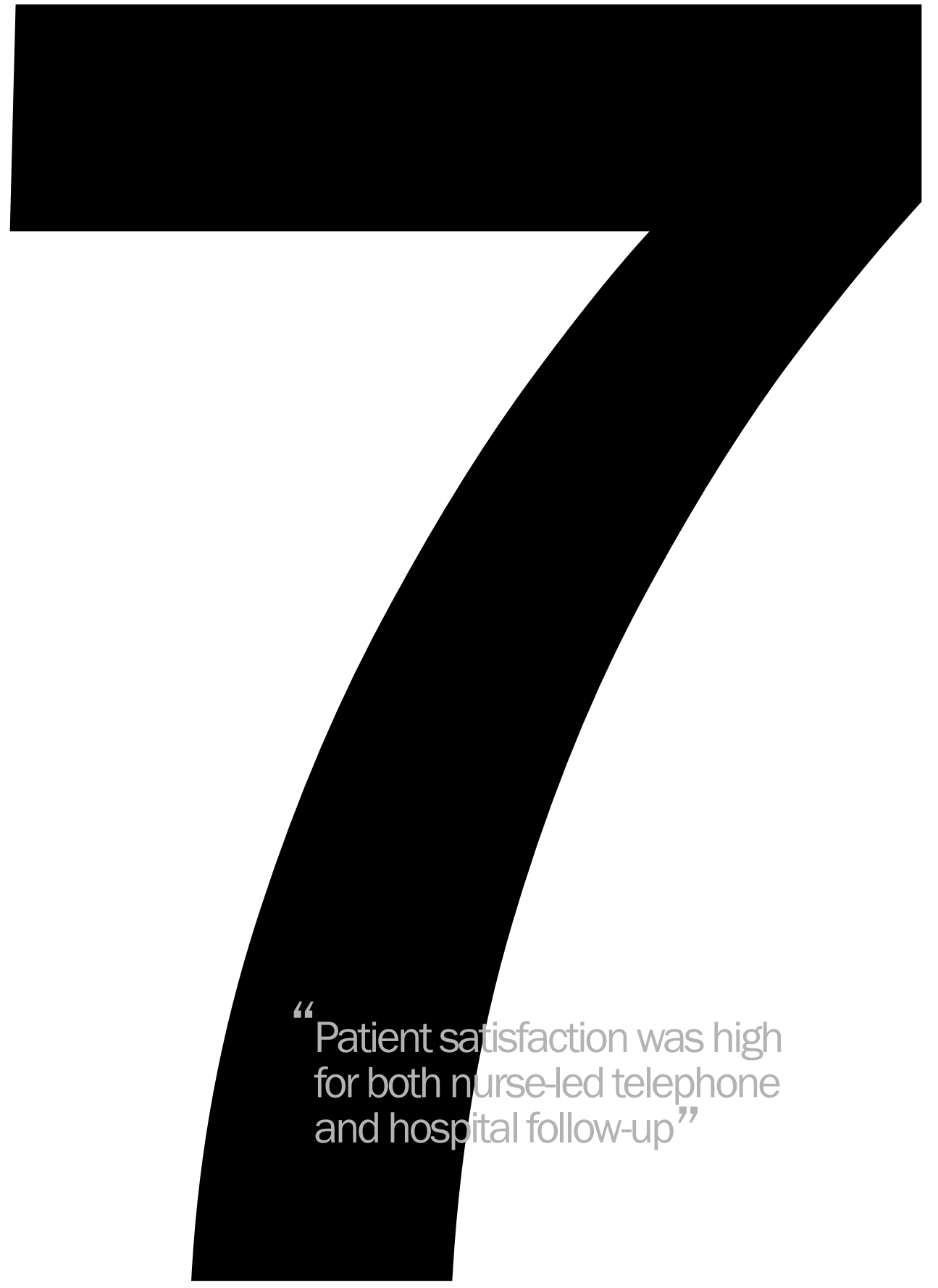




\title{
Patient satisfaction with nurse-led telephone follow-up after curative treatment for breast cancer
}

KIMMAN ML, BLOEBAUM MMF, DIRKSEN CD, HOUBEN RMA, LAMBIN Ph, BOERSMA L.

BMC CANCER 10(1): 174

\begin{abstract}
[Background] Current frequent follow-up after treatment for breast cancer does not meet its intended aims, but does depend on expensive and scarce specialized knowledge for routine history taking and physical examinations. The study described in this paper compared patient satisfaction with a reduced follow-up strategy, i.e. nurse-led telephone follow-up, to satisfaction with traditional hospital follow-up. [Methods] Patient satisfaction was assessed among patients ( $n=299$ ) who were participants of a randomized controlled trial investigating the cost-effectiveness of several follow-up strategies in the first year after treatment for breast cancer. Data on patient satisfaction were collected at baseline, 3,6 and 12 months after treatment, using the Dutch version of Ware's Patient Satisfaction Questionnaire III (PSQ III). In addition to general satisfaction, the PSQ III reports on satisfaction scores for technical competence, interpersonal aspects, and access of care. Regression analysis was used to predict satisfaction scores from whether or not nurse-led telephone follow-up was received. [Results] Nurse-led telephone follow-up had no statistically significant influence on general patient satisfaction $(p=0.379)$, satisfaction with technical competence $(p=0.249)$, and satisfaction with interpersonal aspects $(p=0.662)$. Regarding access of care, patient satisfaction scores were significantly higher for patients receiving telephone follow-up $(p=0.015)$. However, a mean difference at 12 months of 3.1 points was judged to be not clinically relevant. [Conclusions] No meaningful differences were found in satisfaction scores between nurse-led telephone and hospital follow-up in the first year after breast cancer treatment. With high satisfaction scores and the potential to substantially reduce clinic visits, nurse-led telephone follow-up may be an acceptable alternative to traditional hospital follow-up.
\end{abstract}




\section{INTRODUCTION}

In most countries, follow-up after curative treatment for breast cancer consists of frequently scheduled follow-up visits (2-4 times a year) and an annual routine mammography (Pestalozzi \& Castiglione, 2008). The main objectives of these visits are the early detection of a locoregional recurrence or a second primary breast cancer, detection and registration of side effects of treatment, and provision of information and psychological support (Pestalozzi \& Castiglione, 2008; Struikmans et al., 2008). However, there is much debate whether these objectives are met in current clinical practice (Brada, 1995; Dewar, 1995). First of all, routine follow-up with clinical examination has been shown not to contribute to improved survival (GIVIO, 1994; Montgomery et al., 2009; Rosselli Del Turco et al., 1994; te Boekhorst et al., 2001; Wheeler et al., 1999). Additionally, outpatient clinic visits have been identified as a transition time of high stress, generating increased anxiety because of the risk of detecting a recurrence (Allen, 2002). Also, some studies have suggested that outpatient clinic visits are insufficient to provide psychological support after breast cancer treatment, since these visits are often brief and do not include assessment of psychosocial problems (Pennery \& Mallet, 2000). Hence, current frequent follow-up visits seem to miss their most important goals, but do depend heavily on expensive and scarce specialized knowledge for routine history taking and physical examinations. As a result, the improvement in quality and efficiency of breast cancer follow-up care has been a government priority setting in many countries (Houlihan, 2009; Gezondheidsraad, 2007). Alternative strategies for follow-up such as nurse-led and telephone follow-up have been proposed (Beaver et al., 2009b; Koinberg et al., 2004; Montgomery et al., 2008a). To improve healthcare, feedback from patients on these alternative models of follow-up is essential. Feedback evaluates the quality of the care provided, and can isolate problem areas and help generating ideas for further improvement (Crow et al., 2002; Sitzia \& Wood, 1997). Moreover, patients may respond better to treatment and comply better with instructions when they are satisfied with their care and treatment setting, improving their health outcomes (Jackson et al., 2001; Kizer, 2002; Worthington, 2004).

Patient satisfaction with nurse-led follow-up and telephone services was found to be high (Cox \& Wilson, 2003; Lewis et al., 2009), and good outcomes have been reported in terms of detecting medical problems and providing support to cancer patients (James et al., 1994; Marcus et al., 2009). Recent studies in breast cancer patients have also shown that telephone follow-up by specialist breast care nurses (BCNs) was well-received by patients. A randomized clinical trial (RCT) by Beaver et al. (2009b), comparing nurse-led telephone follow-up with hospital follow-up showed that telephone follow-up significantly improved satisfaction, and it produced no excess anxiety compared with hospital followup. A prospective cohort study by Montgomery et al. (2008a) showed that an automated telephone system was easy to use and liked by most (71\%) patients.

These studies provided positive results regarding patient satisfaction for follow-up after breast cancer for women on average one to two years after diagnosis. However, there is 
evidence that most physical and psychological recovery is achieved within the first year following treatment (Ganz et al., 1996). Therefore, the introduction of nurse-led telephone follow-up in the first year after treatment may affect patient satisfaction and quality of care differently than when applied in a later stage. It is expected that telephone follow-up by a $\mathrm{BCN}$, who is familiar with the patient, can be appropriate to address psychological consequences after treatment (Beaver et al., 2009b). Moreover, especially in the first year, telephone follow-up has the potential to reduce hospital visits.

This paper focused on patient satisfaction with nurse-led telephone follow-up compared to hospital follow-up in the first year after breast cancer treatment. Patient satisfaction was measured using a shortened (Dutch) version of the validated Patient Satisfaction Questionnaire (PSQ III) constructed by Ware et al. (Hagedoorn et al., 2003). The PSQ III measures the multidimensional concept of patient satisfaction, capturing the most important characteristics of services and providers that might influence patient satisfaction with care. It is believed to reflect quality of care and patients' preferences (Ware et al., 1983).

\section{METHODS}

\section{Recruitment, design and sample}

Patient satisfaction was assessed using data from patients who were participants of a randomized controlled trial (RCT) investigating, among other things, the cost-effectiveness of nurse-led telephone follow-up after breast cancer (ISRCTN 74071417). A predefined (secondary) aim of the trial was to compare patient satisfaction between nurse-led telephone and hospital follow-up. Details of the trial design and protocol execution have been reported previously (Kimman et al., 2007b). The study was a multicenter randomized trial, with a 2x2 factorial design. Between 2005 and 2008, 320 women were recruited through seven hospitals and two radiotherapy clinics in the South of the Netherlands. Participants were eligible for inclusion if they had completed breast cancer treatment with curative intent less than six weeks prior to randomization with a WHO performance score between 0-2, and were fluent in speaking and reading Dutch. Exclusion criteria were distant metastases, and/or participation in another clinical trial or medical illness requiring more frequent follow-up. All eligible patients received detailed study information, including an information sheet on follow-up after breast cancer treatment. This information sheet described the purpose and effectiveness of breast cancer follow-up and signs and symptoms of possible recurrences.

After written informed consent was obtained, participants were randomly assigned to one of four follow-up strategies (study arms) during the first 12 months after treatment; i.e. 1) hospital follow-up every three months, including mammography at 12 months; 2) nurseled telephone follow-up every three months, plus hospital visit and mammography at 12 months; 3) arm 1 plus educational group program (EGP); 4) arm 2 plus EGP.

Randomization by minimization (Taves, 1974) was performed by the Comprehensive 
Cancer Center Limburg using a computerized randomization program (ALEA). Patients were pre-stratified by hospital and treatment modality (surgery, surgery + radiotherapy, surgery + chemotherapy, and surgery + radiotherapy + chemotherapy). The study protocol was approved by the Medical Ethical Review Board of MAASTRO Clinic (NL). All participating centers signed a local feasibility declaration, according to Dutch law and regulations, prior to inclusion of the first patient.

For the purpose of this analysis, hospital follow-up (arms 1 and 3) was compared to telephone follow-up (arms 2 and 4). In total, 162 patients were randomized to nurse-led telephone follow-up and 158 patients to traditional hospital follow-up.

\section{Procedures and intervention}

In the Netherlands, follow-up after breast cancer in the first year after treatment consists of routine follow-up visits to the hospital (i.e. at 3, 6, 9 and 12 months after treatment) (Struikmans et al., 2008). A mammography is made at 12 months after the start of treatment, which is combined with a hospital follow-up visit. The provider of follow-up alternates between the surgeon, BCN, medical oncologist and radiation oncologist.

In patients randomized for hospital follow-up, follow-up was performed according to the above described Dutch guidelines. The follow-up visits consisted of physical examination and medical history and had a scheduled duration of approximately 10 minutes. In patients randomized for telephone follow-up, follow-up at 3, 6 and 9 months was performed by telephone, by a BCN or nurse practitioner (NP) working at this hospital, preferably the same nurse at each follow-up moment. At 12 months, a mammography was made and combined with a hospital follow-up. The telephone follow-up included a semi-structured interview in which physical -especially loco-regional- and psychosocial symptoms, treatment side effects and compliance with hormonal therapy were discussed. Furthermore, the BCN informed about general well-being of the patient, her family life, relationships, and work reintegration. Time scheduled for the telephone interview was approximately 15-20 minutes. If the patient had specific complaints or did not feel reassured, an additional appointment was made for her to come to the hospital. In order to adequately perform the telephone interview, all participating nurses attained four halfday training sessions, specifically developed for this study. In this training BCNs were informed on the most recent developments in breast cancer treatment and follow-up, and practiced their telephone communication skills with a simulation patient. Twenty-one BCNs from seven hospitals were trained.

\section{Outcome measures of effect}

\section{Patient satisfaction}

To assess patient satisfaction the Dutch version of the validated Ware's Patient's Satisfaction Questionnaire III (PSQ III) was used. The PSQ III captures the most important characteristics of services and providers that might influence patient satisfaction with care. The Dutch version has left out financial aspects of the original questionnaire (i.e. 
questions 4, 10, 14, 19, 24, 27, 32 and 44), since in the Dutch health care system the personal financial situation is not directly related to the provision and quality of medical care. The structure and reliability of the PSQ III has been tested in a large sample of cancer patients in the Netherlands who were on average 8.6 months after treatment. Of this sample $31.1 \%$ were breast cancer patients. The PSQ III appeared an appropriate measure of cancer patient's satisfaction (Hagedoorn et al., 2003).

The PSQ III is a three factor model: besides general satisfaction (PSQ total), it consists of satisfaction with interpersonal aspects (IA) of the health care professional (i.e. providing explanations, listening skills, hasty behavior, empathy and respect), technical competence (TC) (i.e. knowledge of latest treatment techniques, competences of specialist/nurse) and satisfaction with access of care (AC) (i.e. easy and quick access to care, quality of care, waiting time). The questionnaire contains 43 favorably and unfavorably worded statements. Respondents are asked to indicate their agreement with the statements with respect to the care they received. The statements in the questionnaire all assume that medical care is provided by a doctor. To adjust to the study environment, the 'nurse' was added to the questionnaire. For example, 'the doctor/nurse who treats me has a genuine interest in me as a person' (question 17). Items are included in the questionnaire in random order and the answer alternatives range from 1 (strongly agree) to 5 (strongly disagree) (Hagedoorn et al., 2003). Answers to favorably worded statements are reversecoded, so that a higher number indicates more satisfaction. Sum scores were calculated for the PSQ total scale and for the three dimensions and subsequently transformed into a 100-point scale.

Possible response bias was investigated by using the matched-pairs method (Ware, 1978). This methods checks whether a respondent tends to agree (or disagree) with two statements known to define opposite ends of the same satisfaction continuum, e.g. 'Doctors/nurses carefully listen to what I have to say' and 'Doctors/nurses sometimes ignore what I tell them'. The PSQ III includes five matched pairs. Each of these pairs is assigned a score 0 if no response bias is present, and 1 if response bias is present. Consequently, the theoretical range of response bias is 0 to 5 ; a score of 0 indicates no response bias, whereas a score of 2 or higher represents substantial bias.

The PSQ III was filled out before randomization at baseline, and 3, 6 and 12 months after treatment. Patients received the questionnaire at home approximately one week after the follow-up visit or telephone interview and were asked to return it by mail in an enclosed envelope.

\section{Protocol compliance}

Data on the actual follow-up received, thus number of hospital visits and telephone contacts with a BCN, were collected from patient files. 


\section{Statistical analysis}

The sample size for this study was determined by the sample size of the RCT, which was based on its primary outcome measure, i.e. health-related quality of life (HRQoL) at 12 months after end of treatment (Kimman et al., 2007b). This paper deals with patient satisfaction at 12 months after treatment. We hypothesized that satisfaction with nurseled telephone follow-up, regarding general satisfaction, interpersonal aspects, technical competences, and access of care, could be different from satisfaction with hospital followup. A difference in satisfaction scores of at least 0.5 standard deviation (SD), a medium effect, was considered to be clinically relevant. Assuming an SD of 17.9 for both groups (Hagedoorn et al., 2003), post hoc calculations showed that a sample of 299 patients from the RCT allowed to demonstrate a clinically relevant difference between nurse-led telephone and hospital follow-up (i.e. 8.95 points difference) with 90\% statistical power and an $\alpha$ of 0.05 .

Data were entered in a database by a professional center for data and information management and analyzed using SPSS version 17.0. Missing values within the survey's subscales were replaced using the regression function in SPSS if no more than half of the items were missing. If more than half of the items were missing the subscale was considered missing. If three or four PSQ III questionnaires were not returned, the patient was judged to be lost to follow-up. Missing data (i.e. subscales) from one or two questionnaires or missing covariates were imputed by means of Rubin's multiple imputation procedure (Schafer, 1999).

Differences in patient characteristics between telephone and hospital follow-up were compared using the $\mathrm{X}^{2}$ test and independent sample t-test. Regression analyses were used to predict outcome differences by including or excluding the intervention. Linear mixed models were fitted with telephone follow-up (yes/no) as a fixed factor, and patient as random factor. In addition, time since end of treatment, age, hospital, treatment modality, education level, and the outcome variable at baseline were brought into the model. In the primary analysis data were analyzed according to the intention to treat principle. However, since protocol violation may bias the results (in either direction), per protocol analyses including only patients who properly followed the study protocol were also performed and reported (Jones et al., 1996; Piaggio et al., 2006).

\section{RESULTS}

\section{Patients}

Data from 299 patients were available for the purpose of this analysis. In the RCT, 21 of 320 randomized patients had dropped out of the trial due to various reasons (e.g. development of metastases, recurrence, or three or more missing questionnaires). Data of 149 patients randomized to hospital follow-up and data of 150 patients randomized to telephone follow-up were available for the evaluation of patient satisfaction. Mean sample 
age was 56 years. Sociodemographic and treatment characteristics as well as baseline satisfaction scores were similar in the two groups (table 1).

Table 1. Sociodemographic and treatment characteristics of participants $(n=299)$ according to hospital or telephone follow-up. Values are numbers (percentages) unless stated otherwise.

\begin{tabular}{|c|c|c|c|c|}
\hline & $\begin{array}{l}\text { Total group } \\
(\mathrm{n}=299)\end{array}$ & $\begin{array}{l}\text { Hospital } \\
\text { follow-up } \\
(n=149)\end{array}$ & $\begin{array}{l}\text { Telephone } \\
\text { follow-up } \\
(n=150)\end{array}$ & p-value \\
\hline \multicolumn{5}{|l|}{ Age at randomization (years) } \\
\hline Mean (SD) & $56(9.9)$ & $56(10.7)$ & $55(9.0)$ & 0.50 \\
\hline Range & $23-78$ & $23-78$ & $34-75$ & 0.06 \\
\hline$<45$ & $36(12)$ & $18(12)$ & $18(12)$ & \\
\hline $45-64$ & $203(68)$ & $93(62)$ & $110(73)$ & \\
\hline$\geq 65$ & $60(20)$ & $38(26)$ & $22(15)$ & \\
\hline Level of education & & & & 0.36 \\
\hline Low & $102(34)$ & $45(30)$ & $57(39)$ & \\
\hline Middle & $118(40)$ & $62(42)$ & $56(37)$ & \\
\hline High & $79(26)$ & $42(28)$ & $37(24)$ & \\
\hline Marital status & & & & 0.15 \\
\hline Married & $212(71)$ & $109(73)$ & $103(69)$ & \\
\hline Unmarried & $33(11)$ & $16(11)$ & $17(11)$ & \\
\hline Cohabiting & $29(10)$ & $9(6)$ & $20(13)$ & \\
\hline Widowed & $25(8)$ & $15(10)$ & $10(7)$ & \\
\hline Treatment modality & & & & 0.99 \\
\hline Surgery & $29(10)$ & $15(10)$ & $14(9)$ & \\
\hline Surgery + radiotherapy (RT) & $178(60)$ & $89(60)$ & $89(60)$ & \\
\hline Surgery + chemotherapy $(\mathrm{CH})$ & $15(5)$ & $7(5)$ & $8(5)$ & \\
\hline Surgery + RT + CH & $77(25)$ & $38(25)$ & $39(26)$ & \\
\hline Hormonal therapy & & & & 0.60 \\
\hline Yes & $94(31)$ & $50(34)$ & $44(29)$ & \\
\hline No & $205(69)$ & $99(66)$ & $106(71)$ & \\
\hline \multicolumn{5}{|l|}{$\begin{array}{l}\text { Baseline satisfaction scores } \\
\text { (mean (SD)) }\end{array}$} \\
\hline General satisfaction & $77.2(19.5)$ & $77.7(14.2)$ & $76.7(18.9)$ & 0.65 \\
\hline Interpersonal aspects & $81.8(17.5)$ & $81.4(17.6)$ & $82.2(17.5)$ & 0.70 \\
\hline Access of care & $76.9(14.0)$ & $77.4(14.2)$ & $76.4(13.8)$ & 0.54 \\
\hline Technical competence & 77.0 (16.3) & $76.7(17.2)$ & 77.4 (15.4) & 0.69 \\
\hline
\end{tabular}

\section{Compliance to the protocol}

Ten patients randomized for telephone follow-up preferred to receive hospital followup instead, and case record forms indicated that 20 patients with telephone follow-up received only one telephone follow-up contact, which was considered as protocol violation. Hence, 120 of the 150 patients in the telephone follow-up group, received telephone follow-up according to the protocol. Since hospital follow-up represented usual care, no 
protocol violations were apparent in this group.

The 30 patients who violated the protocol in the telephone group did not differ from other patients in this group regarding age, education, treatment modality and satisfaction scores at baseline (all p-values $>0.05$ ).

Table 2 shows the mean number of total hospital visits and telephone contacts with the breast care nurse per group for the study period of one year. In the hospital group patients had on average 5.9 visits to the hospital, of which four visits were conform protocol and 1.9 were additional visits. In the telephone group patients had on average 2.4 telephone contacts with the BCN and 3.4 visits to the hospital, of which one hospital visit was conform protocol and 2.4 were additional visits.

Table 2. Number of contacts with medical specialist (MS) and breast care nurse (BCN) according to followup group (hospital and telephone) in one year. Numbers are means and standard deviations.

\begin{tabular}{|c|c|c|c|c|c|c|}
\hline & \multicolumn{3}{|c|}{ Hospital follow-up } & \multicolumn{3}{|c|}{ Telephone follow-up } \\
\hline & $\begin{array}{l}\text { Conform } \\
\text { protocol }\end{array}$ & $\begin{array}{l}\text { Additional } \\
\text { contacts }\end{array}$ & $\begin{array}{c}\text { Total } \\
\text { contacts }\end{array}$ & $\begin{array}{l}\text { Conform } \\
\text { protocol }\end{array}$ & $\begin{array}{c}\text { Additional } \\
\text { contacts }\end{array}$ & $\begin{array}{c}\text { Total } \\
\text { contacts }\end{array}$ \\
\hline \multicolumn{7}{|l|}{ Intention to treat analysis } \\
\hline Visits hospital (MS or BCN) & 4 & 1.9 & $5.9(2.2)$ & 1 & 2.4 & $3.4(2.4)$ \\
\hline Telephone contact BCN & 0 & 0.1 & $0.1(0.4)$ & 3 & $-0.6^{a}$ & $2.4(1.1)$ \\
\hline \multicolumn{7}{|l|}{ Per protocol analysis } \\
\hline Visits hospital (MS or BCN) & 4 & 1.9 & $5.9(2.2)$ & 1 & 2.0 & $3.0(2.3)$ \\
\hline Telephone contact BCN & 0 & 0.1 & $0.1(0.4)$ & 3 & $-0.2^{a}$ & $2.8(0.7)$ \\
\hline
\end{tabular}

a Negative numbers imply fewer contacts than set by the protocol

\section{Patient satisfaction}

Since patients who violated the protocol for telephone follow-up did not differ from patients who adhered to telephone follow-up, results from the intention to treat analyses are discussed as the primary outcomes in this section. Nevertheless, table 3 shows results of both intention to treat and per protocol analyses.

In the first year after treatment patient satisfaction scores were high in both groups in all subscales of the PSQ III, at all time points (see figure 1). Table 3 (page 84) shows satisfaction scores at 12 months after treatment. General patient satisfaction at 12 months was $75.3(\mathrm{SD}=19.6)$ in the hospital follow-up group and $76.4(\mathrm{SD}=19.7)$ in the telephone follow-up group. Patient satisfaction regarding technical competence was 73.7 $(S D=17.9)$ for the hospital follow-up group and $75.8(S D=16.8)$ for the telephone followup group. Satisfaction with interpersonal aspects was $78.7(\mathrm{SD}=18.5)$ for the hospital follow-up group and 78.9 ( $S D=17.6)$ for the telephone group. Regarding access of care satisfaction for the hospital follow-up group was $73.3(\mathrm{SD}=15.7)$ and for the telephone follow-up group 76.4 (SD=15.6). Regression analysis showed that nurse-led telephone 
follow-up had no statistically significant influence on general patient satisfaction ( $p=0.379)$, satisfaction with technical competence $(p=0.249)$, and satisfaction with interpersonal aspects $(p=0.662)$. Regarding access of care, patient satisfaction was significantly higher for patients receiving telephone follow-up $(p=0.015)$. However, a mean difference at 12 months of 3.1 points may be statistically significant, but was not considered clinically relevant (i.e. < medium effect).

In the per protocol analyses, ten patients who had refused telephone follow-up after randomization were analyzed in the hospital follow-up group, while the 20 patients who had not properly received telephone follow-up were excluded from the analyses. Per protocol analyses showed almost identical results to intention to treat analyses. However, in contrast to the intention to treat analysis, higher patient satisfaction scores in the telephone group regarding access of care were not significant $(p=0.060)$.

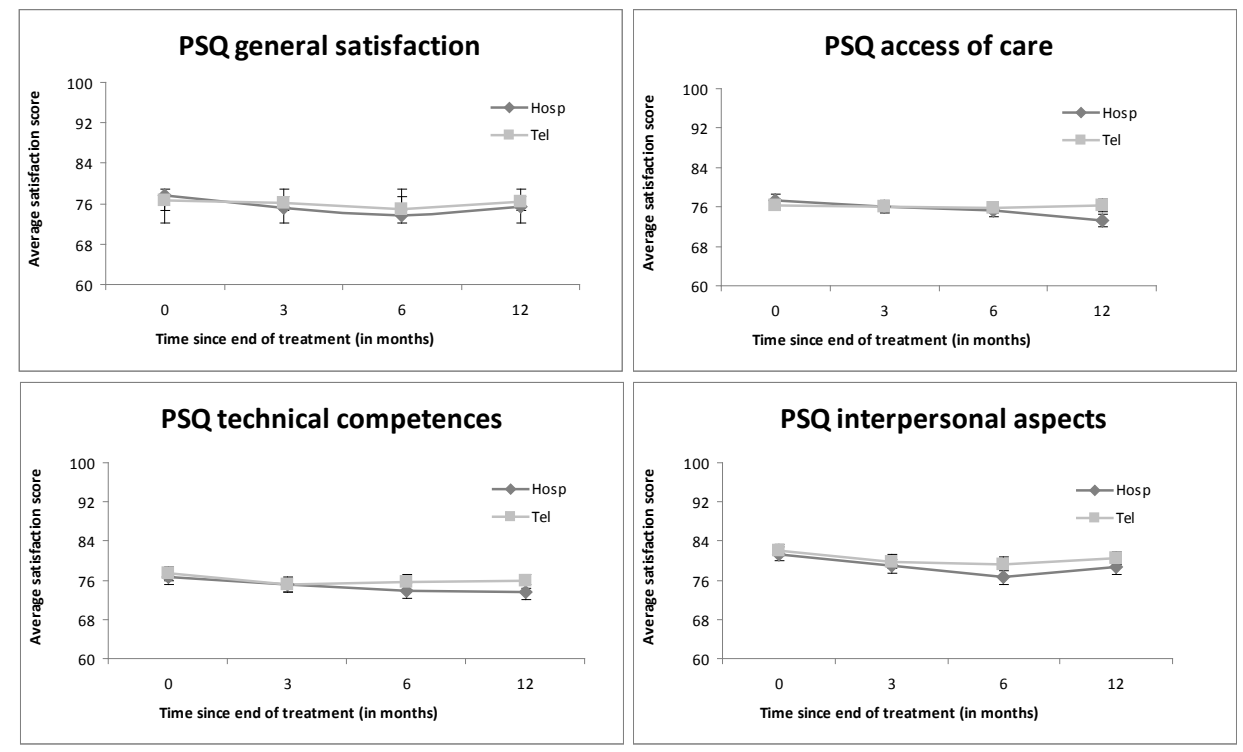

Figure 1. Average satisfaction scores with general satisfaction, access of care, technical competences, and interpersonal aspects, at baseline, 3,6 and 12 months after treatment, for hospital follow-up (black line) compared to nurse-led telephone follow-up (grey line). Error bars represent 95\% confidence intervals.

\section{Response bias}

Response bias in the questionnaire was tested on the 12 months data. We found neglectable response bias scores for the tendency to disagree regardless of content ( $0.7 \%$ of respondents), but $14 \%$ of respondents showed substantial response bias, i.e. agreed with two or more (out of five) opposite statements regardless of their content. Nevertheless, an analysis with a calibrated sample of respondents without response bias $(n=257)$ showed identical results to the main analysis regarding all factors of satisfaction (data not shown). 
Table 3. Outcome findings at 12 months after treatment, by study group adjusted for treatment, hospital, outcome variable at baseline, age, educational level, support from partner and time since treatment.

\begin{tabular}{|c|c|c|c|c|c|}
\hline & $\begin{array}{l}\text { Mean (SD) } \\
\text { telephone } \\
\text { f-up (12 } \\
\text { months) }\end{array}$ & $\begin{array}{l}\text { Mean (SD) } \\
\text { hospital } \\
\text { f-up (12 } \\
\text { months) }\end{array}$ & $\begin{array}{l}\text { Parameter } \\
\text { estimate }\end{array}$ & $\begin{array}{l}95 \% \\
\text { Confidence } \\
\text { Interval for } \\
\text { difference }^{a}\end{array}$ & p-value \\
\hline Intention to treat analysis & $(n=150)$ & $(n=149)$ & & & \\
\hline \multicolumn{6}{|l|}{ General satisfaction } \\
\hline $\begin{array}{l}\text { Telephone vs hospital f-up } \\
\text { Interpersonal aspects }\end{array}$ & $76.4(19.7)$ & $75.3(19.6)$ & 1.86 & -2.30 to 6.03 & 0.379 \\
\hline $\begin{array}{l}\text { Telephone vs hospital f-up } \\
\text { Access of care }\end{array}$ & $80.5(17.6)$ & 78.7 (18.5) & 0.91 & -3.18 to 5.00 & 0.662 \\
\hline $\begin{array}{l}\text { Telephone vs hospital f-up } \\
\text { Technical competence }\end{array}$ & $76.4(15.6)$ & $73.3(15.7)$ & 3.71 & .71 to 6.70 & 0.015 \\
\hline Telephone vs hospital f-up & $75.8(16.8)$ & 73.7 (17.9) & 2.13 & -1.51 to 5.77 & 0.249 \\
\hline Per protocol analysis & $(n=120)$ & $(n=159)$ & & & \\
\hline \multicolumn{6}{|l|}{ General satisfaction } \\
\hline $\begin{array}{l}\text { Telephone vs hospital f-up } \\
\text { Interpersonal aspects }\end{array}$ & $76.2(19.7)$ & $75.7(19.9)$ & 1.13 & -3.14 to 5.39 & 0.604 \\
\hline Telephone vs hospital f-up & $81.3(17.5)$ & $79.2(18.2)$ & 0.55 & -3.63 to 4.73 & 0.796 \\
\hline Access of care & & & & & \\
\hline $\begin{array}{l}\text { Telephone vs hospital f-up } \\
\text { Technical competence }\end{array}$ & $75.8(16.1)$ & $74.7(15.3)$ & 3.10 & -.13 to 6.32 & 0.060 \\
\hline Telephone vs hospital f-up & $75.1(16.7)$ & $74.1(18.0)$ & 1.76 & -1.91 to 5.42 & 0.347 \\
\hline
\end{tabular}

a Positive differences imply a higher level of satisfaction in the telephone group.

\section{DISCUSSION}

To improve follow-up care after breast cancer, feedback from patients on satisfaction is important since it provides information on the quality of care received. Furthermore, patients may comply better with a specific follow-up strategy when they are satisfied with their care and follow-up setting. Insight into patient satisfaction when evaluating telephone follow-up was therefore an important aspect of our RCT.

The results of this study show that nurse-led telephone follow-up after breast cancer may well be an appropriate alternative to hospital follow-up. Patient satisfaction scores at 12 months after treatment were high in all subscales of the PSQ III for both nurseled telephone and hospital follow-up. No meaningful differences were found between the two types of follow-up in scores for general satisfaction, satisfaction with interpersonal aspects, satisfaction with technical competences of staff, and access of care. Since equivalence was seen as a positive outcome in this study, it was important to carefully analyze protocol violators and perform both intention to treat and per protocol analyses (Jones et al., 1996). Both types of analyses showed almost identical results. This was expected since the 30 patients who violated the protocol in the telephone group did not 
significantly differ from other participants of this group regarding age, level of education, treatment received and baseline satisfaction scores.

To our knowledge, this was the first study to evaluate the multidimensional concept of patient satisfaction with nurse-led telephone follow-up specifically aimed at the first year after breast cancer treatment. It is also one of the few studies that used a validated questionnaire to measure patient satisfaction (Sitzia, 1999). Although the PSQ III was not specifically developed to measure satisfaction with follow-up care and response bias is a documented problem of the questionnaire, the Dutch version was validated among breast cancer patients after treatment and satisfaction and response bias scores found in our study were similar to the norm scores found by Hagedoorn et al. (2003). Furthermore, the pragmatic nature of the RCT led to the inclusion of a broad range of patients regarding age, treatment and location of treatment, including a sample representative of breast cancer patients in the Netherlands (Kiemeney et al., 2008).

In general, the positive findings in this study were similar to findings reported in other studies investigating nurse-led telephone follow-up (Beaver et al., 2009b; Brown et al., 2002; Hagedoorn et al., 2003; Montgomery et al., 2008a). Even though the results are difficult to compare, there is a strong trend towards acceptability, feasibility, and good patient satisfaction scores for telephone follow-up. The recent study by Beaver et al. (2009b) is most comparable to our study. In their randomized clinical trial $(n=374)$, a structured telephone intervention was developed and nurses received four half day training sessions, similar to our study. Patient satisfaction was evaluated at the beginning, middle, and end of the trial, by asking participants if they were satisfied with information received and whether the appointment had been helpful in dealing with their concerns. It was found that patient satisfaction was significantly higher for telephone follow-up compared to hospital follow-up at the middle and at the end of the trial. Women were recruited between 0.5 and 106 months after the end of treatment and remained in the trial for a mean of 24 months. In our study women entered immediately after treatment and remained in the trial for 12 months. Thus, the studies can be seen as complementary; both provide evidence that telephone follow-up can be appropriate for patients in different phases after breast cancer treatment (Beaver et al., 2009b).

Several explanations can be hypothesized for the high satisfaction scores with nurseled telephone follow-up. First of all, telephone follow-up was performed by a specialized breast care nurse, most often a nurse familiar to the patient from the time of diagnosis and treatment. It was expected that patients felt comfortable expressing emotions and concerns to this nurse (Pennery \& Mallet, 2000). The breast care nurses were also specifically trained to meet information and psychological needs. The follow-up may take up to 20 minutes and was done by open discussion, offering the patient the opportunity to discuss issues they were most concerned with. Furthermore, nurse-led telephone followup provided continuity to the patient. In general, the same nurse provided the telephone follow-up for a patient, which is different from hospital follow-up where patients were seen by the medical oncologist, radiation oncologist, surgeon or breast care nurse. 
Despite positive results, the conclusion that nurse-led telephone follow-up provides equal satisfaction compared to hospital follow-up must be made carefully, taking into account several possible limitations of this study. First, of eligible patients $64 \%$ declined participation in the randomized trial. This might be due to a lack of knowledge about the purpose and effectiveness of follow-up (de Bock et al., 2004b; Montgomery et al., 2008b). Moreover, the fact that patients were informed about the usual care (hospital follow-up) before participation may have also negatively influenced the participation rate, since patients generally have a preference for what they know best, the so-called 'status quo bias' (Salkeld et al., 2000). Hence, patient education on follow-up will need special attention in future trials or when implementing telephone follow-up. Nevertheless, the relatively low participation rate of the RCT may have an impact on the generalizabilty of our results. It specifically influenced this patient satisfaction study, since patients who chose to participate in the RCT may have had either no strong preference for a follow-up strategy, or positive expectations of the interventions. Thus, patients may have been somewhat uncritical of the care provided, or prior expectations about follow-up may have influenced expressed satisfaction (Linder-Pelz, 1982; Williams, 1994). In other words, patients may have expressed satisfaction no matter what care was provided. It is unclear whether and if so, to what extent, the sample selection has influenced our results. Additionally, patients who had developed a recurrence or metastatic disease in the study period were lost to follow-up. Hence, the analysis included a sample of patients who remained disease-free in the first year after treatment. It may be speculated that these patients will generally show high satisfaction scores.

Second, one in five patients randomized to telephone follow-up did not receive telephone follow-up according to protocol, which may in itself be seen as evidence that the two follow-up strategies were not entirely equal in terms of preference and feasibility. Indeed, ten patients requested to receive hospital follow-up directly after having been randomized for telephone follow-up. However, most other protocol violations were related to logistic difficulties or health-related problems (e.g. the patient needed to visit the hospital for a complaint, but was unintentionally not re-entered in the telephone follow-up). Moreover, even though hospital follow-up was better adhered to in the trial and generally preferred beforehand, per protocol analyses showed high satisfaction scores for telephone followup, equal to patients who followed hospital follow-up.

Third, it must be recognized that all patients received some follow-up in the hospital, also the patients randomized to telephone follow-up, which may have contributed to the fact that no differences between the two forms of follow-up were found.

Finally, other outcomes besides patient satisfaction are relevant when exploring alternative follow-up strategies for breast cancer, such as the effectiveness in terms of health-related quality of life, emotional functioning, feelings of anxiety and costs. These outcome measures will be assessed before implementation of telephone follow-up. 


\section{CONCLUSION}

This study showed that regular telephone contact with a breast care nurse and a oneyear mammography combined with a hospital visit was equal to traditional hospital follow-up visits, in terms of general satisfaction, and satisfaction with regard to technical competences of staff, access of care and interpersonal aspects. It is concluded that nurse-led telephone follow-up in the first year after breast cancer treatment may be an appropriate and acceptable alternative to hospital follow-up. 


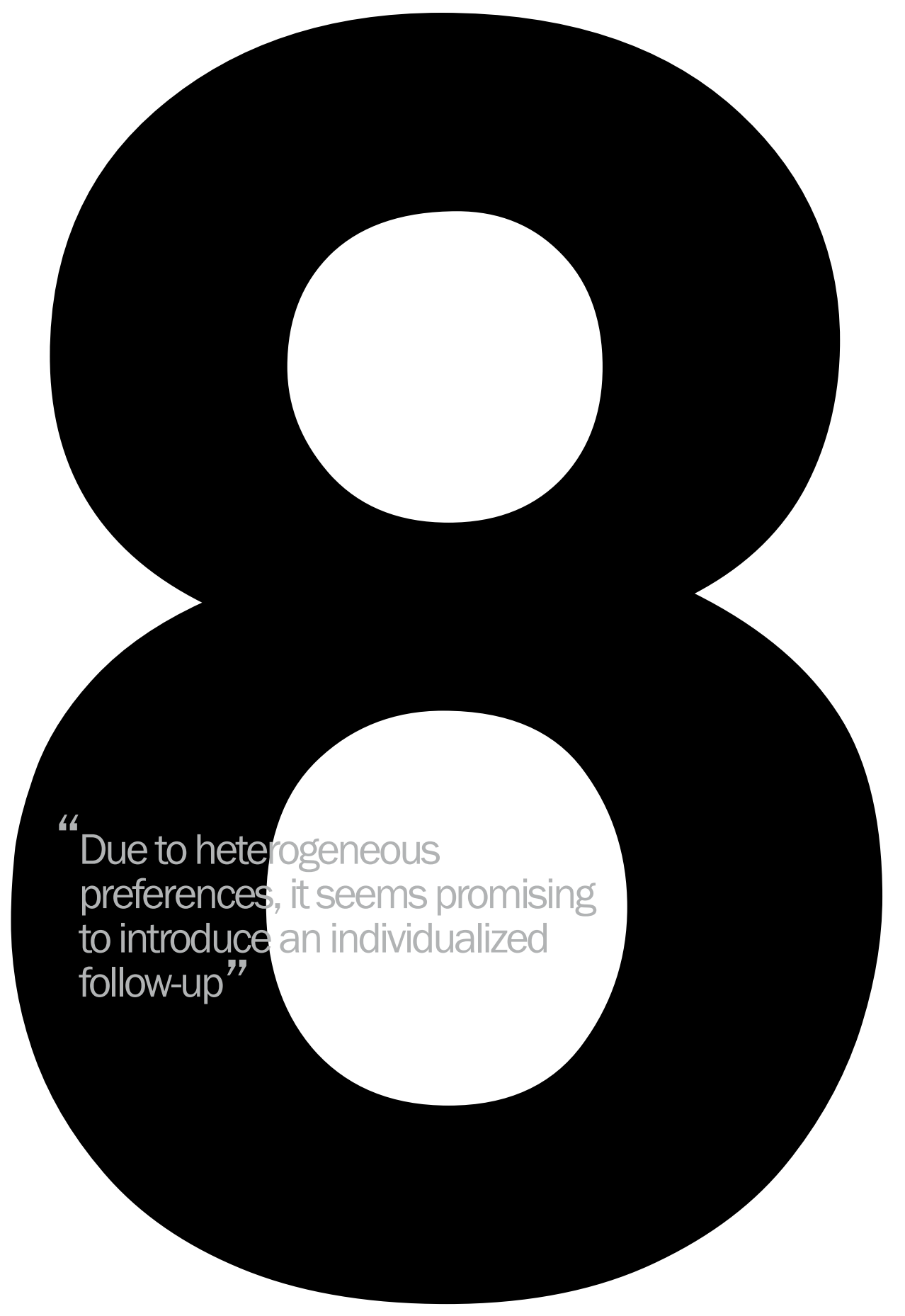




\section{Follow-up after treatment for breast cancer: One strategy fits all? An investigation of patient preferences using a discrete choice experiment}

KIMMAN ML, DELLAERT BGC, BOERSMA L, LAMBIN Ph, DIRKSEN CD

ACTA ONCOL 2010: 49(3): 328-337

\section{ABSTRACT}

[Introduction] Clinical guidelines for the follow-up after breast cancer recommend frequent outpatient clinic visits to be examined for a possible recurrence or a second primary breast tumor, and to receive information and psychosocial support. However, needs and preferences for follow-up may differ between patients, raising the question whether the current 'one size fits all' approach is appropriate. This study explored patients' preferences for follow-up. [Patients and methods] A discrete choice experiment survey with 16 choice tasks was filled out by 331 breast cancer patients. Each choice task consisted of two hypothetical follow-up scenarios for the first year after treatment, described by levels of the following characteristics; attendance at an educational group program, frequency of visits, waiting time, contact mode, and type of health care provider. [Results] The health care provider and contact mode were the most important characteristics of follow-up to patients. The medical specialist was the most preferred to perform the follow-up, but a combination of the medical specialist and breast care nurse alternating was also acceptable to patients. Face-toface contact was strongly preferred to telephone contact. Follow-up visits every three months were preferred over visits every four, six, or twelve months. Heterogeneity in preference between patients was strong, especially for the health care provider and attendance at an educational group program. Age, education, and previous experience with follow-up characteristics influenced preferences, but treatment modality did not. [Conclusions] The results of this study show that overall patient satisfaction would not differ significantly if patients have follow-up by medical specialist and breast care nurse alternating compared to follow-up by a medical specialist only. Furthermore, we found heterogeneity in preferences for most attributes, indicating that one strategy does not fit all. Individualized follow-up seems to offer the potential for significant increases in patient satisfaction. 


\section{INTRODUCTION}

Clinical guidelines for follow-up after curative treatment for breast cancer recommend that women attend frequent follow-up clinic visits, to be examined for possible local or regional recurrence or a second primary breast tumor, and to receive information and psychosocial support (Pestalozzi et al., 2005). However, clinical outcomes are not affected by frequency, intensity, and type of follow-up (GIVIO, 1994; Grunfeld et al., 2006; Koinberg et al., 2004; Rosselli Del Turco et al., 1994), and there is doubt whether short outpatient clinic visits are appropriate to provide psychosocial support (Allen, 2002; Pennery \& Mallet, 2000). Hence, a more individualized follow-up, in which patient preferences are taken into account, is being proposed (Grunfeld, 2009a). Particularly in the first year after treatment, when scheduled follow-up visits are most frequent, a shared decision making process and more flexible follow-up may increase patient satisfaction, adherence to the strategy, and ultimately quality of life (Joosten et al., 2008).

Several studies have investigated patient preferences regarding breast cancer followup using cross-sectional surveys. De Bock et al. (2004b) analyzed the needs of women who participated in a routine follow-up program $(n=116)$. More than half of the patients preferred lifetime follow-up, twice a year, performed by the medical specialist. Furthermore, younger age was related to a greater need for information during follow-up. Montgomery et al. (2008b) found that women $(n=79)$ expected some form of follow-up, but there was no consistency regarding the frequency. Renton et al. (2002) concluded that, although women were generally satisfied with current hospital follow-up, they would accept changes. Results of the study suggest that if services were reorganized, women would prefer involvement of specialist nurses in follow-up to that of their general practitioner. Hence, women do expect to receive some form of follow-up after breast cancer, but preferences for patient education, length, frequency, and provider of follow-up can vary considerably. Additionally, results from several clinical trials show that patients who have experienced alternative follow-up strategies, e.g. by a breast care nurse (Koinberg et al., 2004), by a general practitioner (Grunfeld et al., 2006), less frequent follow-up (Brown et al., 2002), or follow-up by telephone (Beaver et al., 2009b) were equally satisfied as patients who received traditional hospital follow-up.

This paper deals with women's preferences for the follow-up after breast cancer treatment, including most alternative strategies that have been proposed in the literature, using a discrete choice experiment (DCE). A DCE is a survey methodology capable of establishing preferences, which is grounded in economic theory (Lancaster, 1966; McFadden, 1974), and has an advantage over traditional satisfaction questionnaires, in that it enables the researcher to measure strength of preferences for different characteristics of follow-up and the trade-offs made between them. DCEs are found to be a valid and reliable approach to elicit preferences in a health care context (Bryan et al., 2000) and are recognized as a useful tool for medical decision making (Lancsar \& Louviere, 2008; Ryan \& Gerard, 2003). 
In a DCE respondents are asked to choose the preferred alternative from a set of two or more hypothetical scenarios. The scenarios are described by key characteristics ('attributes') of follow-up, such as the health care provider. The respondents' evaluation of a scenario depends on the levels of the attributes (for example, a nurse, medical specialist, or general practitioner). The relative importance of attributes and levels to respondents and the trade-offs made between them, can be assessed by asking respondents to make choices in multiple scenarios with different levels of attributes. A detailed explanation of the methodology applied in health care has been described by Lanscar and Louviere (2008), and Ryan and Gerard (2003).

To our knowledge, this is the first study that aims to measure the strength of preferences for several characteristics of breast cancer follow-up using a discrete choice experiment. We also explore the variety in patient preferences (i.e. 'preference heterogeneity') which ultimately allows us to explore optimal ways of providing individual-level follow-up to patients.

\section{PATIENTS AND METHODS}

\section{Patients}

This study was performed among Dutch breast cancer patients who finished curative treatment between June 2006 and December 2007. Data collection took place between May and July 2008. Of 557 eligible patients from five participating hospitals, 359 agreed to participate and subsequently received a survey with the discrete choice experiment. Completed surveys from 331 patients were used for the analysis (response rate of 59\%). Respondents' characteristics can be found in table 1 (page 92). The average age was 58 years (range 34 to 83 ) and the mean time since finalizing breast cancer treatment was 14 months (range 4 to 24 months). The study was approved by the Medical Ethical Committee of the Maastricht University Medical Center.

\section{Methods}

A discrete choice experiment survey was used. The survey comprised two sections: the DCE (16 hypothetical choice tasks) and a section with questions regarding background information of the respondent.

A crucial stage of DCE design is the identification of the key characteristics (attributes) that describe the scenarios in the choice tasks, and their levels (Lancsar \& Louviere, 2008; Ryan, 1999). The choice of these attributes and levels was based on a review of the literature (Kimman et al., 2007a), local policy initiatives (Kimman et al., 2007b), and expert opinion. Attributes were included if they represented common current practice in follow-up or relevant potential initiatives. Table 2 (page 94) provides the attributes and levels used in the choice tasks.

The combination of attributes and levels in this study resulted in $\left(4^{3} \times 2^{2}=\right) 256$ possible scenarios (a 'full factorial design'). Since presenting all 256 scenarios to respondents 
would be too burdensome, only a subset of the scenarios, a fractional factorial design, was used. With a 32-profile fractional factorial design we were able to estimate main effects and two-way interactions between contact mode and all other attributes. Subsequently, to construct choice tasks, a fold-over of the design was created. A fold-over takes the original design, shifts the attribute levels (e.g. medical specialist becomes breast care nurse, every three months becomes every four months, etc), and adds this fold-over design as extra scenarios to the original design, creating choice tasks. The design ensured orthogonality (the absence of multicollinearity between attributes) and was balanced in terms of the number of times each level of an attribute was seen (Street et al., 2005). A maximum of 16 choice tasks per respondents was considered feasible, hence two surveys each with 16 choice tasks were created by blocking the design. This means that each respondent faces only a subset of choice situations from the fractional factorial design. Blocks were determined based on an additional orthogonal column to the design with two levels. Respondents were randomly assigned to one of the two blocks. An example of a choice task is shown in figure 1.

Table 1. Characteristics of the 331 respondents of the discrete choice experiment

\begin{tabular}{|c|c|}
\hline Category & No. respondents (\%) \\
\hline \multicolumn{2}{|l|}{ Age } \\
\hline$<60$ year & $145(43.8)$ \\
\hline$\geq 60$ year & $186(56.2)$ \\
\hline \multicolumn{2}{|l|}{ Living with a partner } \\
\hline Living alone or single parent & $79(23.9)$ \\
\hline Living with partner (and children) & $247(74.6)$ \\
\hline Other & $5(1.5)$ \\
\hline \multicolumn{2}{|l|}{ Education } \\
\hline No education, primary school, lower education & $111(33.5)$ \\
\hline Secondary education & $134(40.5)$ \\
\hline Higher education & $86(25.0)$ \\
\hline \multicolumn{2}{|l|}{ Treatment modality } \\
\hline Surgery & $48(14.5)$ \\
\hline Surgery and radiotherapy & $128(38.7)$ \\
\hline Surgery and chemotherapy & $58(17.5)$ \\
\hline Surgery and radiotherapy and chemotherapy & $96(29.0)$ \\
\hline Unknown & $1(0.3)$ \\
\hline \multicolumn{2}{|l|}{ Hormonal therapy } \\
\hline Yes & $126(38.1)$ \\
\hline No & $204(61.6)$ \\
\hline Unknown & $1(0.3)$ \\
\hline \multicolumn{2}{|l|}{ Experience with follow-up } \\
\hline Follow-up by a breast care nurse & $37(11.2)$ \\
\hline Follow-up by a medical specialist & $220(66.5)$ \\
\hline Combination of breast care nurse and medical specialist & $74(22.3)$ \\
\hline Participation in educational group program & $27(8.2)$ \\
\hline Experience with telephone follow-up & $24(7.3)$ \\
\hline
\end{tabular}


Important! Before choosing between scenario A and B, please be aware of the following:

Regardless of the scenario you will have an annual mammography combined with a visit (with physical examination) to the medical specialist. Also, you can always make additional appointments whenever you feel the need. Disease-free and overall survival are identical for both scenarios.

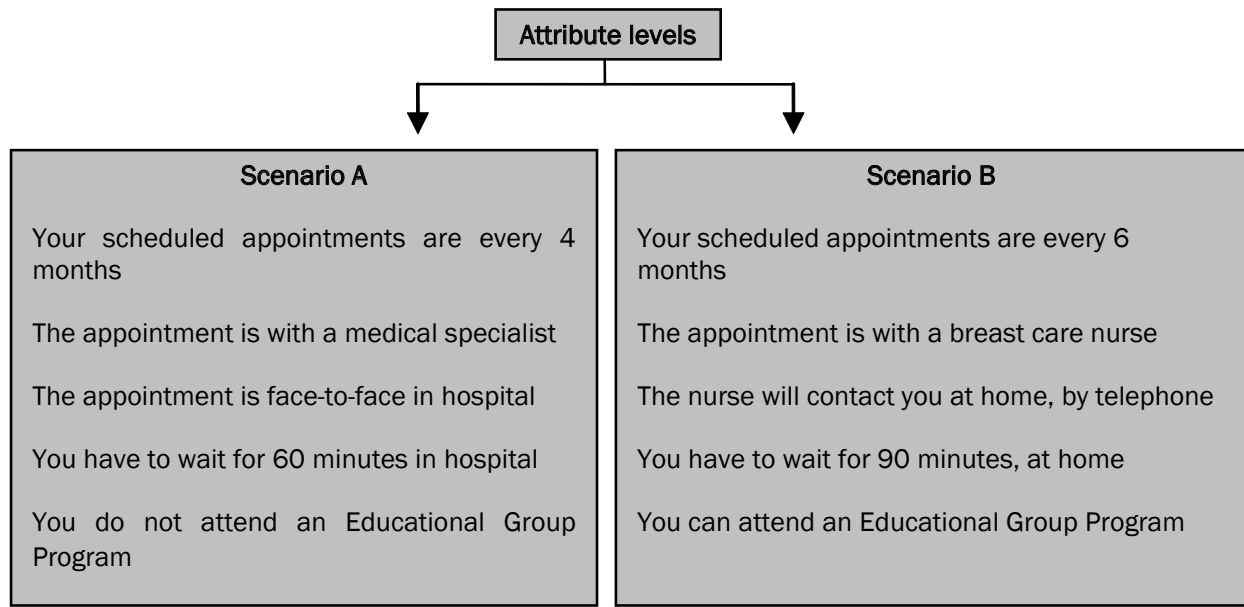

\section{Choice}

For the first year after treatment, would you prefer scenario A or B?

Figure 1. Structure of a discrete choice task. Scenarios varied systematically over 32 choice tasks.

The survey started with a short introduction on why the study was performed and an outline of the purpose and effectiveness of follow-up after breast cancer. A comprehensive description of each attribute and its level to clarify their meaning and implications to respondents followed. Importantly, every choice task started with information on the effectiveness of follow-up, the annual mammography, and the possibility to make additional appointments (see figure 1). To be able to investigate whether patients preferred what they themselves had experienced (Salkeld et al., 2000), respondents were asked to describe the follow-up strategy they had received in their first year after treatment. Additionally, background information on education level, living with a partner or alone, and received treatment modality was retrieved. The survey was pilot tested $(n=10)$ to check for any problems in interpretation and face validity, which led to minor changes to the text and layout. 


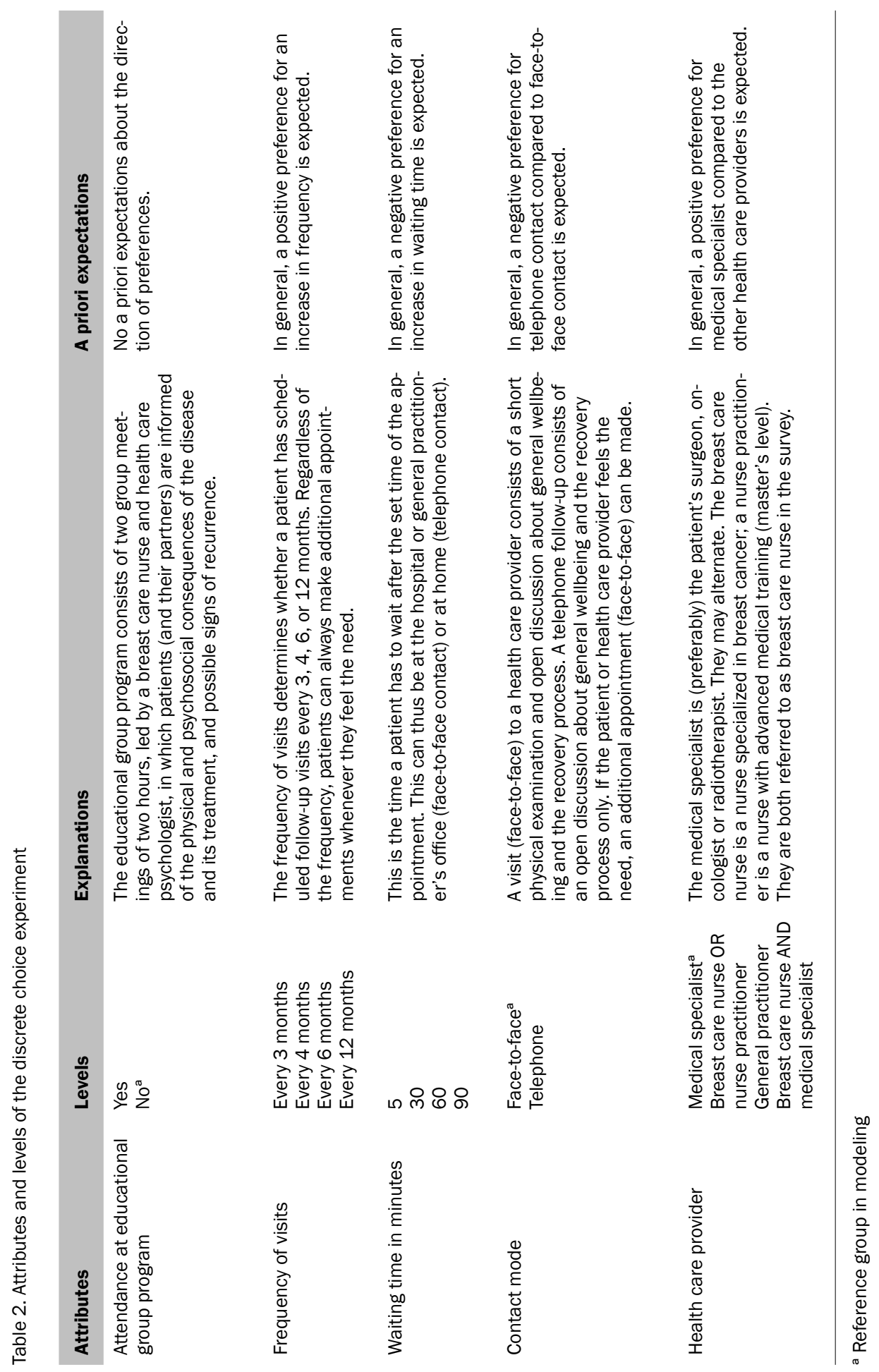




\section{Establishing preferences}

All eligible patients were contacted by telephone by independent employees of the Center for Data and Information Management of the Maastricht University to ask for participation. When informed consent was obtained, respondents received the postal survey. Respondents were asked to fill out the survey at home. Contact details of the researcher were given for any questions or clarification. At a later time, answers to the completed survey were collected by telephone and entered into a database. NLOGIT 3.0 software package (Econometric Software Inc.) was used to analyze the data.

Random parameter logit (RPL) modeling was used to estimate a choice model and specify a utility function (Kjaer \& Gyrd-Hansen, 2008; Train, 2003). All probabilistic choice models are characterized by the following equation:

$$
\mathrm{U}_{\text {in }}=\mathrm{V}_{\text {in }}+\varepsilon_{\text {in }}
$$

where $V_{\text {in }}=g_{n}\left(X_{i}\right)$ is the systematic utility component that depends on the attributes of the alternative $\left(\mathrm{X}_{\mathrm{i}}\right)$ and the indivual's specific tastes, and $\varepsilon_{\text {in }}$ is an error component. Assuming that a respondent can choose between two alternatives, $i$ and $j$, then the probability that alternative $\mathrm{i}$ is chosen is given by:

$$
P_{\text {in }}=\operatorname{Prob}\left(U_{\text {in }}>U_{j n}\right)=\operatorname{Prob}\left(V_{\text {in }}+\varepsilon_{\text {in }}>V_{\text {in }}+\varepsilon_{\text {jn }}\right)=\operatorname{Prob}\left(V_{\text {in }}-V_{\text {jn }}>\varepsilon_{\text {jn }}-\varepsilon_{\text {in }}\right) \text { if } i
$$

is not equal to $\mathrm{j}$.

This generates the well known standard logit specification if the error terms are assumed to follow independent and identical Gumbel distributions:

$$
P_{\text {in }}=\exp \left(V_{\text {in }}\right) /\left(\exp \left(V_{\text {in }}\right)+\exp \left(V_{\text {in }}\right)\right)
$$

In the random parameters logit model the systematic utility component is expressed as a function of the alternative's attributes and an individual specific vector of preference parameters as follows:

$$
V_{\text {in }}=\beta_{n}{ }^{\prime} X_{i} \text { with } \beta_{n}=\left(\beta+v_{n}\right),
$$

where $\beta$ constitutes the vector of average preferences of the population for each attribute and $v_{n}$ the individual's specific preference components which take on a normal distribution.

Our utility function was of the following form:

$\mathrm{U}_{\mathrm{ijt}}=\beta_{0}+v_{0 \mathrm{i}}+\left(\beta_{1}+v_{1 \mathrm{i}}\right) *$ Educational group program $\mathrm{j}+\left(\beta_{2}+v_{2 \mathrm{i}}\right) *$ Frequency $_{\mathrm{j}}+\left(\beta_{2}+\right.$ $\left.v_{3 i}\right) *\left(\text { Frequency }_{j}\right)^{2}+\left(\beta_{4}+v_{4 i}\right) *$ Waiting time $_{j}+\left(\beta_{5}+v_{5 i}\right) *$ Telephone contact $_{j}+\left(\beta_{6}+v_{6 i}\right) *$ Breast care nurse ${ }_{j}+\left(\beta_{7}+v_{7 i}\right) *$ General practitioner ${ }_{j}+\left(\beta_{8}+v_{8 \mathrm{i}}\right) *$ Medical specialist / Breast care nurse $_{j}+\varepsilon_{\mathrm{ijt}}$

For a detailed explanation see Appendix 1. In short, we investigated changes in utility (i.e. preference or satisfaction) when a level of an attribute changed. The parameter estimates $\left(\beta_{1}-\beta_{8}\right)$ of the utility function describe the magnitude of these utility changes, and indicated 
the relative importance of an attribute. A statistically significant parameter estimate indicates that the attribute is indeed important to respondents. However, statistical nonsignificance of a parameter of an attribute should not be seen as necessarily meaning 'not relevant' for each respondent since, due to heterogeneity in preferences, for some respondents the attribute may still influence their decisions. A positive or negative sign indicates that an attribute-level is either preferred or not preferred compared to the base level of the attribute. Preference heterogeneity was explored by examining the significance of standard deviations of error components around the mean parameter estimates. Respondents' characteristics were incorporated into the model as interaction terms with the attributes of the DCE to explore possible sources of preference heterogeneity.

Internal validity, i.e. the extent to which results are consistent with a priori expectations, was tested by examining the signs and significances of parameter estimates. We expected all attributes to have a significant influence on preferences for follow-up. Furthermore, it was expected that, in general, frequency would have a positive sign in the utility function and waiting time to have a negative sign. Since face-to-face follow-up by the medical specialist is common in current clinical practice, we expected a general preference for the medical specialist over the other health care professionals, and a general preference for face-to-face contact over telephone contact (hence a negative sign for the parameter estimate of telephone contact). No specific assumptions were made about preferences for the educational group program. Regarding two-way interactions, we were specifically interested in the interaction between waiting time and contact mode. It was a likely possibility that there were differences in preferences for waiting time between waiting time at the hospital (face-to-face contact) and waiting time at home (telephone contact). Additionally, a consistency check (two identical choice tasks) was incorporated in the survey, and respondents were asked to report whether they experienced difficulties understanding the choice tasks and choosing between the alternatives.

\section{RESULTS}

\section{Validity of responses}

Most respondents (74\%) found the choice tasks clear or very clear, $16 \%$ were indifferent, and $10 \%$ found them unclear. Of the 331 respondents, 310 (94\%) passed the consistency check. The Kappa statistic $(\mathrm{K})$ for the identical choice tasks was 0.72 , representing a good agreement. Following recent recommendations (Lancsar \& Louviere, 2008), data from all respondents $(n=331)$ were used for the main analysis. However, a secondary analysis was performed with a calibrated sample of respondents who found the choice tasks (very) clear $(n=244)$. This analysis showed similar parameter estimates and model fit (data not shown). Furthermore, most parameter estimates were significant $(p<0.05)$, and the parameter estimates of the attributes were in the expected direction, providing evidence of internal validity. In addition, the constant term was not significant, indicating there was no general preference for alternative 'A' over alternative ' $B$ ', when all attributes and levels 
were the same. Contrary to expectations, the two-way interaction between contact mode and waiting time was not significant ( $p=0.687$ ). Finally, an adjusted R-square of 0.369 in this study represented a good fit of the model, indicating that respondents made choices in a systematic way, based on changes in the scenarios (Hensher et al., 2005).

\section{Preferences, relative importance of attributes and trade-offs}

Table 3 shows the results of the discrete choice experiment. The two-way interactions between contact mode and other attributes did not improve the model fit nor did they alter conclusions, therefore only results of the main effects model are reported. The main effects model showed that in general, for type of health care professional, followup by the medical specialist was preferred to follow-up only by a breast care nurse or general practitioner, as indicated by their negative parameter estimates. The parameter estimates for medical specialist and breast care nurse alternating and the educational group program (EGP) were not significant. This may indicate that, in general, a switch from the medical specialist performing the follow-up to alternating between the medical specialist and a breast care nurse, and whether or not an EGP is part of the follow-up, would not negatively (or positively) affect utility. Alternatively, it could mean that there is heterogeneity in preferences for these attributes.

As expected, more frequent visits per year and reducing waiting time also had a positive impact on utility. However, the significant negative frequency ${ }^{2}$ variable indicates that there is a linear and quadratic effect for frequency. Hence, marginal utility of more frequent follow-up visits decreased considerably when frequency increased. There was a large decrease in utility for a change from face-to-face contact to telephone contact.

The relative importance of the attributes and their levels were determined by calculating part-worth utilities (Ryan, 1999); their ranking is shown in table 3. Overall, contact mode (face-to-face contact) and health care provider (medical specialist versus general practitioner) were the most important attributes of follow-up for respondents, while waiting time, the educational group program, and whether the health care provider was a breast care nurse or a medical specialist were least important.

Inputting parameter estimates and levels of attributes in the utility function provides information on how respondents were willing to trade between levels of attributes. For example, a change from the medical specialist to the breast care nurse performing the follow-up would result in a utility loss of 0.46 , when all other attribute levels remain equal. However, this can be compensated by an increase in the frequency of visits. Utility for follow-up visits every six months (i.e. twice a year) is $(\beta * \text { frequency }+\beta * \text { (frequency })^{2}=$ $1.497 * 2-0.212 * 2^{2}=$ ) 2.146 and for follow-up visits every four months (i.e. 3 times a year) $\left(1.497 * 3-0.212 * 3^{2}=\right) 2.583$. Hence, increasing the frequency results in a utility gain of 0.44 , which could compensate for the loss of utility due to a change in health care provider. 
Table 3. Results for the random parameter logit model ( $n=331)$ with main effects only, results for the model with interactions with experience, and relative importance of attributes. Results presented are mean parameter estimates, standard deviations and p-values. $\mathrm{EGP}=$ educational group program, $\mathrm{BCN}=\mathrm{breast}$ care nurse.

\begin{tabular}{|c|c|c|c|c|c|c|}
\hline & & \multicolumn{2}{|c|}{ Main effects model ${ }^{a}$} & \multicolumn{2}{|c|}{$\begin{array}{l}\text { Experience } \\
\text { interaction model }^{b}\end{array}$} & \multirow{2}{*}{$\begin{array}{l}\text { Relative } \\
\text { impor- } \\
\text { tance }^{c}\end{array}$} \\
\hline & & $\begin{array}{l}\text { Parameter } \\
\text { estimate }\end{array}$ & p-value & $\begin{array}{l}\text { Parameter } \\
\text { estimate }\end{array}$ & p-value & \\
\hline \multicolumn{7}{|c|}{ Random parameters in utility function ${ }^{d}$} \\
\hline \multirow[t]{2}{*}{ EGP 1} & Mean & -0.138 & 0.088 & -0.212 & 0.011 & \\
\hline & Standard dev & 1.171 & $<0.001$ & 1.117 & $<0.001$ & \\
\hline \multirow[t]{2}{*}{ Frequency of visits } & Mean & 1.497 & $<0.001$ & 1.541 & $<0.001$ & $0.13(3)$ \\
\hline & Standard dev & 0.429 & $<0.001$ & 0.463 & $<0.001$ & \\
\hline \multirow[t]{2}{*}{ Telephone contact ${ }^{2}$} & Mean & -2.027 & $<0.001$ & -2.279 & $<0.001$ & $0.36(2)$ \\
\hline & Standard dev & 1.533 & $<0.001$ & 1.505 & $<0.001$ & \\
\hline \multirow[t]{2}{*}{ Breast care nurse $(\mathrm{BCN})^{3}$} & Mean & -0.463 & $<0.001$ & -0.617 & $<0.001$ & $0.08(4)$ \\
\hline & Standard dev & 1.057 & $<0.001$ & 1.126 & $<0.001$ & \\
\hline \multirow[t]{2}{*}{ General practitioner ${ }^{3}$} & Mean & -2.124 & $<0.001$ & -2.195 & $<0.001$ & $0.38(1)$ \\
\hline & Standard dev & 1.749 & $<0.001$ & 1.899 & $<0.001$ & \\
\hline \multirow[t]{2}{*}{ Waiting time } & Mean & -0.007 & $<0.001$ & -0.008 & $<0.001$ & $0.05(5)$ \\
\hline & Standard dev & 0.007 & 0.007 & 0.008 & $<0.001$ & \\
\hline \multicolumn{7}{|c|}{ Non-random parameters in utility function e } \\
\hline Constant & Mean & -0.075 & 0.109 & -0.076 & 0.114 & \\
\hline Frequency ${ }^{2}$ & Mean & -0.212 & $<0.001$ & -0.215 & $<0.001$ & \\
\hline Medical specialist/ BCN ${ }^{3}$ & Mean & 0.137 & 0.110 & 0.124 & 0.159 & \\
\hline Experience * EGP & Mean & -0.138 & 0.088 & 0.698 & 0.011 & \\
\hline Experience * tel f-up & Mean & 1.171 & $<0.001$ & 2.004 & $<0.001$ & \\
\hline Experience * $\mathrm{BCN}$ & Mean & 1.497 & $<0.001$ & 1.101 & $<0.001$ & \\
\hline LL(model) & & -2303.323 & -2259.043 & & & \\
\hline Adjusted pseudo $\mathrm{R}^{2}$ & & 0.369 & 0.377 & & & \\
\hline $\mathrm{N}$ & & 5296 & 5296 & & & \\
\hline
\end{tabular}

a The main effects model assumed no interactions were present.

' The experience interaction model incorporated respondents' previous experience with follow-up.

c The relative importance of attributes and their levels was determined by calculating the coefficient range, which is the difference between the smallest (negative) part-worth utility and the largest part-worth utility within the attribute levels, and dividing it by the sum of the coefficient ranges for all attributes and levels. ${ }^{\mathrm{d}}$ Attributes and levels were assigned to be normally distributed random parameters.

e When random parameters were found to have an insignificant standard deviation for their distribution, they were included as a nonrandom parameter in the model estimations.

${ }^{1}$ Refers to the attribute 'attendance at educational group program'. Relative to 'no attendance at educational group program'.

2 Refers to the attribute 'contact mode'. Relative to 'face-to-face contact'.

${ }^{3}$ Refers to the attribute 'health care provider'. Relative to 'medical specialist'. 


\section{Exploring preference heterogeneity}

Our estimation results demonstrated that there was a strong heterogeneity in patient preferences for follow-up characteristics (table 3). To gain insight into the strength of preference heterogeneity, individual parameter estimates were calculated of the 331 respondents. By counting the number of patients with a positive parameter estimate for a specific attribute-level, the percentage of the sample that preferred that attribute-level compared to the base level was calculated. Especially, preferences for follow-up by a breast care nurse and participation in the EGP differed greatly among respondents (table 4). For the total study population, a change from the medical specialist to a breast care nurse resulted in lower utility, but $28 \%$ of patients would nevertheless prefer this kind of follow-up. Also, the possibility to attend the EGP did not seem to influence utility, but indeed $47 \%$ of the study population preferred participation to no participation.

To identify which patients might share specific preferences, the following interaction terms were incorporated into the model: chemotherapy, radiotherapy, hormonal therapy, living with a partner, older than 60 years, and high level of education. Only age and education level influenced preferences. Follow-up by a medical specialist was valued more positively by older than younger respondents (<60 years). Furthermore, older respondents and those with a low level of education were less likely to choose a scenario that included attending the EGP. Respondents with a higher education level positively valued an EGP in the follow-up. Though interactions between age, education and some attributes were significant, collectively they only minimally improved the fit of the model. Hence, much of the heterogeneity remained unexplained.

Table 4. Preference heterogeneity explored by individual parameter estimates $(n=331)$ : results presented are the number of respondents with a positive parameter estimate and negative parameter estimate for attributes of the discrete choice experiment (\% of sample).

\begin{tabular}{lll} 
& $\begin{array}{l}\text { Positive parameter } \\
\text { estimate (\% of sample) }\end{array}$ & $\begin{array}{l}\text { Negative parameter } \\
\text { estimate (\% of sample) }\end{array}$ \\
$\begin{array}{l}\text { Educational group program } \\
\text { (vs no educational group program) }\end{array}$ & $156(47)$ & $175(53)$ \\
$\begin{array}{l}\text { Increase in frequency of visits } \\
\begin{array}{l}\text { Telephone contact } \\
\text { (vs face-to-face contact) }\end{array}\end{array}$ & $328(99)$ & $3(1)$ \\
$\begin{array}{l}\text { Follow-up by a breast care nurse } \\
\text { (vs medical specialist) }\end{array}$ & $17(5)$ & $314(95)$ \\
$\begin{array}{l}\text { Follow-up by a general practitioner } \\
\text { (vs medical specialist) }\end{array}$ & $93(28)$ & $238(72)$ \\
$\begin{array}{l}\text { Increase in waiting time } \\
\text { (1) }\end{array}$ & $0(0)$ & $321(97)$ \\
\hline
\end{tabular}




\section{Influence of previous experience on preferences}

Our study population included some respondents who had experienced telephone followup, nurse-led follow-up, and the EGP. Hence, we were able to create interactions between having experienced an attribute and the choice data for this attribute. The results are presented in table 3, and showed that respondents who had attended the EGP had a significantly higher, and positive, parameter estimate for this attribute than those who had not. The same was true for respondents who had experienced follow-up by a breast care nurse. The parameter estimate for telephone contact was considerably higher for respondents who had experienced telephone follow-up, but telephone follow-up was still not preferred to face-to-face contact. These results indicated that there was indeed a tendency to prefer what one had personally experienced; however, the subgroups were small, and preference heterogeneity was still significant when previous experience was accounted for.

\section{DISCUSSION}

\section{Patient preferences}

To our knowledge this is the first study to investigate patients' preferences for breast cancer follow-up using discrete choice experimentation. Most patients preferred the medical specialist to perform the follow-up, but an alternating combination of the medical specialist and a breast care nurse was also acceptable to patients, and one in four patients preferred the follow-up to be performed solely by a breast care nurse. Face-to-face contact was much preferred to telephone contact. Furthermore, utility increased with an increase in the frequency of visits and when waiting time was reduced. Contact mode and type of health care provider were the most important characteristics of follow-up to patients.

The finding that alternating between the medical specialist and breast care nurse is acceptable to patients is not surprising. There is evidence that emotional concerns are more easily expressed to a nurse (Pennery \& Mallet, 2000), while seeing a medical specialist provides a feeling of security (de Bock et al., 2004b). Hence, alternating between both health care providers seems a good alternative (Renton et al., 2002). In line with our findings, other studies have also found that the general practitioner was generally not preferred (Mao et al., 2009; Renton et al., 2002). A study conducted among American breast cancer patients revealed that patients had concerns about the communication between oncologists and general practitioners (Mao et al., 2009). Similar feelings among Dutch breast cancer patients may have caused the strong disutility for follow-up with a general practitioner, since in the Netherlands general practitioners only have a marginal role in breast cancer treatment and follow-up. A dislike for telephone follow-up is not surprising as it is unknown to patients, and may seem unsafe when patients still believe that frequent clinical examination to detect a relapse is an essential part of the followup (de Bock et al., 2004b; Montgomery et al., 2008b). Even though we tried to provide information on the purpose and effectiveness of follow-up, we did not provide detailed 
information on the value of clinical examinations.

Finally, the need for information has also been studied before, and in general information needs varied greatly (Montgomery et al., 2008b). This is in line with our finding that preferences for participation in the EGP were very heterogeneous. Furthermore, in our study younger patients ( $<60$ years) and patients with a higher level of education had a stronger preferences for the EGP than older patients (and less educated), which is similar to other findings (de Bock et al., 2004b; Montgomery et al., 2008b). De Bock et al. (2004b) did find that higher information needs were associated with receiving adjuvant hormonal therapy or chemotherapy, but we could not find evidence for the influence of treatment modality on preferences for the educational group program.

Importantly, when interpreting these results, we have to realize that people tend to prefer what they know best or have experienced (Salkeld et al., 2000), which was confirmed in our study. Hence, preferences for participation in an unknown educational group program (EGP) and telephone contact or follow-up by a general practitioner might have been underestimated, while the importance of follow-up by the medical specialist and face-toface contact might have been overestimated. Moreover, this tendency to prefer what one has experienced might be part of the explanation why results of randomized clinical trials are in contrast with our findings. A very recent study by Beaver et al. (2009b) found that telephone follow-up was acceptable to most patients, it significantly improved satisfaction, and it produced no excess anxiety compared with hospital follow-up. A large randomized clinical trial by Grunfeld et al. (2006) investigating follow-up with the general practitioner also found high satisfaction rates for this type of follow-up. However, in both studies 40$45 \%$ of the approached patients refused participation. Although reasons for refusal can vary, it may indicate that these alternative follow-up strategies were not acceptable to all patients, which is more in line with our findings.

\section{Implications for clinical practice}

The results of this study raise several possibilities regarding the introduction of innovations in breast cancer follow-up. One possibility is to introduce a follow-up strategy in which the medical specialist and breast care nurse (alternating) provide the follow-up. With an increasing population of breast cancer survivors, a less specialized strategy could already create possibilities for scarce specialized knowledge to be used elsewhere. It can also be speculatively argued that, due to the tendency to favor the follow-up strategy that one has personally experienced, simply implementing the follow-up strategy that is the least costly might be efficient. However, our results show that even when respondents had experience with the breast care nurse or telephone follow-up, some still preferred alternative strategies. Rather, due to the heterogeneous preferences, it seems promising to introduce an individualized follow-up to maximize utility.

Identifying patient characteristics that predict preferences for follow-up would be of use in implementing flexible follow-up. Interestingly, our study could not identify any objective patient characteristics that were strongly associated with preferences for follow- 
up attributes. Age and education interacted with preferences to some extent, but other individual-level factors such as personality type, lifestyle, risk aversion, anxiety, and perceived quality of life after treatment may further explain respondents' preference differences. Better still, as preferences were hard to predict based on socio-economic or treatment characteristics, a self-selection procedure after treatment may well be the most effective option to classify patients for different follow-up strategies. Future research could evaluate such individualized follow-up in terms of satisfaction and its economic impact. It is expected that individualized follow-up could decrease health care costs, because of preferences for less specialized follow-up (e.g. a follow-up visit to a nurse is likely to cost less than a visit to the medical specialist), telephone contact, and less frequent followup. However, individualized follow-up could also have a potential negative effect on costs because of loss of economies of scale and possibly increased use of health care services outside the hospital.

\section{Limitations}

Some limitations of DCEs in their application to this study must be mentioned. First, an important consideration in designing a DCE is the selection of attributes and levels. These were based on a literature review, local policy initiatives and expert opinions. Focus groups and other qualitative research methods could be part of future research to investigate whether important attributes were missing.

Second, though a response rate of $59 \%$ is generally high, the validity of the findings may have been affected by respondent selection bias, and it is unclear how this would affect preference estimates for follow-up strategies. However, sample characteristics were promising in the sense that respondents with different treatment modalities, treating hospitals, age, and educational level were well represented in the sample and matched those of the study population (Ernst et al., 2004).

Third, a key element in the use of random parameter logit modeling is the assumption regarding the distribution of each of the random parameters. In our main analysis all attributes and the constant term were assigned as normally distributed random parameters to allow for a high flexibility in possible heterogeneity. To ensure that our results were robust for distributions, we performed a secondary analysis, in which the attribute levels general practitioner, telephone contact, and frequency, were assigned a lognormal distribution (based on the observed relatively limited heterogeneity in preferences in these attributes, see table 3). This led to highly similar effects and a marginal improvement in model fit (adjusted R-square of 0.372), but restricted respondents' preferences to be either all positive or all negative for a given attribute level. Since an important aim of our study was to explore preference heterogeneity, allowing respondents to have either a negative or positive preference for an attribute level, the analysis results with normal distributions for the random parameters were used for the purpose of this paper.

Fourth, respondents had to make a so-called forced choice, and were not offered an 'optout' or 'status-quo' alternative in the experiment. It is argued that the exclusion of an opt- 
out or status-quo option is a violation of the underlying welfare measures of the economic experiment, since it makes it impossible to estimate the value of doing nothing, which may be real market behavior (Bateman et al., 2002). However, we felt that the inclusion of such an option had more disadvantages than advantages. A potential problem of an opt-out alternative is that if the respondent feels that the choice task is cognitively demanding, it will be tempting to simplify it by choosing the opt-out or status-quo option, even though this would not provide the highest utility. Moreover, even though in clinical practice women could choose not to have follow-up, there is no evidence in the literature that this is common in clinical practice. Adding a status-quo alternative would have been a possibility, but also raised two concerns. First of all we were cautious for the 'status-quo bias', the tendency to choose what respondents know best (Salkeld et al., 2000), since respondents were already experienced with breast cancer follow-up. Second, the statusquo alternative differed among respondents, causing econometric and interpretation difficulties.

Finally, levels of anxiety or psychological distress of participants were not measured in this study, while it could be speculated that anxiety and psychological distress influence patient preferences and adherence to a follow-up strategy. Future research should incorporate such individual-level factors, and when appropriate, they might be taken into account in the decision-making process for a follow-up strategy. This is in line with recommendations from governmental and professional organizations that cancer patients be screened routinely for the presence of heightened psychological distress (NCCN, 1999; NICE, 2004).

\section{CONCLUSION}

On balance, DCEs have great potential for medical decision making by offering a valid and reliable approach to provide estimates of the most valuable characteristics of follow-up to patients. We have investigated how changes in breast cancer follow-up affect patients' preferences or utility. The results of this study contribute to the literature findings that overall patient satisfaction would not differ significantly if patients have follow-up by medical specialist and breast care nurse alternating compared to follow-up by a medical specialist only. Furthermore, we found heterogeneity in preferences for most attributes, indicating that one strategy does not fit all. Since preferences are difficult to predict, the introduction of individualized follow-up based on self-selection seems to be a promising method to maximize benefits in terms of patient satisfaction. Subsequently, it would be interesting to investigate the economic impact of such an individualized follow-up and whether patients who choose their own follow-up are indeed more satisfied than patients assigned to a standard follow-up strategy. 


\section{Appendix 1.}

An RPL model takes account of preference heterogeneity by placing a distribution around some, or all, parameters. Furthermore, it allows one to capture the fact that multiple observations are obtained from a single respondent, which is most often the case in a DCE survey where each respondent typically evaluates multiple choice sets. In the RPL model, the dependent variable was whether or not a scenario (A or B) was selected by the patient in a given choice set, while the independent variables were the attributes. The attribute "frequency" was coded in terms of a linear and quadratic effect (i.e., frequency ${ }^{2}$ ) to investigate whether the incremental utility of a greater number of contacts decreased when frequency increased. All attributes and the constant term were initially assigned as normally distributed random parameters. A parameter with an insignificant standard deviation for its distribution, was re-specified as a nonrandom parameter, and the model was re-estimated (Hensher et al., 2005). The RPL model was estimated for the total sample, using 500 Halton draws.

The function to be estimated was of the following form:

$\mathrm{U}_{\mathrm{ijt}}=\beta_{0}+v_{0 \mathrm{i}}+\left(\beta_{1}+v_{1 \mathrm{i}}\right) *$ Educational group program $_{\mathrm{j}}+\left(\beta_{2}+v_{2 \mathrm{i}}\right) *$ Frequency $_{\mathrm{j}}+\left(\beta_{2}+\right.$ $\left.v_{3 i}\right) *\left(\text { Frequency }_{j}\right)^{2}+\left(\beta_{4}+v_{4 i}\right) *$ Waiting time $_{j}+\left(\beta_{5}+v_{5 i}\right) *$ Telephone contact $_{j}+\left(\beta_{6}+v_{6 i}\right) *$ Breast care nurse $_{j}+\left(\beta_{7}+v_{7 i}\right) *$ General practitioner $_{j}+\left(\beta_{8}+v_{8 i}\right) * M S / B C N_{j}+\varepsilon_{i j t}$

where;

$\mathrm{U}_{\mathrm{ijt}}$ is individual i's utility (i.e., satisfaction) associated with a specific follow-up scenario j in choice observation $\mathrm{t}$.

$\beta_{0}$ represents the constant term and shows the general preference for scenario A over $B$ when all attributes and levels are the same.

$\beta_{1}-\beta_{8}$ are the parameter estimates of the model that indicate the importance of each attribute as it occurs in scenario $\mathrm{j}$. The sign of a parameter estimate reflects whether the attribute has a positive or negative effect on utility. "EGP" is a dummy variable for EGP being available or not, "telephone contact" is a dummy variable for telephone vs. face-toface contact, and "BCN" (breast care nurse), "GP”, and “MS/BCN" (alternating between medical specialist and breast care nurse) are dummy variables reflecting the different types of follow-up contact persons, with "MS" (medical specialist) as a base level. The base levels reflect current practice levels in the Netherlands.

The different $v_{i} s$ correspond to the individual specific error terms for every parameter which are constant across all observations for the individual and are assumed to be independently normal distributed, and $\varepsilon_{\mathrm{ijt}}$ is an error term which captures any remaining unobserved error and is assumed to be IID gumbel distributed among observations (Hensher et al., 2005).

To test moderating effects of age, education, treatment modality and hormonal therapy we extended the model with the product variables of the corresponding individual characteristics and the variables manipulated in the DCE. 



$$
9
$$


General discussion
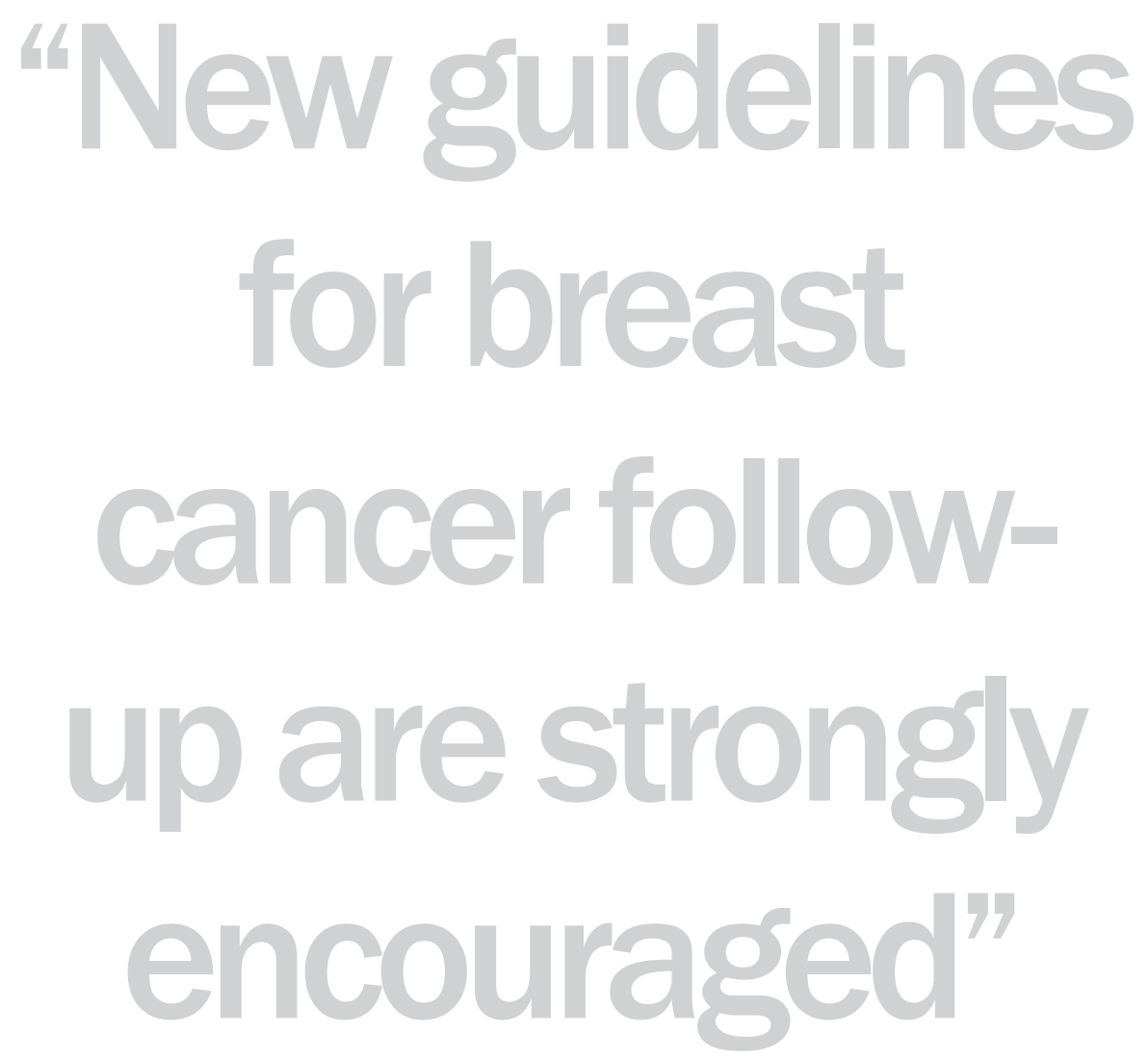


\section{INTRODUCTION}

The value of follow-up of breast cancer patients remains controversial despite almost two decades of research. Studies have shown that different models of follow-up can be equally effective, measured by a variety of outcomes. Ultimately, however, these studies have not been found to be sufficiently convincing and researchers continue the search for the best model. This thesis was based on five years of research concerning breast cancer followup and focused on the first year after completion of primary treatment. Two interventions were developed and tested in a randomized controlled setting. The first intervention, nurse-led telephone follow-up, aimed to replace hospital follow-up visits without a loss of health-quality of life (HRQoL) or increase in anxiety. The second intervention, a short educational group program (EGP), aimed to provide information and improve HRQoL. An extensive economic evaluation was performed alongside the randomized controlled trial (RCT) to provide detailed insight into costs and consequences of four follow-up strategies and determine the most cost-effective strategy. Furthermore, patients' satisfaction with nurse-led telephone follow-up was examined, and the strength of preferences for several characteristics of follow-up was explored using discrete choice experimentation.

In this final chapter, the main research findings are briefly summarized. Subsequently, both strengths and methodological concerns regarding the research are discussed, followed by recommendations and suggestions for future research.

\section{SUMMARY OF MAIN FINDINGS}

The research performed showed that most hospital clinic visits in the first year after treatment may well be replaced by nurse-led telephone follow-up without loss of HRQoL or increase in feelings of anxiety. Additionally, replacing hospital follow-up with nurseled telephone follow-up did not affect role and emotional functioning, and feelings of control. Furthermore, patients were equally satisfied when follow-up was performed by telephone or in hospital. Hence, nurse-led telephone follow-up seems an appropriate way to reduce clinic visits and represents an accepted alternative strategy. An educational group program in addition to follow-up may be appropriate to address information needs, but it did not unequivocally affect positive HRQoL outcomes. Importantly, results of the economic evaluation showed that nurse-led telephone follow-up plus EGP was costeffective compared to three alternative follow-up strategies. Nevertheless, it was found that preferences for follow-up were very heterogeneous, suggesting that individualized follow-up may increase satisfaction.

\section{STRENGTHS OF THE RESEARCH}

The MaCare trial was an RCT designed as a pragmatic trial. Pragmatic trials measure the beneficial effects of an intervention in a setting closely reflecting real practice, as opposed to an ideal research situation (Roland \& Torgerson, 1998). These trials are specifically 
interesting to provide evidence for health care providers and policy makers for policy decisions. Interventions are compared to usual care and selection criteria are kept to a minimum to reflect variations between patients that occur in real practice. Hence, the MaCare trial included a broad range of patients, from seven hospitals in the Netherlands, with all stages of non-metastatic breast cancer (stage I, II or III), and treated with either surgery alone, or in combination with chemotherapy and/or radiotherapy. The first study arm, hospital follow-up, represented care as usual in the participating hospitals.

The $2 \times 2$ factorial design of the MaCare trial allowed for efficient testing of two interventions, reducing the impact of the trial on resources, doctors, and patients. Furthermore, a high quality economic evaluation was performed alongside the RCT. The economic evaluation was performed according to published international guidelines for trial-based economic evaluations (Earnshaw \& Lewis, 2008), and closely reflected the resource use that would be observed in practice. Four alternative strategies were compared, which is relatively uncommon and overcomes the often proposed disadvantage of trial-based economic evaluations that they represent only a limited form of analysis by comparing few alternatives (Sculpher et al., 2006). Importantly, by using micro costing to value resources and presenting tables with resource use and cost prices, detailed information was provided on follow-up costs, enabling case-by-case calculations for the purpose of generalizability (Sculpher et al., 2004).

At the individual clinical decision level, women's preferences for different characteristics of follow-up, including most alternative strategies that have been proposed in the literature, were explored using a discrete choice experiment (DCE). This survey methodology is grounded in economic theory (Lancaster, 1966; McFadden, 1974) and enables the researcher to measure heterogeneity in preferences, as well as the strength of preferences for different characteristics of follow-up and the trade-offs made between them. DCEs are found to be a valid and reliable approach to elicit preferences in a health care context (Bryan et al., 2000) and are recognized as a useful tool for medical decision making (Lancsar \& Louviere, 2008; Ryan \& Gerard, 2003).

\section{METHODOLOGICAL CONSIDERATIONS}

Some methodological considerations regarding the studies performed in this thesis must be addressed.

First, in the CEA performed alongside the RCT, the generally recommended EQ-5D was used for the measurement and calculation of health state values (utilities) (Earnshaw \& Lewis, 2008). However, concern has been raised whether the generic EQ-5D is sensitive enough to pick up (clinical) changes in certain patient groups (Eurich et al., 2006; Krahn et al., 2007; van de Willige et al., 2005). Results of the study into the responsiveness of the EQ-5D (chapter 5) showed that the EQ-5D was able to capture both improvements and deteriorations in HRQoL of breast cancer patients after treatment. However, according 
to Cohen's benchmarks for effect sizes (Cohen, 1988), the EQ-5D was only responsive to moderate and large changes in health, but not to small changes. Nevertheless, cutoff points for effect sizes or discriminative ability are less relevant in the context of economic evaluations. A small difference in the EQ-5D might still be meaningful when additional costs for such a change in HRQoL are very low. Therefore, it was concluded that the EQ-5D was an appropriate measure for the economic evaluation. A limitation of this responsiveness study was that responsiveness was investigated using a single anchor, while ideally multiple anchors should be used to investigate the responsiveness of an instrument (Guyatt et al., 2002). For example, clinical expert opinion on a patient's health status or a second measure of quality of life such as the SF-6D (Brazier et al., 2002) or SF-36 (Ware \& Sherbourne, 1992) could have been collected in order to better assess responsiveness.

Second, of eligible patients, $64 \%$ declined participation in the RCT. Reasons for refusal varied; some patients preferred face-to-face contact to telephone contact (26.9\%), others did not like the idea of participation in the educational group program (16.5\%), 24.6\% were too tired, disliked trials in general or felt uncomfortable completing questionnaires, and for $32 \%$ the reason for refusal was unknown. The fact that patients were informed about the usual care (hospital follow-up) before participation may have negatively influenced the participation rate, since patients generally have a preference for what they know best, the so-called 'status quo bias' (Salkeld et al., 2000). The relatively low participation rate of the RCT may have an impact on the generalizabilty of our results. It specifically influenced our patient satisfaction study, since patients who chose to participate in the RCT may have had either no strong preference for a follow-up strategy, or positive expectations of the interventions. Thus, patients may have been somewhat uncritical of the care provided, or prior expectations about follow-up may have influenced expressed satisfaction (LinderPelz, 1982; Williams, 1994). In other words, patients may have expressed satisfaction no matter what care was provided.

It is unclear whether and if so, to what extent, the sample selection has influenced our study results. Nevertheless, women who participated in the trial did not differ from nonparticipants with respect to education, marital status, HRQoL or other psychological outcome measures. Furthermore, the pragmatic nature of the trial led to the inclusion of a broad range of patients regarding age, treatment and location of treatment, and as a result our study population was indeed representative of breast cancer patients in the Netherlands (Kiemeney et al., 2008).

Finally, to gain insight into patient preferences for non-health aspects of follow-up, a patient preference study was performed in the form of a discrete choice experiment (DCE) (Hensher et al., 2005). When interpreting results of a DCE, it is important to realize that people tend to prefer what they know best or have experienced (Salkeld et al., 2000). As a consequence, the choice of the study population can therefore have a significant 
impact on the results. The DCE was performed among women who had been treated for breast cancer and who were between six months and two years in follow-up. An advantage of these respondents was that they provided relatively informed preferences for most attributes. However, the majority of the sample did not have experience with the EGP, telephone contact and follow-up by the general practitioner (GP). Hence, preferences for these characteristics of follow-up relied on the information provided during the experiment. While all attributes and their levels were extensively described in the survey, it is unclear whether this information was carefully read and understood. Due to the inexperience with the EGP, telephone contact and follow-up by the GP, preferences for these attribute levels may have been underestimated. Additionally, the importance of follow-up by the medical specialist and face-to-face contact might have been overestimated, since this form of follow-up was experienced by most patients. An analysis on a small subsample of patients with experience with nurse-led telephone follow-up and EGP indeed showed that they were more satisfied with these forms of follow-up compared to inexperienced patients.

\section{RECOMMENDATIONS FOR CLINICAL PRACTICE AND POLICY}

Just recently, in 2009, the new NICE (National Institute for Clinical Excellence) guidance for early and locally advanced breast cancer was developed and was a long awaited replacement of the 2002 guidance. Importantly, a key recommendation of the new guidelines is that patients should have a choice in their method of follow-up (Harnett et al., 2009). Furthermore, in 2007, the Health Council of the Netherlands published a report on cancer follow-up, with its main recommendation being the implementation of an individualized 'aftercare plan' for each patient within five years after publication (Gezondheidsraad, 2007). This aftercare plan must contain written information about the physical and psychological impact of the disease and its treatment, about the desirability and form of follow-up, and about other ongoing points for attention.

However, incorporating individual preferences in treatment decisions may create conflict. The treatment recommended to an individual patient to meet preferences may differ substantially from the most cost-effective treatment (Brazier et al., 2009). This conflict is likely to arise in the case of breast cancer follow-up. While the CEA showed that nurse-led telephone follow-up plus EGP would be the most cost-effective strategy, the DCE showed that hospital follow-up was preferred to telephone follow-up and that preferences for health care provider and the EGP were very heterogeneous. Moreover, new guidelines for follow-up should be as much evidence based as possible, and the effectiveness and costeffectiveness of individualized follow-up remain to be investigated. Individualized followup may, somewhat unexpectedly, not be cost-effective compared to usual care if it has a negative effect on costs because of loss of economies of scale or increased use of health care services outside the hospital. It also needs to be explored whether the implementation of a different strategy for each patient is feasible in clinical practice. Therefore, instead of implementing individualized follow-up it is suggested to first investigate the feasibility and 
cost-effectiveness of individualized follow-up.

At this time, based on the results reported in this thesis, it is recommended to reduce the frequent follow-up visits in the first 12 months after treatment to one follow-up visit to the medical specialist, including physical examination and a mammography. Additionally, the three-monthly hospital visits can be replaced by nurse-led telephone follow-up and an EGP. Furthermore, it is recommended that the breast care nurse takes a central role in the follow-up. A well-designed nurse-led follow-up can increase continuity and devote more time to patient education. Moreover, it allows for the reallocation of medical specialist time from follow-up to diagnosis and treatment and utilizes the skills of specially trained nurses in an optimal way. For patients who are very anxious after completion of treatment it is recommended to provide hospital follow-up and the EGP, instead of telephone followup, since a reduced follow-up strategy for these patients had contrary effects on their resource use.

New guidelines for breast cancer follow-up are strongly encouraged, since they can promote efficient and discourage inefficient follow-up. However, guidelines do not automatically translate into clinical practice, especially when the new guideline requires better collaboration among disciplines, changes in patients' behavior or changes in the organization of care (Grol \& Grimshaw, 2003; Grol et al., 2007). Since patient preferences seem to be in favor of hospital follow-up, it is extremely important to readily invest in the implementation and dissemination of the new guideline. Insight into barriers and facilitators can help to design the most effective implementation strategy (Grol, 1997). In our opinion, successful implementation of this new guideline requires primarily a paradigm shift among doctors and patients. As long as there is a false idea among medical specialists that patients need constant reassurance and a long-standing belief that considering costs is unseemly and unethical, the implementation of a new guideline will likely be unsuccessful. At the same time, preferences for hospital follow-up may be a result of false expectations about the purpose and effectiveness of follow-up among patients (de Bock et al., 2004b). Hence, information about risks of recurrence, early detection, survival estimates and aims of follow-up, are crucial for the success of a new (reduced) follow-up strategy. Our EGP may be appropriate to address patients' information needs and educate the patient about the purpose and effectiveness of follow-up. Also, an individual aftercare plan, as proposed by the Health Council, describing (among other things) the purpose of follow-up and when and where the follow-up and EGP take place, can enhance successful implementation and adherence to the new strategy. Furthermore, educational materials for health care professionals involved in follow-up as well as outreach visits may be used for the implementation of the new guideline (Grol \& Grimshaw, 2003).

As a final remark it must be noted that new developments regarding diagnostics and therapeutics may lead to changes in the follow-up. The follow-up should be as much evidence based as possible. Therefore, every five years, the follow-up model should be reconsidered, taking into account new scientific insights, and adjust guidelines accordingly. 


\section{AREAS FOR FUTURE RESEARCH}

Since our pragmatic trial has shown cost-effectiveness of nurse-led telephone follow-up with EGP, this is likely to be cost-effective in real-life, since the circumstances approximate real-life settings. However, guidelines are not always adhered to by both doctors and patients, and the cost-effectiveness of actual implementation of nurse-led telephone follow-up plus EGP may be less than estimated with the pragmatic trial if implementation is very costly and adherence poor (Mason et al., 2001). Ideally, future research should examine possible barriers and facilitators for implementation before the development of strategies to implement the new strategy (Grol, 1997). Subsequently, several scenarios with varying costs of implementation and doctor and patient adherence could be explored with regard to the cost-effectiveness of implementation (Mason et al., 2001).

Results of our preference study suggested that individualized follow-up could enhance patient satisfaction. Future research could investigate whether individualized follow-up is indeed feasible in clinical practice. In order to respond to individual preferences, the medical specialist will need to move from a generally paternalistic approach towards a shared model for the choice of follow-up (Coulter, 1999). A decision-support tool enhances this shared-decision making process, and can possibly improve satisfaction and compliance to the chosen follow-up strategy. This decision-support tool should incorporate a range of characteristics of the patient, including tumor characteristics, treatments received, and preferences for follow-up. We are currently exploring whether such a decision-support tool can be developed for breast cancer follow-up and implemented in clinical practice. Subsequently, it is important to study whether patients who were actively involved in the choice of follow-up strategy, indeed show improved outcomes in terms of satisfaction and quality of life, and how individualized follow-up impacts on cost-effectiveness.

Finally, since this research has shown that nurse-led telephone follow-up is good value for money, it seems promising to study whether this follow-up strategy would be transferable to other cancer patient groups. 

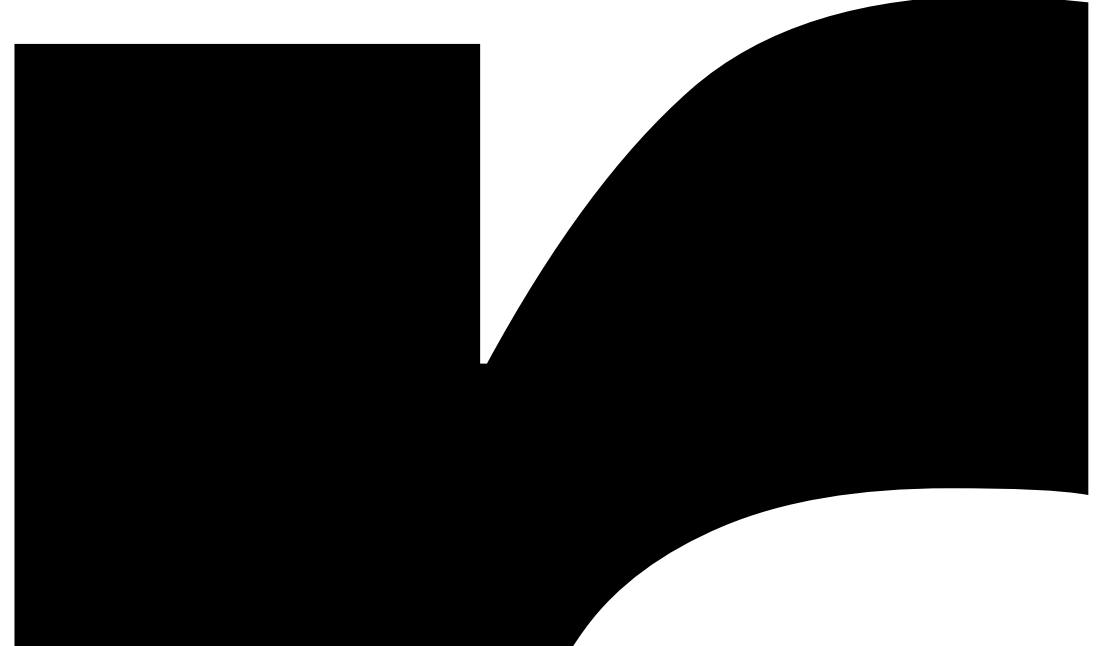


\section{References}

Aaronson NK, Ahmedzai S, Bergman B, Bullinger M, Cull A, Duez NJ, et al. The European Organization for Research and Treatment of Cancer QLQ-C30: a quality-of-life instrument for use in international clinical trials in oncology. J Natl Cancer Inst 1993;85(5):365-76.

Adewuyi-Dalton R, Ziebland S, Grunfeld E, Hall A. Patients' views of routine hospital follow-up: a qualitative study of women with breast cancer in remission. Psychooncology 1998;7(5):436-9.

Allen A. The meaning of the breast cancer follow-up experience for the women who attend. Eur J Oncol Nurs 2002;6(3):155-61.

Anell A, Norinder A. Health outcome measures used in cost-effectiveness studies: a review of original articles published between 1986 and 1996. Health Policy 2000;51(2):87-99.

Antoni MH, Lehman JM, Kilbourn KM, Boyers AE, Culver JL, Alferi SM, et al. Cognitive-behavioral stress management intervention decreases the prevalence of depression and enhances benefit finding among women under treatment for early-stage breast cancer. Health Psychol 2001;20(1):20-32.

Arndt V, Merx H, Stegmaier C, Ziegler H, Brenner H. Persistence of restrictions in quality of life from the first to the third year after diagnosis in women with breast cancer. J Clin Oncol 2005;23(22):4945-53.

Arndt V, Merx H, Sturmer T, Stegmaier C, Ziegler H, Brenner H. Age-specific detriments to quality of life among breast cancer patients one year after diagnosis. Eur J Cancer 2004;40(5):673-80.

Bartelink H, Horiot JC, Poortmans P, Struikmans H, Van den Bogaert W, Barillot I, et al. Recurrence rates after treatment of breast cancer with standard radiotherapy with or without additional radiation. $\mathrm{N}$ Engl J Med 2001;345(19):1378-87.

Bateman IJ, Carson RT, Day B, Hanemann M, Hanley N, Hett T, et al. Economic Evaluation with Stated Preference Techniques, A Manual. 1 ed. Cheltenham: Edward Elgar Publishing Limited; 2002.

Beaver K, Hollingworth W, McDonald R, Dunn G, Tysver-Robinson D, Thomson L, et al. Economic evaluation of a randomized clinical trial of hospital versus telephone follow-up after treatment for breast cancer. The British journal of surgery 2009a;96(12):1406-1415.

Beaver K, Luker KA. Follow-up in breast cancer clinics: reassuring for patients rather than detecting recurrence. Psychooncology 2005;14(2):94-101.

Beaver K, Tysver-Robinson D, Campbell M, Twomey M, Williamson S, Hindley A, et al. Comparing hospital and telephone follow-up after treatment for breast cancer: randomised equivalence trial. BMJ 2009b;338:a3147.

Bennett B, Goldstein D, Lloyd A, Davenport T, Hickie I. Fatigue and psychological distress-exploring the relationship in women treated for breast cancer. Eur J Cancer 2004;40(11):1689-95. 
Bottomley A, Aaronson NK. International perspective on health-related quality-of-life research in cancer clinical trials: the European Organisation for Research and Treatment of Cancer experience. J Clin Oncol 2007;25(32):5082-6.

Brada M. Is there a need to follow-up cancer patients? Eur J Cancer 1995;31A(5):655-7.

Brazier J, Roberts J, Deverill M. The estimation of a preference-based measure of health from the SF-36. Health Econ 2002;21(2):271-92.

Brazier J, Roberts J, Tsuchiya A, Busschbach J. A comparison of the EQ-5D and SF-6D across seven patient groups. Health Econ 2004;13(9):873-84.

Brazier JE, Dixon S, Ratcliffe J. The role of patient preferences in cost-effectiveness analysis: a conflict of values? PharmacoEconomics 2009;27(9):705-12.

Briggs AH, O'Brien BJ. The death of cost-minimization analysis? Health Econ 2001;10(2):179-84.

Briggs AH, Wonderling DE, Mooney CZ. Pulling cost-effectiveness analysis up by its bootstraps: a nonparametric approach to confidence interval estimation. Health Econ 1997;6(4):327-340.

Brown L, Payne S, Royle G. Patient initiated follow up of breast cancer. Psychooncology 2002;11(4):34655.

Bryan S, Gold L, Sheldon R, Buxton M. Preference measurement using conjoint methods: an empirical investigation of reliability. Health Econ 2000;9(5):385-95.

Bureau of Statistics Netherlands. (2008). “http://statline.cbs.nl accessed November 2009.” Statline.

Clark MM, Bostwick JM, Rummans TA. Group and individual treatment strategies for distress in cancer patients. Mayo Clin Proc 2003;78(12):1538-43.

Cohen J. Statistical Power Analysis for Behavioral Science. 2nd ed. Hilsdale, NJ: Lawrence Earlbaum Associates; 1988.

Comprehensive Cancer Centers. "Breast cancer incidence in the Netherlands 2006." www.ikcnet.nl

Coulter A. Paternalism or partnership? Patients have grown up-and there's no going back. BMJ 1999;319(7212):719-20.

Cox K, Wilson E. Follow-up for people with cancer: nurse-led services and telephone interventions. J Adv Nurs 2003;43(1):51-61.

Crow R, Gage H, Hampson S, Hart J, Kimber A, Storey L, et al. The measurement of satisfaction with healthcare: implications for practice from a systematic review of the literature. Health technology assessment 2002;6(32):1-244.

de Bock GH, Bonnema J, van der Hage J, Kievit J, van de Velde CJ. Effectiveness of routine visits and routine tests in detecting isolated locoregional recurrences after treatment for early-stage invasive breast cancer: a meta-analysis and systematic review. J Clin Oncol 2004a;22(19):4010-8.

de Bock GH, Bonnema J, Zwaan RE, van de Velde CJ, Kievit J, Stiggelbout AM. Patient's needs and preferences in routine follow-up after treatment for breast cancer. Br J Cancer 2004b;90(6):1144-50. 
Deconinck E, Miadi-Fargier H, Pen CL, Brice P. Cost effectiveness of rituximab maintenance therapy in follicular lymphoma: long-term economic evaluation. PharmacoEconomics 2010;28(1):35-46.

Dewar J. Follow up in breast cancer. BMJ 1995;310(6981):685-6.

Dewar JA, Kerr GR. Value of routine follow up of women treated for early carcinoma of the breast. Br Med J (Clin Res Ed) 1985;291(6507):1464-7.

Dolan P. Modeling valuations for EuroQol health states. Med Care 1997;35(11):1095-108.

Drummond MF, Sculpher MJ, Torrance GW, O'Brien BJ, Stoddart GL. Methods for the economic evaluation of health care programmes. 3rd ed. Oxford: Oxford University Press; 2005.

Earnshaw J, Lewis G. NICE Guide to the Methods of Technology Appraisal: Pharmaceutical Industry Perspective. PharmacoEconomics 2008;26(9):725-7.

Ernst MF, Voogd AC, Coebergh JW, Roukema JA. Breast carcinoma diagnosis, treatment, and prognosis before and after the introduction of mass mammographic screening. Cancer 2004;100(7):1337-1344.

ESMO. ESMO Minimum Clinical Recommendations for diagnosis, adjuvant treatment and follow-up of primary breast cancer. Ann Oncol 2001;12(8):1047-8.

Eurich DT, Johnson JA, Reid KJ, Spertus JA. Assessing responsiveness of generic and specific health related quality of life measures in heart failure. Health Qual Life Outcomes 2006;4:89.

EuroQoL-Group. EuroQol--a new facility for the measurement of health-related quality of life. The EuroQol Group. Health Policy 1990;16(3):199-208.

Fayers P, Bottomley A. Quality of life research within the EORTC-the EORTC QLQ-C30. European Organisation for Research and Treatment of Cancer. Eur J Cancer 2002;38 Suppl 4:S125-33.

Fenwick E, Byford S. A guide to cost-effectiveness acceptability curves. Br J Psychiatry 2005;187:106-8.

Fenwick E, O’Brien B, Briggs A. Cost-effectiveness acceptability curves--facts, fallacies and frequently asked questions. Health Econ 2004;13(5):405-415.

Freedman GM, Li T, Anderson PR, Nicolaou N, Konski A. Health states of women after conservative surgery and radiation for breast cancer. Breast Cancer Res Treat 2010;121(2):519-26.

Ganz PA, Coscarelli A, Fred C, Kahn B, Polinsky ML, Petersen L. Breast cancer survivors: psychosocial concerns and quality of life. Breast Cancer Res Treat 1996;38(2):183-199.

Gezondheidsraad. Nacontrole in de oncologie. Doelen onderscheiden, inhoud onderbouwen. Den Haag; 2007. Report No.: 2007/10.

Gijsen BCM, Koppejan-Rensenbrink AG. Herstel \& Balans, groepsrevalidatie in de oncologie. Tijdschrift Kanker 2003;27(2):36-9.

GIVIO. Impact of follow-up testing on survival and health-related quality of life in breast cancer patients. A multicenter randomized controlled trial. The GIVIO Investigators. JAMA 1994;271(20):1587-92. 
Gold MR, Siegel JE, Russell LB, Weinstein MC. Cost-effectiveness in health and medicine. New York: Oxford University Press; 1996.

Goodwin PJ, Black JT, Bordeleau L, Ganz PA. Health-related quality-of-life measurement in randomized clinical trials in breast cancer--taking stock. Journal of the National Cancer Institute 2003;95(4):263-81.

Goodwin PJ, Ennis M, Bordeleau LJ, Pritchard KI, Trudeau ME, Koo J, et al. Health-related quality of life and psychosocial status in breast cancer prognosis: analysis of multiple variables. J Clin Oncol 2004;22(20):4184-92.

Goossens ME, Rutten-van MIken MP, Vlaeyen JW, van der Linden SM. The cost diary: a method to measure direct and indirect costs in cost-effectiveness research. J Clin Epidemiol 2000;53(7):688-695.

Grol R. Personal paper. Beliefs and evidence in changing clinical practice. BMJ 1997;315(7105):418-21.

Grol R, Grimshaw J. From best evidence to best practice: effective implementation of change in patients' care. Lancet 2003;362(9391):1225-30.

Grol RP, Bosch MC, Hulscher ME, Eccles MP, Wensing M. Planning and studying improvement in patient care: the use of theoretical perspectives. Milbank Q 2007;85(1):93-138.

Grunfeld E. Optimizing follow-up after breast cancer treatment. Current opinion in obstetrics and gynaecology 2009;21(1):92-6.

Grunfeld E, Fitzpatrick R, Mant D, Yudkin P, Adewuyi-Dalton R, Stewart J, et al. Comparison of breast cancer patient satisfaction with follow-up in primary care versus specialist care: results from a randomized controlled trial. Br J Gen Pract 1999a;49(446):705-10.

Grunfeld E, Gray A, Mant D, Yudkin P, Adewuyi-Dalton R, Coyle D, et al. Follow-up of breast cancer in primary care vs specialist care: results of an economic evaluation. Br J Cancer 1999b;79(7-8):1227-33.

Grunfeld E, Levine MN, Julian JA, Coyle D, Szechtman B, Mirsky D, et al. Randomized trial of long-term follow-up for early-stage breast cancer: a comparison of family physician versus specialist care. J Clin Oncol 2006;24(6):848-55.

Grunfeld E, Mant D, Yudkin P, Adewuyi-Dalton R, Cole D, Stewart J, et al. Routine follow up of breast cancer in primary care: randomised trial. BMJ 1996;313(7058):665-9.

Gulliford T, Opomu M, Wilson E, Hanham I, Epstein R. Popularity of less frequent follow up for breast cancer in randomised study: initial findings from the hotline study. BMJ 1997;314(7075):174-7.

Guyatt GH, Osoba D, Wu AW, Wyrwich KW, Norman GR. Methods to explain the clinical significance of health status measures. Mayo Clinic proceedings 2002;77(4):371-83.

Hagedoorn M, Uijl SG, Van Sonderen E, Ranchor AV, Grol BM, Otter R, et al. Structure and reliability of Ware's Patient Satisfaction Questionnaire III: patients' satisfaction with oncological care in the Netherlands. Med Care 2003;41(2):254-63.

Harnett A, Smallwood J, Titshall V, Champion A. Diagnosis and treatment of early breast cancer, including locally advanced disease--summary of NICE guidance. BMJ 2009;338:b438. 
Helgesen F, Andersson SO, Gustafsson O, Varenhorst E, Gobén B, Carnock S, et al. Follow-up of prostate cancer patients by on-demand contacts with a specialist nurse: a randomized study. Scandinavian journal of urology and nephrology 2000;34(1):55-61.

Helgeson VS, Cohen S, Schulz R, Yasko J. Education and peer discussion group interventions and adjustment to breast cancer. Arch Gen Psychiatry 1999;56(4):340-7.

Helgeson VS, Cohen S, Schulz R, Yasko J. Group support interventions for women with breast cancer: who benefits from what? Health Psychol 2000;19(2):107-14.

Helgeson VS, Cohen S, Schulz R, Yasko J. Long-term effects of educational and peer discussion group interventions on adjustment to breast cancer. Health Psychol 2001;20(5):387-92.

Helgesson O, Lissner L, Mansson J, Bengtsson C. Quality of life in cancer survivors as observed in a population study of Swedish women. Scand J Prim Health Care 2007:1-6.

Hensher D, Rose J, Greene W. Applied Choice Analysis: A Primer. New York: Cambridge University Press; 2005.

Hodgson TA. Costs of illness in cost-effectiveness analysis. A review of the methodology. PharmacoEconomics 1994;6(6):536-552.

Houlihan NG. Transitioning to cancer survivorship: plans of care. Oncology (Williston Park) 2009;23(8 Suppl):42-8.

Husted JA, Cook RJ, Farewell VT, Gladman DD. Methods for assessing responsiveness: a critical review and recommendations. J Clin Epidemiol 2000;53(5):459-68.

Jackson JL, Chamberlin J, Kroenke K. Predictors of patient satisfaction. Soc Sci Med 2001;52(4):609-20.

Jacobs HJ, van Dijck JA, de Kleijn EM, Kiemeney LA, Verbeek AL. Routine follow-up examinations in breast cancer patients have minimal impact on life expectancy: a simulation study. Ann Oncol 2001;12(8):110713.

James ND, Guerrero D, Brada M. Who should follow up cancer patients? Nurse specialist based outpatient care and the introduction of a phone clinic system. Clin Oncol (R Coll Radiol) 1994;6(5):283-7.

Janssen MF, Birnie E, Bonsel GJ. Quantification of the level descriptors for the standard EQ-5D three-level system and a five-level version according to two methods. Qual Life Res 2008a;17(3):463-73.

Janssen MF, Birnie E, Haagsma JA, Bonsel GJ. Comparing the standard EQ-5D three-level system with a five-level version. Value Health 2008b;11(2):275-84.

Jones B, Jarvis P, Lewis JA, Ebbutt AF. Trials to assess equivalence: the importance of rigorous methods. BMJ 1996;313(7048):36-9.

Joosten EAG, DeFuentes-Merillas L, de Weert GH, Sensky T, van der Staak CPF, de Jong CAJ. Systematic Review of the Effects of Shared Decision-Making on Patient Satisfaction, Treatment Adherence and Health Status. Psychother Psychosom 2008;77:219-226 
Khatcheressian JL, Wolff AC, Smith TJ, Grunfeld E, Muss HB, Vogel VG, et al. American Society of Clinical Oncology 2006 update of the breast cancer follow-up and management guidelines in the adjuvant setting. J Clin Oncol 2006;24(31):5091-7.

Kiemeney LALM, Lemmers FAMO, Verhoeven RHA, Aben KKH, Honing C, de Nooijer J, et al. [The risk of cancer in the Netherlands]. Nederlands tijdschrift voor geneeskunde 2008;152(41):2233-2241.

Kimman ML, Bloebaum MM, Dirksen CD, Houben RM, Lambin P, Boersma $\sqcup$. Patient satisfaction with nurse-led telephone follow-up after curative treatment for breast cancer. BMC Cancer 2010a;10(1):174.

Kimman ML, Dellaert BG, Boersma L, Lambin P, Dirksen CD. Follow-up after treatment for breast cancer: one strategy fits all? An investigation of patient preferences using a discrete choice experiment. Acta Oncol 2010b;49(3):328-37.

Kimman ML, Dirksen CD, Lambin P, Boersma L. Responsiveness of the EQ-5D in breast cancer patients in their first year after treatment. Health and quality of life outcomes 2009;7(1):11.

Kimman ML, Voogd AC, Dirksen CD, Falger P, Hupperets P, Keymeulen K, et al. Follow-up after curative treatment for breast cancer: why do we still adhere to frequent outpatient clinic visits? Eur J Cancer 2007a;43(4):647-53.

Kimman ML, Voogd AC, Dirksen CD, Falger P, Hupperets P, Keymeulen K, et al. Improving the quality and efficiency of follow-up after curative treatment for breast cancer - rationale and study design of the MaCare trial. BMC Cancer 2007b;7(1):1.

Kizer KW. Patient centred care: essential but probably not sufficient. Quality \& Safety in Health Care 2002;11(2):117-118.

Kjaer T, Gyrd-Hansen D. Preference heterogeneity and choice of cardiac rehabilitation program: results from a discrete choice experiment. Health Policy 2008;85(1):124-32.

Koinberg I, Engholm GB, Genell A, Holmberg L. A health economic evaluation of follow-up after breast cancer surgery: results of an rct study. Acta Oncol 2009;48(1):99-104.

Koinberg I, Holmberg L, Fridlund B. Satisfaction with routine follow-up visits to the physician--the needs of patients with breast cancer. Acta Oncol 2001;40(4):454-9.

Koinberg I, Langius Eklof A, Holmberg L, Fridlund B. The usefulness of a multidisciplinary educational programme after breast cancer surgery: a prospective and comparative study. Eur J Oncol Nurs 2006b;10(4):273-82.

Koinberg IL, Fridlund B, Engholm GB, Holmberg L. Nurse-led follow-up on demand or by a physician after breast cancer surgery: a randomised study. Eur J Oncol Nurs 2004;8(2):109-17; discussion 118-20.

Koopmanschap MA, Rutten FF, van Ineveld BM, van Roijen L. The friction cost method for measuring indirect costs of disease. Health Econ 1995;14(2):171-89.

Krabbe PF, Peerenboom L, Langenhoff BS, Ruers TJ. Responsiveness of the generic EQ-5D summary measure compared to the disease-specific EORTC QLQ C-30. Qual Life Res 2004;13(7):1247-53.

Krahn M, Bremner KE, Tomlinson G, Ritvo P, Irvine J, Naglie G. Responsiveness of disease-specific and generic utility instruments in prostate cancer patients. Qual Life Res 2007;16(3):509-22. 
Lancaster KJ. A new approach to consumer theory. Journal of Political Economy 1966;74(2):132.

Lancsar E, Louviere J. Conducting discrete choice experiments to inform healthcare decision making: a user's guide. PharmacoEconomics 2008;26(8):661-77.

Lazarus RS, Folkman S. Stress, Appraisal and Coping. New York: Guilford; 1984.

Lewis R, Neal RD, Williams NH, France B, Wilkinson C, Hendry M, et al. Nurse-led vs. conventional physician-led follow-up for patients with cancer: systematic review. Journal of advanced nursing 2009;65(4):706-23.

Lidgren M, Wilking N, Jnsson B, Rehnberg C. Resource use and costs associated with different states of breast cancer. International journal of technology assessment in health care 2007;23(2):223-231.

Linder-Pelz S. Social psychological determinants of patient satisfaction: A test of five hypotheses. social science \& medicine 1982;16(5):583-589.

Loprinzi CL, Hayes D, Smith T. Doc, shouldn't we be getting some tests? J Clin Oncol 2000;18(11):23458.

Louviere J, Hensher D, Swait J. Stated Choice Methods: Analysis and Application: Cambridge University Press; 2000.

Manca A, Hawkins N, Sculpher M. Estimating mean QALYs in trial-based cost-effectiveness analysis: the importance of controlling for baseline utility. Health Econ 2005;14(5):487-496.

Mao JJ, Bowman MA, Stricker CT, DeMichele A, Jacobs L, Chan D, et al. Delivery of survivorship care by primary care physicians: the perspective of breast cancer patients. J Clin Oncol 2009;27(6):933-8.

Marcus AC, Garrett KM, Cella D, Wenzel L, Brady MJ, Fairclough D, et al. Can telephone counseling posttreatment improve psychosocial outcomes among early stage breast cancer survivors? Psychooncology 2009; Nov 25.

Marcus AC, Garrett KM, Cella D, Wenzel LB, Brady MJ, Crane LA, et al. Telephone counseling of breast cancer patients after treatment: a description of a randomized clinical trial. Psychooncology 1998;7(6):470-82.

Mason J, Freemantle N, Nazareth I, Eccles M, Haines A, Drummond M. When is it cost-effective to change the behavior of health professionals? JAMA 2001;286(23):2988-92.

McFadden D. Conditional logit analysis of qualitative choice behavior. In: Frontiers in Econometrics. New York: Academic Press 1974.

McLachlan SA, Devins GM, Goodwin PJ. Validation of the European Organization for Research and Treatment of Cancer Quality of Life Questionnaire (QLQ-C30) as a measure of psychosocial function in breast cancer patients. Eur J Cancer 1998;34(4):510-7.

Meneses KD, McNees P, Loerzel VW, Su X, Zhang Y, Hassey LA. Transition from treatment to survivorship: effects of a psychoeducational intervention on quality of life in breast cancer survivors. Oncology nursing forum 2007;34(5):1007-16. 
Meropol N, Schrag D, Smith T, Mulvey T, Langdon R, Blum D, et al. American Society of Clinical Oncology guidance statement: the cost of cancer care. J Clin Oncol 2009;27(23):3868-3874.

Montazeri A, Vahdaninia M, Harirchi I, Ebrahimi M, Khaleghi F, Jarvandi S. Quality of life in patients with breast cancer before and after diagnosis: an eighteen months follow-up study. BMC Cancer 2008;8(1):330.

Montgomery DA. Follow-up by telephone after treatment for breast cancer. BMJ 2009;338:a2753.

Montgomery DA, Krupa K, Cooke TG. Locoregional relapse after breast cancer: most relapses occur late and are not clinically detected. Breast J 2009;15(2):163-7.

Montgomery DA, Krupa K, Wilson C, Cooke TG. Automated telephone follow-up after breast cancer: an acceptability and feasibility pilot study. Br J Cancer 2008a;99(5):704-10.

Montgomery DA, Krupa K, Wilson C, Cooke TG. Patients' expectations for follow-up in breast cancer - A preliminary, questionnaire-based study. Breast 2008b.

Montgomery DA, Krupa K, Cooke TG. Follow-up in breast cancer: does routine clinical examination improve outcome? A systematic review of the literature. $\mathrm{Br} J$ Cancer 2007;97(12):1632-41.

Nagel GC, Schmidt S, Strauss BM, Katenkamp D. Quality of life in breast cancer patients: a cluster analytic approach. Empirically derived subgroups of the EORTC-QLQ BR 23--a clinically oriented assessment. Breast Cancer Res Treat 2001;68(1):75-87.

NCCN. NCCN practice guidelines for the management of psychosocial distress. National Comprehensive Cancer Network. Oncology (Williston Park) 1999;13(5A):113-47.

NICE. Guidance on cancer services: Improving supportive and palliative care for adults with cancer. London, United Kingdom: National Institute for Clinical Excellence; 2004.

Oostenbrink JB, Bouwmans CAM, Koopmanschap MA, Rutten FFH. Handleiding voor kostenonderzoek, methoden en standaard kostprijzen voor economische evaluaties in de gezondheidszorg. Diemen: College voor zorgverzekeringen; 2004.

Osoba D, Rodrigues G, Myles J, Zee B, Pater J. Interpreting the significance of changes in health-related quality-of-life scores. J Clin Oncol 1998;16(1):139-44.

Osoba D, Zee B, Pater J, Warr D, Kaizer L, Latreille J. Psychometric properties and responsiveness of the EORTC quality of Life Questionnaire (QLQ-C30) in patients with breast, ovarian and lung cancer. Qual Life Res 1994;3(5):353-64.

Parkin DM, Bray F, Ferlay J, Pisani P. Global cancer statistics, 2002. CA Cancer J Clin 2005;55(2):74-108.

Pearlin LI, Schooler C. The structure of coping. J Health Soc Behav 1978;19(1):2-21.

Pennery E, Mallet J. A preliminary study of patients' perceptions of routine follow-up after treatment for breast cancer. Eur J Oncol Nurs 2000;4(3):138-45; discussion 146-7.

Pestalozzi B, Castiglione M. Primary breast cancer: ESMO clinical recommendations for diagnosis, treatment and follow-up. Ann Oncol 2008;19 Suppl 2:ii7-10. 
Pestalozzi BC, Luporsi-Gely E, Jost LM, Bergh J. ESMO Minimum Clinical Recommendations for diagnosis, adjuvant treatment and follow-up of primary breast cancer. Ann Oncol 2005;16(suppl_1):i7-9.

Piaggio G, Elbourne DR, Altman DG, Pocock SJ, Evans SJ. Reporting of noninferiority and equivalence randomized trials: an extension of the CONSORT statement. JAMA 2006;295(10):1152-60.

Pickard AS, Wilke CT, Lin H-W, Lloyd A. Health utilities using the EQ-5D in studies of cancer. PharmacoEconomics 2007;25(5):365-84.

Renton JP, Twelves CJ, Yuille FA. Follow-up in women with breast cancer: the patients' perspective. Breast 2002;11(3):257-61.

Revicki D, Hays RD, Cella D, Sloan J. Recommended methods for determining responsiveness and minimally important differences for patient-reported outcomes. J Clin Epidemiol 2008;61(2):102-9.

Rojas MP, Telaro E, Russo A, Moschetti I, Coe L, Fossati R, et al. Follow-up strategies for women treated for early breast cancer. Cochrane Database Syst Rev 2005(1):CD001768.

Roland M, Torgerson DJ. What are pragmatic trials? BMJ 1998;316(7127):285.

Rosselli Del Turco M, Palli D, Cariddi A, Ciatto S, Pacini P, Distante V. Intensive diagnostic follow-up after treatment of primary breast cancer. A randomized trial. National Research Council Project on Breast Cancer follow-up. JAMA 1994;271(20):1593-7.

Rutgers EJ, van Slooten EA, Kluck HM. Follow-up after treatment of primary breast cancer. Br J Surg 1989;76(2):187-90.

RVZ. Sensible and sustainable Care. Zoetermeer, The Netherlands: Raad voor de Volksgezondheid en Zorg; 2006.

Ryan M. A role for conjoint analysis in technology assessment in health care? International journal of technology assessment in health care 1999;15(3):443-57.

Ryan M, Gerard K. Using discrete choice experiments to value health care programmes: current practice and future research reflections. Applied health economics and health policy 2003;2(1):55-64.

Salkeld G, Ryan M, Short L. The veil of experience: do consumers prefer what they know best? Health Econ 2000;9(3):267-70.

Sandgren AK, McCaul KD. Short-term effects of telephone therapy for breast cancer patients. Health Psychol 2003;22(3):310-5.

Sardell S, Sharpe G, Ashley S, Guerrero D, Brada M. Evaluation of a nurse-led telephone clinic in the follow-up of patients with malignant glioma. Clin Oncol (R Coll Radiol) 2000;12(1):36-41.

Schafer JL. Multiple imputation: a primer. Statistical methods in medical research 1999;8(1):3-15.

Schapira DV. Breast cancer surveillance - a cost-effective strategy. Breast Cancer Res Treat 1993;25(2):107-11. 
Schou I, Ekeberg O, Sandvik L, Hjermstad MJ, Ruland CM. Multiple predictors of health-related quality of life in early stage breast cancer. Data from a year follow-up study compared with the general population. Qual Life Res 2005;14(8):1813-23.

Sculpher MJ, Claxton K, Drummond M, McCabe C. Whither trial-based economic evaluation for health care decision making? Health Econ 2006;15(7):677-87.

Sculpher MJ, Pang FS, Manca A, Drummond MF, Golder S, Urdahl H, et al. Generalisability in economic evaluation studies in healthcare: a review and case studies. Health Technol Assess 2004;8(49):iii-iv, 1-192.

Sitzia J. How valid and reliable are patient satisfaction data? An analysis of 195 studies. International Journal for Quality in Health Care 1999;11(4):319-328.

Sitzia J, Wood N. Patient satisfaction: a review of issues and concepts. Social Science \& Medicine 1997;45(12):1829-1843.

Sterne JA, White IR, Carlin JB, Spratt M, Royston P, Kenward MG, et al. Multiple imputation for missing data in epidemiological and clinical research: potential and pitfalls. BMJ 2009;338:b2393.

Street DJ, Burgess L, Louviere JJ. Quick and easy choice sets: Constructing optimal and nearly optimal stated choice experiments. International Journal of Research in Marketing 2005;22(4):459-470.

Struikmans H, Nortier JW, Rutgers EJ, Zonderland HM, Bontenbal M, Elkhuizen PH, et al. [Guideline 'Treatment of breast cancer 2008' (revision)]. Ned Tijdschr Geneeskd 2008;152(46):2507-11.

Taves DR. Minimization: a new method of assigning patients to treatment and control groups. Clin Pharmacol Ther 1974;15(5):443-53.

te Boekhorst DS, Peer NG, van der Sluis RF, Wobbes T, Ruers TJ. Periodic follow-up after breast cancer and the effect on survival. Eur J Surg 2001;167(7):490-6.

Terwee CB, Dekker FW, Wiersinga WM, Prummel MF, Bossuyt PM. On assessing responsiveness of healthrelated quality of life instruments: guidelines for instrument evaluation. Qual Life Res 2003;12(4):34962.

TOMBOLA. Options for managing low grade cervical abnormalities detected at screening: cost effectiveness study. BMJ 2009;339:b2549.

Train K. Discrete Choice Methods with Simulation: Cambridge University Press, U.K.; 2003.

van Asselt AD, van Mastrigt GA, Dirksen CD, Arntz A, Severens JL, Kessels AG. How to deal with cost differences at baseline. PharmacoEconomics 2009;27(6):519-28.

van de Willige G, Wiersma D, Nienhuis FJ, Jenner JA. Changes in quality of life in chronic psychiatric patients: a comparison between EuroQol (EQ-5D) and WHOQoL. Qual Life Res 2005;14(2):441-51.

van der Ploeg HM. Validatie van de Zelf-Beoordelings Vragenlijst (een Nederlandstalige bewerking van de Spielberger State-Trait Anxiety Inventory). Nederlands Tijdschrift voor de Psychologie 1980;35:243-9. 
Waldmann A, Pritzkuleit R, Raspe H, Katalinic A. The OVIS study: health related quality of life measured by the EORTC QLQ-C30 and -BR23 in German female patients with breast cancer from Schleswig-Holstein. Qual Life Res 2007;16(5):767-76.

Ware JE, Jr. Effects of acquiescent response set on patient satisfaction ratings. Med Care 1978;16(4): 327-36.

Ware JE, Jr., Sherbourne CD. The MOS 36-item short-form health survey (SF-36). I. Conceptual framework and item selection. Med Care 1992;30(6):473-83.

Ware JE, Snyder MK, Wright WR, Davies AR. Defining and measuring patient satisfaction with medical care. Evaluation and Program Planning 1983;6(3-4):247-263.

Weinstein MC, Stason WB. Foundations of cost-effectiveness analysis for health and medical practices. N Engl J Med 1977;296(13):716-21.

Wheeler T, Stenning S, Negus S, Picken S, Metcalfe S. Evidence to support a change in follow-up policy for patients with breast cancer: time to first relapse and hazard rate analysis. Clin Oncol (R Coll Radiol) 1999;11(3):169-73.

Wiebe S, Guyatt G, Weaver B, Matijevic S, Sidwell C. Comparative responsiveness of generic and specific quality-of-life instruments. J Clin Epidemiol 2003;56(1):52-60.

Williams B. Patient satisfaction: a valid concept? Soc Sci Med 1994;38(4):509-16.

Worthington K. Customer satisfaction in the emergency department. Emergency medicine clinics of North America 2004;22(1):87-102.

Wyatt G, Kurtz ME, Liken M. Breast cancer survivors: an exploration of quality of life issues. Cancer Nurs 1993;16(6):440-8. 


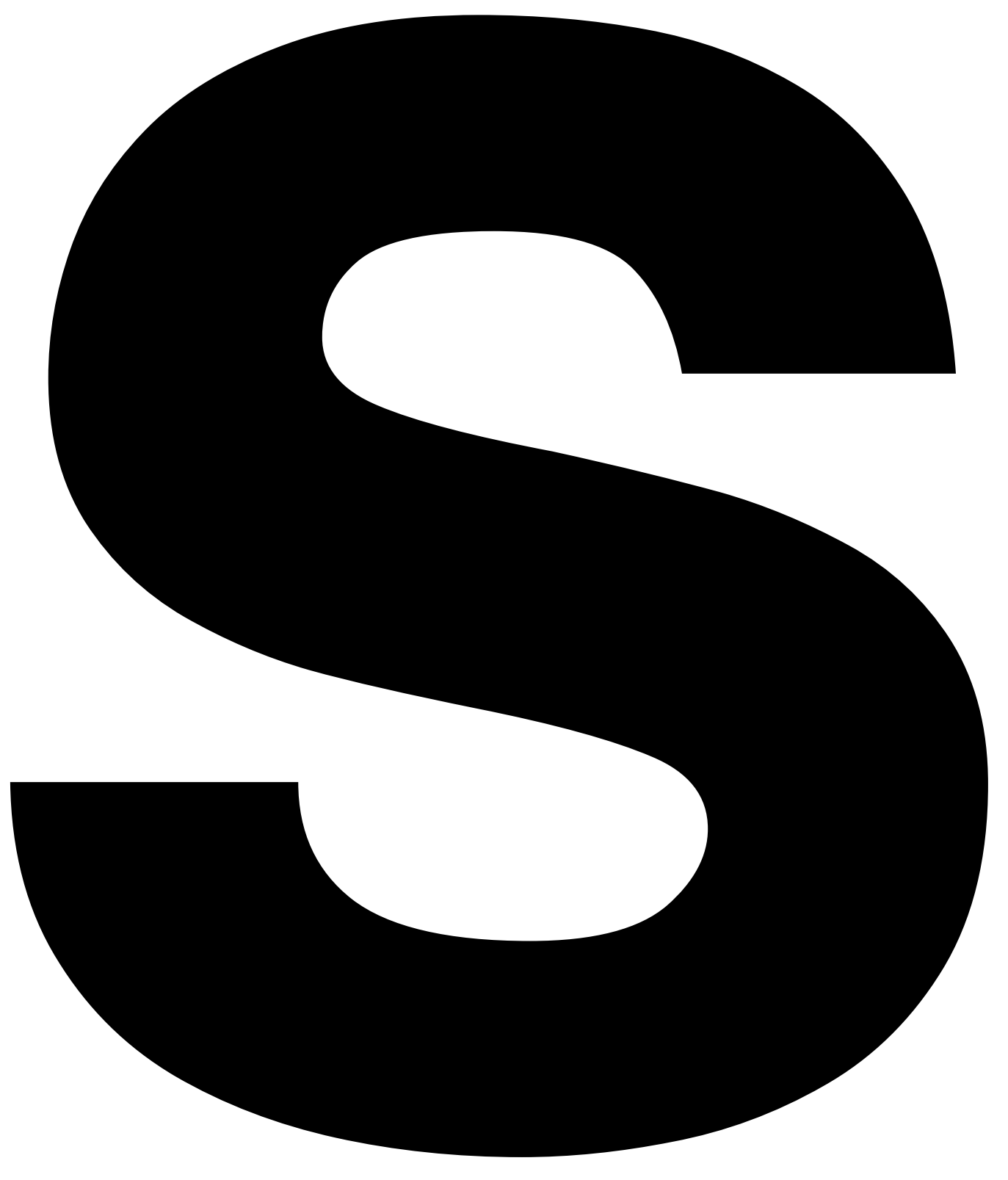




\section{Summary}

There is much debate regarding the value of routine follow-up of women with breast cancer. Current follow-up patterns depend heavily on expensive and scarce specialized knowledge for routine history taking and for physical examinations, but do not always provide optimal care for patients. Hence, there is a need to evaluate costs in relation to benefits of current and alternative follow-up strategies. This thesis is based on five years of research concerning breast cancer follow-up, focused on the first year after completion of treatment.

CHAPTER 1 provides background information on the follow-up of women with breast cancer, economic evaluations in health care and discrete choice experimentation. The chapter concludes with the specific objectives and outline of the thesis.

CHAPTER 2 provides an overview on current literature on breast cancer follow-up; guidelines for follow-up, effectiveness of follow-up, the needs of patients and proposed alternative strategies. The optimum frequency or duration of follow-up visits has not yet been properly established and there remains wide discrepancy in guideline statements of the frequency of visits. Several studies show that neither the frequency nor intensity of follow-up improves the chances of cure. Furthermore, instead of providing psychosocial 
support, outpatient clinic visits may induce anxiety because of the potential of detecting tumor relapse. Hence, not only do the current frequent follow-up strategies seem to miss their most important goals, they also depend heavily on expensive and scarce specialized knowledge for routine history taking and physical examinations. As a result, alternative follow-up strategies have been proposed, such as follow-up by the general practitioner, and nurse-led follow-up or telephone follow-up. Although studies demonstrate that these alternative follow-up strategies can be equally effective as traditional hospital followup, as measured by a variety of outcomes, these strategies are not yet widely applied in clinical practice. Results may not have been sufficiently convincing, or perhaps medical specialists believe that patients need frequent reassurance. Moreover, patients may have false expectations that frequent follow-up will result in better overall survival. Since brief interventions based on self-education and information are able to improve quality of life, we hypothesize that these interventions may also lead to a better acceptance of reduced follow-up by both patients and professionals. For this reason, a randomized controlled trial (RCT) was developed to investigate the effectiveness and cost-effectiveness of a reduced follow-up strategy (i.e. nurse-led telephone follow-up), and of a short educational group program (EGP) in addition to the follow-up.

CHAPTER 3 provides a detailed description of the design and execution of the RCT, the socalled MaCare trial. Participants $(n=320)$ were randomly assigned to one of four follow-up strategies (study arms) during the first 18 months after treatment; i.e. 1) hospital followup every three months, including mammography at 12 months; 2) nurse-led telephone follow-up every three months, plus hospital visit and mammography at 12 months; 3) arm 1 plus educational group program (EGP); 4) arm 2 plus EGP.

For patients randomized to hospital follow-up (arms 1 and 3), follow-up visits consisted of physical examination and medical history taking by a medical specialist or breast care nurse (BCN). Nurse-led telephone follow-up (arms 2 and 4) was done by open discussion and a semi-structured questionnaire including screening for physical -especially loco regional- and psychosocial symptoms, treatment side effects, compliance with hormonal therapy plus an open discussion of these issues. In study arms 3 and 4, patients and their partners were invited to participate in the EGP. The EGP consisted of two group-sessions, led by a breast care nurse and health psychologist, in which physical and psychosocial sequelae of diagnosis and treatment were discussed. The primary endpoint of the trial was health-related quality of life (HRQoL) at 12 months after treatment, measured by the EORTC QLQ-C30. Secondary outcomes were perceived feelings of control, anxiety, patient satisfaction and costs. An economic evaluation was performed from a societal perspective, comparing costs and quality-adjusted life years (QALYs) of the four strategies. All data was collected at baseline and 3, 6, 12 and 18 months after treatment.

In CHAPTER 4 the results of the RCT are reported regarding the effectiveness of both nurse-led telephone follow-up and the EGP in addition to follow-up. Results showed that 
most hospital clinic visits in the first year after treatment could be replaced by nurse-led telephone follow-up without loss of HRQoL or increase in feelings of anxiety. Additionally, patients with nurse-led telephone follow-up showed the same levels of role and emotional functioning, and feelings of control as patients with hospital follow-up by 12 months. Adding the EGP to follow-up may be appropriate to address information needs, but it did not lead to a significant improvement in HRQoL, emotional functioning or feelings of control as compared to follow-up without an EGP. It was concluded that nurse-led telephone followup may be appropriate way to reduce clinic visits and represents an accepted alternative strategy, while an EGP does not unequivocally affect positive HRQoL outcomes.

The EQ-5D is a generic HRQoL measure that is recommended for the use in economic evaluations of health interventions. Concerns have been raised about its ability to pick up meaningful changes in health in cancer populations. In CHAPTER $\mathbf{5}$ the results of a study investigating the responsiveness of the EQ-5D in breast cancer patients in their first year after treatment are reported. The validated HRQoL measure EORTC QLQ-C30 was used as a reference instrument to determine meaningful changes in health and identify subgroups of patients. Responsiveness was evaluated by calculating standardized response means (SRMs) and performing analysis of variance procedures. The two HRQoL measures were completed two weeks and one year after finalizing curative treatment for breast cancer ( $n=192)$. SRMs of the EQ-5D utility score were close to zero in the subgroup reporting no change and increased and decreased adequately in the subgroups reporting small and moderate changes. Additional analysis of variance procedures showed that the EQ-5D was able to differentiate between subgroups of patients with no change and moderate-large deterioration or improvement in health. Importantly, the EQ-5D appeared an appropriate measure for the purpose of economic evaluations of health intervention in breast cancer patients after treatment.

CHAPTER 6 reports on the economic evaluation, performed alongside the RCT, to determine the most cost-effective follow-up strategy. The economic evaluation $(n=299)$ compared one-year costs and effects of the four follow-up strategies as described in chapter 3. Costs were measured using patient level data from cost diaries and hospital registrations. Quality-adjusted life years (QALYs) were measured using the EQ-5D. Outcomes were expressed in incremental cost-effectiveness ratios and cost-effectiveness acceptability curves. Results showed that hospital follow-up plus EGP yielded most QALYs (0.776), but also incurred the highest mean annual costs (€4914). The incremental costeffectiveness ratio (ICER) of hospital follow-up plus EGP versus the next best alternative telephone follow-up plus EGP (0.772 QALYs and €3971) amounted to €235.750/QALY. As hospital and telephone follow-up without EGP both had higher costs and less QALYs than telephone follow-up plus EGP, the former were judged inferior. Assuming a threshold value of $€ 40.000$ per QALY, telephone follow-up plus EGP was the preferred strategy. The probability of this strategy being most cost-effective ranged from $49 \%$ to $62 \%$ for different 
QALY threshold values. Secondary and sensitivity analyses showed that these results were robust. Thus, nurse-led telephone follow-up plus EGP instead of traditional, routine hospital clinic visits, during the first year after breast cancer treatment, may lead to a substantial cost reduction.

Although the economic evaluation showed that telephone follow-up plus EGP offers good value for money, a policy maker may wish to incorporate other factors such as patient satisfaction and preferences. CHAPTER 7 concerns patients' satisfaction with nurse-led telephone follow-up compared to traditional hospital follow-up for the first 12 months after treatment. Data on patient satisfaction were collected at baseline, and 3, 6 and 12 months after treatment, using the Dutch version of Ware's Patient Satisfaction Questionnaire III (PSQ III). In addition to general satisfaction, the PSQ III reports on satisfaction scores for technical competence, interpersonal aspects and access of care. It was found that there were no meaningful differences in satisfaction scores between patients who were randomized to nurse-led telephone follow-up and those randomized to hospital follow-up.

In CHAPTER 8 patient preferences for characteristics of breast cancer follow-up are explored. A discrete choice experiment survey with 16 choice tasks was filled out by 331 breast cancer patients who were on average 14 months after end of treatment. Each choice task consisted of two hypothetical follow-up scenarios for the first year after treatment, described by levels of the following characteristics; attendance at an educational group program (EGP), frequency of visits, waiting time, contact mode and type of health care provider. Results showed that the health care provider and contact mode were the most important characteristics of follow-up to respondents. The medical specialist was the most preferred to perform the follow-up, but a combination of the medical specialist and breast care nurse alternating was also acceptable. Face-to-face contact was strongly preferred to telephone contact. Heterogeneity in preference between respondents was strong, especially for the health care provider ( $28 \%$ would positively value a change from medical specialist to nurse-led follow-up) and attendance at the EGP ( $47 \%$ of respondents preferred participation to no participation). Follow-up by a medical specialist was valued more positively by older (>60 years) than younger respondents. Furthermore, older respondents and those with a low level of education were less likely to choose a scenario that included attending the EGP. Importantly, respondents with previous experience with the EGP and nurse-led follow-up had a significantly stronger preference for these characteristics. Treatment modality did not influence preferences. Heterogeneity in preferences for most attributes suggested that one follow-up strategy may not fit all.

CHAPTER 9 discusses the main research findings of this thesis, the strength of the studies performed, as well as some methodological considerations regarding the research. The RCT was performed as a pragmatic trial and the economic evaluation closely reflected effectiveness and resource use that would be observed in real practice. Since QALYs fail to 
incorporate non-health related issues in the decision making process, such as satisfaction with and preferences for process outcomes of follow-up (e.g. accessibility, provision of information and continuity), these aspects were measured separately. However, preferences and satisfaction were difficult concepts to measure and concerns regarding the validity of the findings and the influence of chosen study samples were discussed. Moreover, merging evidence from our economic evaluation and patients' perspectives created some conflict, since follow-up based on individual preferences was likely to differ from the follow-up recommended in terms of cost-effectiveness. It was recommended to change current breast cancer guidelines from hospital follow-up to nurse-led telephone follow-up plus EGP. Future research may investigate the feasibility and cost-effectiveness of more individualized follow-up. 


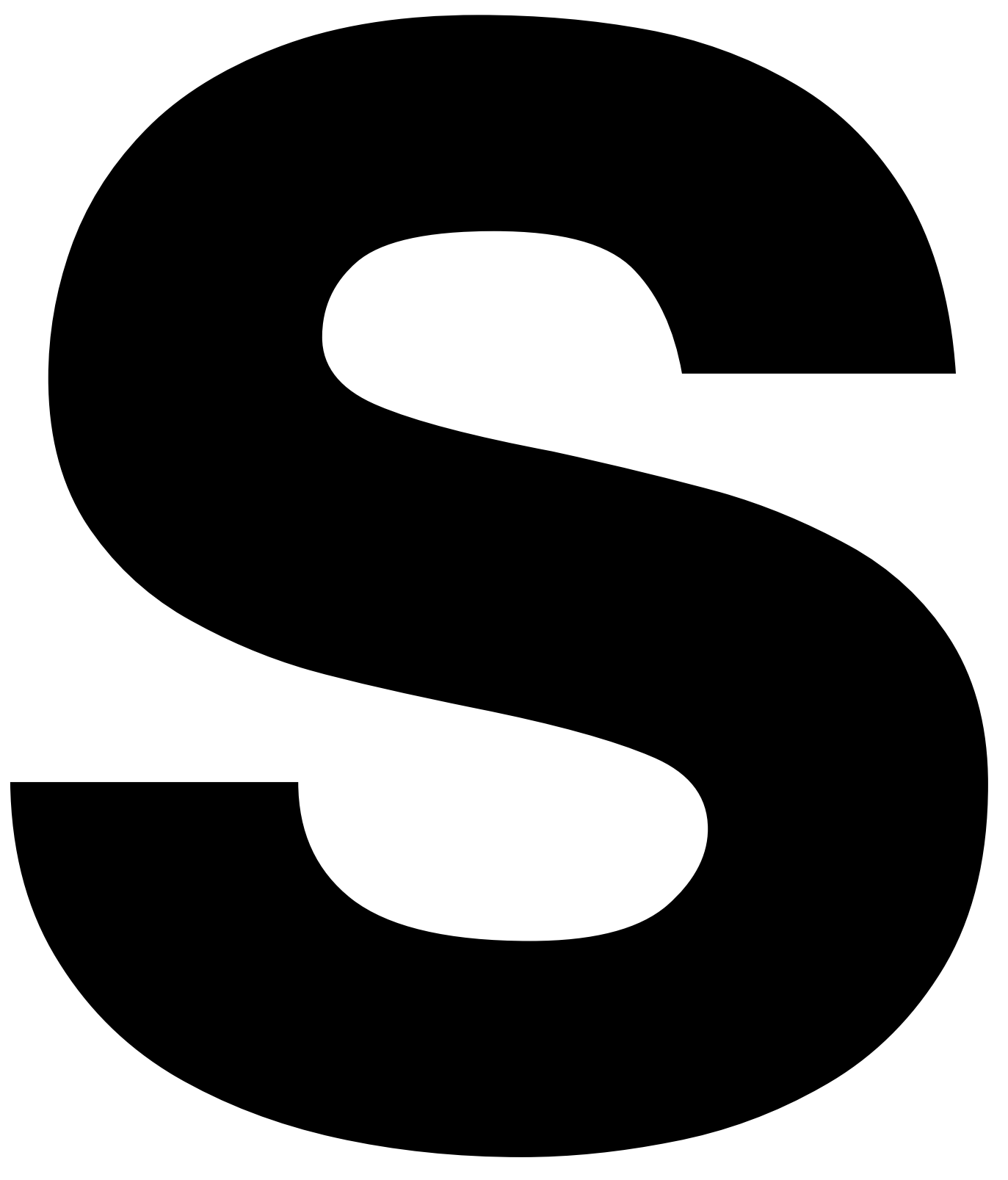




\section{Samenvatting}

Er bestaat twijfel of het frequent controleren van patiënten na de behandeling van borstkanker efficiënt is. Het huidige followup beleid leidt tot een grote belasting van de medisch specialisten en daarmee tot hoge kosten, en bovendien is de aangeboden zorg niet optimaal. Er bestaat een behoefte om de kosten en de effectiviteit van de huidige follow-up, evenals van alternatieve modellen van follow-up, te onderzoeken. Dit proefschrift is gebaseerd op vijf jaar onderzoek naar de follow-up na borstkanker met de focus op het eerste jaar na de behandeling.

HOOFDSTUK 1 geeft achtergrond informatie over de huidige follow-up na borstkanker, economische evaluaties in de zorg en keuze-experimenten. Het hoofdstuk wordt afgesloten met de specifieke doelstellingen en inhoud van het proefschrift.

In HOOFDSTUK 2 wordt een overzicht van de huidige literatuur over borstkanker followup gegeven; richtlijnen, effectiviteit, het patiëntenperspectief en alternatieve follow-up modellen. De optimale frequentie van follow-up controles, evenals hoe lang de followup zou moeten duren, staat niet vast en internationale richtlijnen komen niet overeen. Verschillende studies hebben laten zien dat jaarlijkse follow-up met mammografie even effectief is wat betreft de overleving als meer intensieve follow-up. Daarnaast is gebleken 
dat het bieden van psychosociale ondersteuning nog onvoldoende is in de huidige follow-up, en ook dat frequente controles angstgevoelens opwekken rondom het kunnen terugkeren van de kanker. Het huidige follow-up beleid voldoet enerzijds niet aan de voorafgestelde doelen, en anderzijds leidt het tot een grote belasting van de medisch specialisten en daarmee tot hoge kosten. Als gevolg hiervan zijn alternatieve modellen van follow-up in diverse studies onderzocht, zoals follow-up door de huisarts, de verpleegkundige en telefonische follow-up. Hoewel deze alternatieve vormen minstens zo effectief en veilig waren als traditionele follow-up in het ziekenhuis, worden deze follow-up strategieën nog niet standaard toegepast in de klinische praktijk. Dit zou kunnen komen door financiële overwegingen, maar een wellicht logischer verklaring is dat veel medisch specialisten, mogelijk onterecht, denken dat patiënten continue bevestiging en geruststelling nodig hebben. Daarnaast heeft een groot deel van de patiënten irreële verwachtingen van follow-up, namelijk dat intensieve en frequente follow-up tot een betere overleving zouden leiden. Ook economische evaluaties om de kosteneffectiviteit van alternatieve follow-up strategieën te bepalen, worden nog te weinig meegenomen in nieuwe studies. Op grond van de literatuur is het de verwachting dat verbetering in de psychosociale ondersteuning en educatie van patiënten kan leiden tot een betere acceptatie door zowel patiënten als medisch specialisten van minder frequente follow-up. Daarom werd een gerandomiseerde studie gestart, gericht op de kosten en de effectiviteit (kwaliteit van leven) van een gereduceerde follow-up strategie (namelijk telefonische follow-up door de mammacare verpleegkundige) en een educatief groepsprogramma (EGP), voor curatief behandelde borstkankerpatiënten.

HOOFDSTUK 3 geeft een gedetailleerde beschrijving van de opzet en uitvoering van de gerandomiseerde studie. Deelnemers $(n=320)$ werden door loting toegewezen aan een van vier follow-up strategieën (studie-armen) voor de eerste 18 maanden na de behandeling; 1) standaard follow-up (bezoek ziekenhuis op 3, 6, 9, 12 en 18 maanden en een jaarlijkse mammografie; 2) follow-up $1 \times$ per jaar inclusief mammografie, gecombineerd met telefonische follow-up door een mammacare verpleegkundige op dezelfde momenten als de standaard follow-up; 3) arm 1 met daarbij een groepsinterventie gericht op voorlichting (educatief groepsprogramma (EGP)); 4) arm 2 met EGP.

Voor patiënten in de ziekenhuis follow-up (arm 1 en 3) bestond de follow-up uit anamnese en lichamelijk onderzoek door een medisch specialist of mammacare verpleegkundige. De telefonische follow-up (arm 2 en 4) vond plaats in de vorm van een open gesprek waarin gescreend werd voor een aantal belangrijke symptomen van een recidief. Daarnaast informeerde de verpleegkundige naar het algemeen welbevinden van de patiënt, haar gezinsleven en werkhervatting.

In arm 3 en 4 werden patiënten (en partners) uitgenodigd deel te nemen aan het EGP. Het EGP bestond uit twee sessies van ongeveer 2,5 uur en werd geleid door een gezondheidszorg psycholoog en mammacare verpleegkundige. Er werd informatie gegeven over mogelijke late bijwerkingen van de verschillende behandelingen en hormonale 
therapie, symptomen van een eventueel recidief, evenals de mogelijke psychologische gevolgen van de diagnose en behandeling van borstkanker en hoe daar mee om te gaan. Het primaire eindpunt van de studie was gezondheidsgerelateerde kwaliteit van leven op 12 maanden na behandeling, gemeten met de EORTC QLQ-C30 vragenlijst. Secundaire uitkomstmaten waren gevoelens van controle, angst, tevredenheid en kosten. Daarnaast werd een economische evaluatie vanuit een maatschappelijk perspectief uitgevoerd, waarin kosten en QALYs (voor kwaliteit gecorrigeerde levensjaren) van de vier followup strategieën werden vergeleken. Alle gegevens werden verzameld op 3, 6, 12 en 18 maanden na randomisatie.

In HOOFDSTUK 4 worden de resultaten van de gerandomiseerde studie beschreven wat betreft de effectiviteit van zowel de telefonische follow-up door de mammacare verpleegkundige als het EGP. De meeste ziekenhuis follow-up bezoeken in het eerste jaar na de behandeling konden vervangen worden door telefonische follow-up zonder een afname van kwaliteit van leven of een toename in angstgevoelens. Telefonische followup had ook geen negatieve invloed op emotioneel functioneren of controle gevoelens. Het toevoegen van een EGP aan de follow-up leidde niet tot significante verbetering in kwaliteit van leven of andere psychologische uitkomstmaten. Er werd geconcludeerd dat telefonische follow-up een effectieve en geaccepteerde follow-up strategie is, maar dat het EGP niet tot een betere kwaliteit van leven leidt.

De EQ-5D is een generieke gezondheidsgerelateerde kwaliteit van leven vragenlijst die gebruikt wordt in economische evaluaties van gezondheidsinterventies. $\mathrm{Er}$ is echter twijfel of de EQ-5D belangrijke verschillen in gezondheid kan identificeren. In HOOFDSTUK 5 worden de resultaten beschreven van een studie die de responsiviteit van de EQ-5D onderzocht in borstkankerpatiënten in het eerste jaar na behandeling. De subschaal 'algemene gezondheid' van de EORTC QLQ-C30 werd gebruikt als een referentiemaat om veranderingen in gezondheidstoestand te bepalen en subgroepen te identificeren. Responsiviteit werd vervolgens geëvalueerd door het berekenen van de zgn. standardized response means (SRMs) en variantie analyse. De EQ-5D en EORTC QLQ-C30 waren ingevuld op 2 weken en 1 jaar na einde van de behandeling $(n=192)$. SRMs van de EQ-5D utiliteits scores waren nagenoeg gelijk aan 0 in de subgroep die geen gezondheidsverandering liet zien volgens de referentiemaat, en de SRMs namen gelijkmatig toe en af in de subgroepen die respectievelijk een verbetering of verslechtering in gezondheidstoestand lieten zien. Variantie analyse liet zien dat de EQ-5D in staat was om te differentiëren tussen subgroepen zonder gezondheidsverandering en subgroepen met een gemiddelde tot grote gezondheidsverandering. De EQ-5D lijkt een bruikbaar instrument voor economische evaluaties van gezondheidsinterventies voor patiënten na de behandeling van borstkanker. 
HOOFDSTUK 6 beschrijft de economische evaluatie die uitgevoerd was binnen de gerandomiseerde studie om de meest kosteneffectieve follow-up strategie te bepalen. De economische evaluatie $(n=299)$ vergeleek de kosten en effecten van de vier followup strategieën voor het eerste jaar na de behandeling, zoals beschreven in hoofdstuk 3. Zorggebruik was gemeten met behulp van kostendagboeken ingevuld door patiënten en de registratiesystemen van de ziekenhuizen. QALYs waren gemeten met de EQ-5D. Uitkomsten werden uitgedrukt in incrementele kosten-effectiviteitsratio's (ICER) en kosteneffectiviteit-acceptatie grafieken. Resultaten lieten zien dat ziekenhuis follow-up met het EGP resulteerde in de meeste QALYs (0.776), maar ook de hoogste kosten met zich mee bracht (€4914). De ICER van ziekenhuis follow-up met EGP versus het volgend beste alternatief (telefonische follow-up met EGP) (0.772 QALYs en €3971) was €235.750/ QALY. Ziekenhuis en telefonische follow-up zonder het EGP hadden beide hogere kosten en minder QALYs dan telefonische follow-up met EGP en werden als inferieure strategieën beschouwd. Uitgaande van een grenswaarde van $€ 40.000$ voor een QALY, werd ziekenhuis follow-up met EGP niet kosteneffectief beschouwd en was telefonische follow-up met EGP de geprefereerde strategie. De kans dat deze strategie het meest kosteneffectief was varieerde tussen de $49 \%$ en $62 \%$ voor verschillende grenswaarden van een QALY. Secundaire en gevoeligheidsanalyses lieten zien dat de resultaten robuust waren. Telefonische follow-up met EGP, in plaats van de traditionele ziekenhuis follow-up, kan tot significante kostenbesparingen in Nederland leiden.

Ondanks dat de economische evaluatie liet zien dat telefonische follow-up met EGP kosteneffectief was, kan het zijn dat beleidsmakers en/of verzekeringsmaatschappijen ook andere factoren zoals patiënten tevredenheid en voorkeuren, mee willen laten wegen in hun beleidsbeslissing. HOOFDSTUK 7 beschrijft de tevredenheid van patiënten met telefonische follow-up vergeleken met de tevredenheid van patiënten met ziekenhuis follow-up, zoals gemeten in de RCT beschreven in hoofdstuk 3. Tevredenheidsscores werden verzameld op 3, 6, 9 en 12 maanden na de behandeling met de Nederlandse versie van Ware's Patient Tevredenheidsvragenlijst, de PSQ III. Naast algemene tevredenheid meet de PSQ III ook tevredenheid wat betreft technische competentie van zorgverleners, interpersoonlijke aspecten en toegang tot de zorg. Er werden geen betekenisvolle verschillen in patiënten tevredenheid gevonden tussen telefonische followup en follow-up in het ziekenhuis.

In HOOFDSTUK 8 wordt een keuze-experiment naar voorkeuren van patiënten voor de follow-up beschreven. Het keuze-experiment bestond uit een vragenlijst met 16 keuzesets en was ingevuld door 331 borstkanker patiënten. Elke keuzeset bestond uit twee hypothetische follow-up scenario's voor het eerste jaar na de behandeling, waarin de volgende kenmerken van de follow-up beschreven stonden: deelname aan het EGP, frequentie van follow-up bezoeken, wachttijd, wijze van contact en het type zorgverlener. De resultaten lieten zien dat voor patiënten het type zorgverlener en wijze van contact 
de belangrijkste kenmerken van follow-up waren. De medisch specialist was de meest geprefereerde zorgverlener voor de follow-up, maar een combinatie van (afwisselend) de medisch specialist en mammacare verpleegkundige was ook acceptabel. Er was een sterke voorkeur voor persoonlijk contact boven telefonisch contact. Preferenties waren duidelijk heterogeen, met name voor de zorgverlener (28\% van participanten zou een verandering van medische specialist naar mammacare verpleegkundige als positief ervaren) en deelname aan het EGP (47\% van participanten had een voorkeur voor deelname t.o.v. geen deelname). Een hogere leeftijd was geassocieerd met een voorkeur voor de medisch specialist. Daarnaast bleken participanten met een hogere leeftijd en een lager opleidingsniveau een minder sterke voorkeur te hebben voor deelname aan het EGP. Ervaring met bepaalde aspecten van follow-up (zoals de mammacare verpleegkundige en het EGP) resulteerde in significant sterkere voorkeuren voor deze aspecten. Behandelingsmodaliteit had geen invloed op de preferenties van patiënten. De sterke heterogeniteit in preferenties suggereert dat eenzelfde follow-up strategie voor iedereen niet de voorkeur heeft.

In HOOFDSTUK 9 worden de hoofdbevindingen van het proefschrift samengevat en enkele methodologische vraagstukken gerelateerd aan het verrichte onderzoek bediscussieerd. De gerandomiseerde studie was pragmatisch van aard en vertegenwoordigde daarmee nauw de effectiviteit en kosteneffectiviteit in de praktijk. Aangezien de QALY enkele nietgezondheidsgerelateerde aspecten die van belang zijn in medische besliskunde negeert, zoals patiënten tevredenheid en voorkeuren voor logistieke aspecten van follow-up, werden deze aspecten apart onderzocht in dit proefschrift. Echter, patiënten tevredenheid en voorkeuren zijn moeilijk te meten en de validiteit van de gevonden resultaten werd in dit laatste hoofdstuk onder de loep genomen. Daarnaast werd ingegaan op de invloed van de resultaten van de economische evaluatie en het preferentie onderzoek op de klinische praktijk en beleid. Er werd aanbevolen de huidige richtlijn voor follow-up te veranderen van traditioneel ziekenhuis follow-up naar telefonische follow-up in combinatie met het EGP. Toekomstig onderzoek zou de haalbaarheid en kosteneffectiviteit van meer geïndividualiseerde follow-up moeten onderzoeken. 


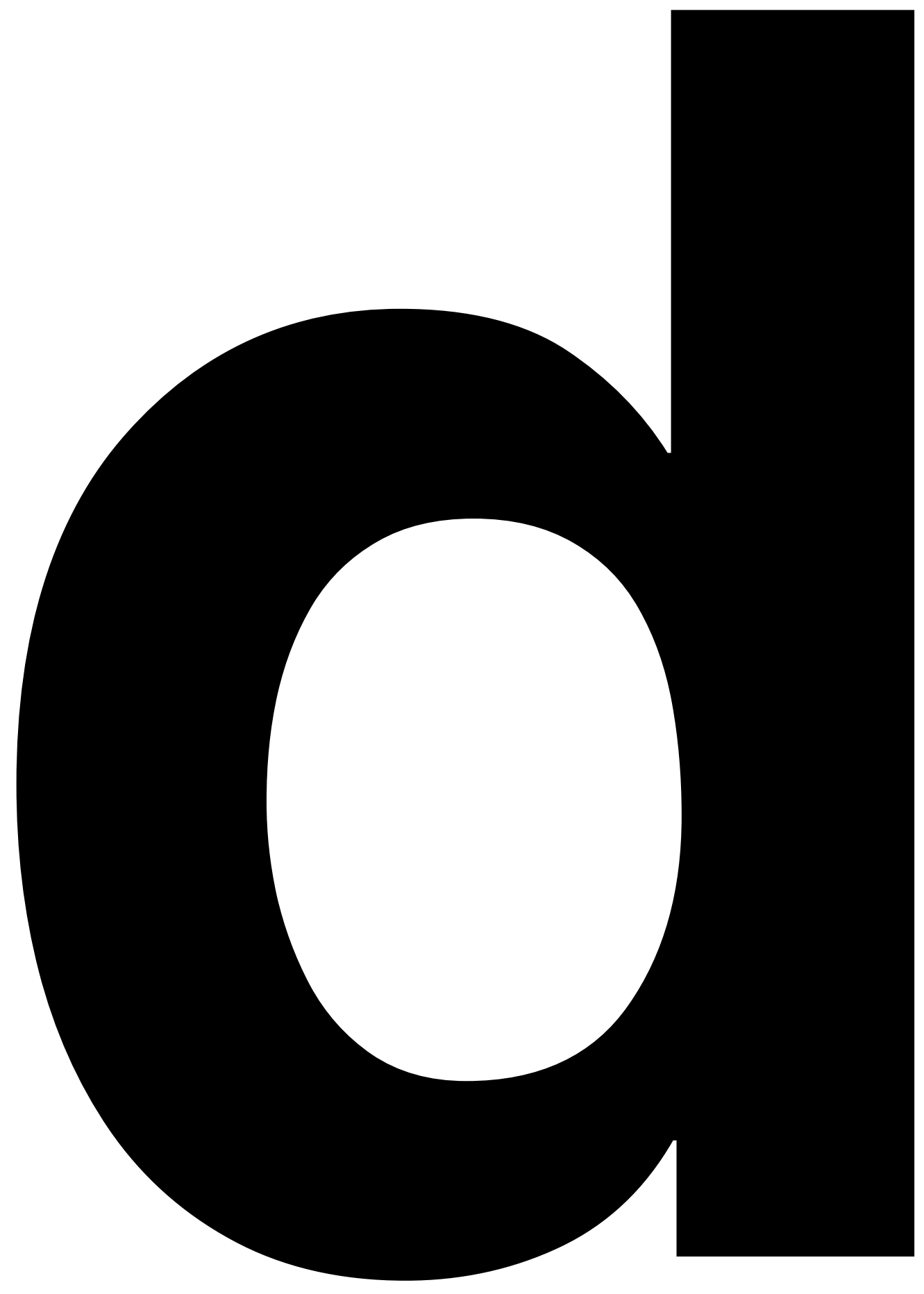


Dank!

\section{GRATEFULLY ACKNOWLEDGE}

LIESBETH BOERSMA | CARMEN DIRKSEN | PHILIPPE LAMBIN | KARIN DE BIE | ADRI VOOGD | PAUL FALGER | MARK THURING | CHRISTEL HAEKENS | CARY OBERIJE | BRIGITTE GIJSEN I PIERRE HUPPERETS I MARLENE HEBLY I CHRISTEL HAEKENS I CONNIE STARREN-GOESSENS I ELLY DE JONG I IRIS JASPERS I ANNE-MARTH CORSTJENS-LENSSEN | MAUD SCHURGERS | MONIQUE VONCKEN | ANS WILLEMS I INGRID SNIJDERS | MIEKE LAENEN | WILMA ABEN | SANDRA JANSSEN | MARJA PORTZ | DESIREE SCHREURS | MARJAN JANSSEN-WESSELS | SASKIA CLAASSEN I MARIËLLE CASTELIJNS | ANGELIQUE SMULDERS | ASTRID HOUKES | MARIKA DE WIT I DUNJA VAN DER MEER I RIET KLAASEN I HANNEKE KNIBBELER | MONIQUE DUSEE | LUC SCHEIJMANS | STEFAN BRENNINKMEIJER | HANS NUYTINCK | KRISTIEN KEYMEULEN I MAARTEN VON MEYENFELDT I JOOST VERKEYN I HANS VAN DER BIJL I YVONNE VAN RIET I MAURICE VAN DER SANGEN | FRED VAN DER ENT | FONS KESSELS | MONIQUE BLOEBAUM | RUUD HOUBEN | BENEDICT DELLAERT | JANNEKE GRUTTERS | BEOZ | KEMTA | VRIJWILLIGERS BORSTKANKERVERENIGING | ZONMW | MEMIC | INTEGRAAL KANKERCENTRUM LIMBURG | AUKJE VAN GESTEL | RENÉ VOGELS STICHTING I MAASTRO CLINIC I GROW RESEARCH INSTITUTE I TOON HERMANSHUIS MAASTRICHT I TOON HERMANSHUIS HEERLEN I INLOOPHUIS DE HONINGRAAD | DEELNEMERS MAZORG-STUDIE | DATAMANAGERS | DR. BERNARD VERBEETEN INSTITUUT | AMPHIA ZIEKENHUIS | ATRIUM MEDISCH CENTRUM | ORBIS MEDISCH CENTRUM | CATHARINA ZIEKENHUIS | ST. JANS GASTHUIS | MAASTRICHT UNIVERSITAIR MEDISCH CENTRUM I TWEE STEDEN ZIEKENHUIS I REONALD WESTERDIJK 

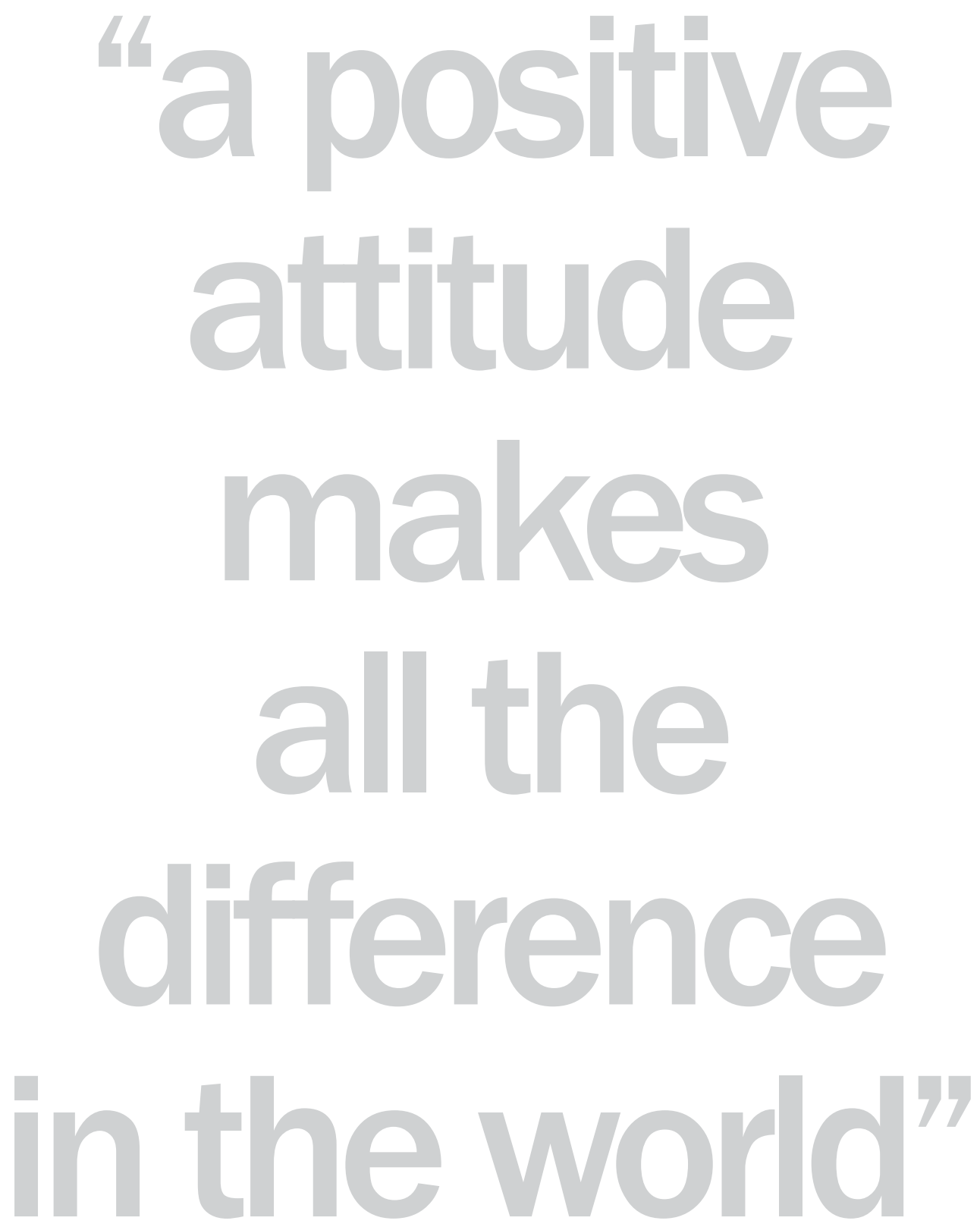

-Unknown artist at MoMA New York 


\section{About the author}

Merel Kimman was born on June $23^{\text {rd }}, 1980$ in Den Haag, the Netherlands. The first years of her secondary school were spent at Reed's school Cobham in England, after which she continued and completed her VWO studies at the Adelbert College in Wassenaar, the Netherlands. She started the Master of Health Sciences at Maastricht University in 1998, and graduated in 2002 after conducting a year of research at the Queensland University of Technology, Australia. Between 2003 and 2005 she worked as a health promotion coordinator at the GGD Oostelijk Zuid-Limburg. In 2005 she applied for the ZonMw funded project 'Improving the quality and efficiency of followup after curative treatment for breast cancer' at Maastro Clinic and Maastricht University. In 2010 she completed her PhD thesis. Merel is currently working as a health economist at the Clinical Trials Centre of the University of Sydney. 
